Proc. Indian Acad. Sci. (Chem. Sci.), Vol. 90, Number 3, June 1981, pp. 153-214. (C) Printed in India.

\title{
Photoacoustic spectroscopy of solids and surfaces $\dagger$
}

\author{
P GANGULY and C N R RAO*
}

Solid State and Structural Chemistry Unit, Indian Institute of Science, Bangalore 560012 , India

MS received 27 June 1981

\begin{abstract}
After briefly reviewing the theory and instrumentation, results from a variety of experiments carried out by the authors on the photoacoustic spectroscopy of solids and surfaces by employing an indigenous spectrometer are discussed in the light of the recent literature. Some of the important findings discussed are, phase angle spectroscopy, anomalous behaviour of monolayers, unusual frequency dependence in small cell volumes, spectra of a variety of solids including amorphous arsenic chalcogenides, photoacoustic detection of phase transitions and determination of surface areas and surface acidities of oxides. Recent developments such as piezoelectric photoacoustic spectroscopy, depth profiling and subsurface imaging are also presented.
\end{abstract}

Keywords. Photoacoustic spectroscopy; optoacoustic spectroscopy; photoacoustic effect.

\section{Intraduction}

Since the late seventies, a new spectroscopic technique called photoacoustic spectro. scopy has come into the forefront. This novel technique has attracted considerable attention because of the ease with which optical spectra of substances normally difficult to examine by conventional spectroscopic methods can be obtained. The technique is of immense value for the study of materials which are opaque, polycrystalline or non-crystalline; it can be equally exploited to study biological systems and surfaces of solids. The technique has been applied to investigate fluorescent yields, depth profiles, phase transitions and so on. Photoacoustic spectroscopy is based on the photoacoustic effect originally discovered

† Contribution No. 124 from the Solid State and Structural Chemistry Unit.

The authors were invited to write this article to commemorate the centenary of the discovery of the spectrophone by Alexander Graham Bell in 1881. The article presents the findings of the authorsin the last few years employing an indigenous spectrometer along with a brief review of this important area.

General Editor Indian Academy of Sciences.

- To whom all correspondence should be sent.

P. (A)-1 
by Alexander Graham Bell in 1881. A bundred years later, today, it would be most desirable to survey and assess the potentials of this technique for the study of solids and surfaces. Although the basic design of a modern photoacoustic spectrometer has the same ingredients as the original spectrophone of Bell, we are now in a much better position to understand the phenomenon and exploit it for various applications. In this article, we have discussed the results of various types of investigations carried out by us in this laboratory employing an indigenous spectrometer in addition to briefly reviewing the theory, instrumentation and recent literature.

\section{Evolution of the modern photoacoustic spectrometer}

Alexander Graham Bell discovered that a large number of materials when taken in the form of thin disks 'emitted sounds " when exposed to the action of a rapidly interrupted beam of sunlight. In a letter to one Mr Tainter dated 2 nd November 1880 , Bell wrote that he has 'devised a method of producing sounds...from substances that cannot be obtained in the shape of thin diaphragms or in tubular form '. In a paper entitled 'Upon the Production of Sound by Radiant Energy' Bell (1881) went on to prove that 'sonorousness, under the influence of intermittent light, is a property common to all matter'. It is instructive to examine the method used by Bell to detect the acoustic signal. "The substance to be tested was to be placed in the interior of a transparent vessel, made of some material which (like glass) is transparent to light, but practically opaque to sound. Under such circumstances the light could get in, but the sound produced by the vibration of the substance could not get out. The audible effects could be studied by placing the ear in communication with the interior of the vessel by means of a hearing tube.' In the letter to Tainter, Bell had noted that he 'got splendid effects from crystals of bichromate of patash, crystals of sulphate of copper and from tobacco smoke. A whole cigar placed in the test tube produced a very loud sound. I could not hear anything from plain water, but when the water was discoloured with ink a feeble sound was heard'.

Bell describes his spectrophone thus: "The eye-piece of a spectroscope is removed and sensitive substances are placed in the focal point of the instrument behind an opaque diaphragm containing a slit. These substances are put in communication with the ear by means of a hearing tube' as shown in figure 1. 'Suppose we smoke the interior of our spectrophone receiver, and fill the cavity with peroxide of nitrogen gas. We have then a combination that gives us good sounds in all parts of the spectrum (visible and invisible) except the ultra-violet. Now pass a rapidly interrupted beam of light through some substance whose absorption spectrum is to be investigated and bands of sound and silence are observed upon exploring the spectrum corresponding to the absorption bands. Of course, the ear cannot compete with the eye in the examination of the visible part of the spectrum but in the invisible part beyond the red, where the eye is useless, the ear is invaluable.'

The quotations above highlight the principles of modern day photoacoustic spectroscopy as well. The main points to note are the use of intermittent light, an acoustic transducer such as the ear, and absorption of light by the material. 


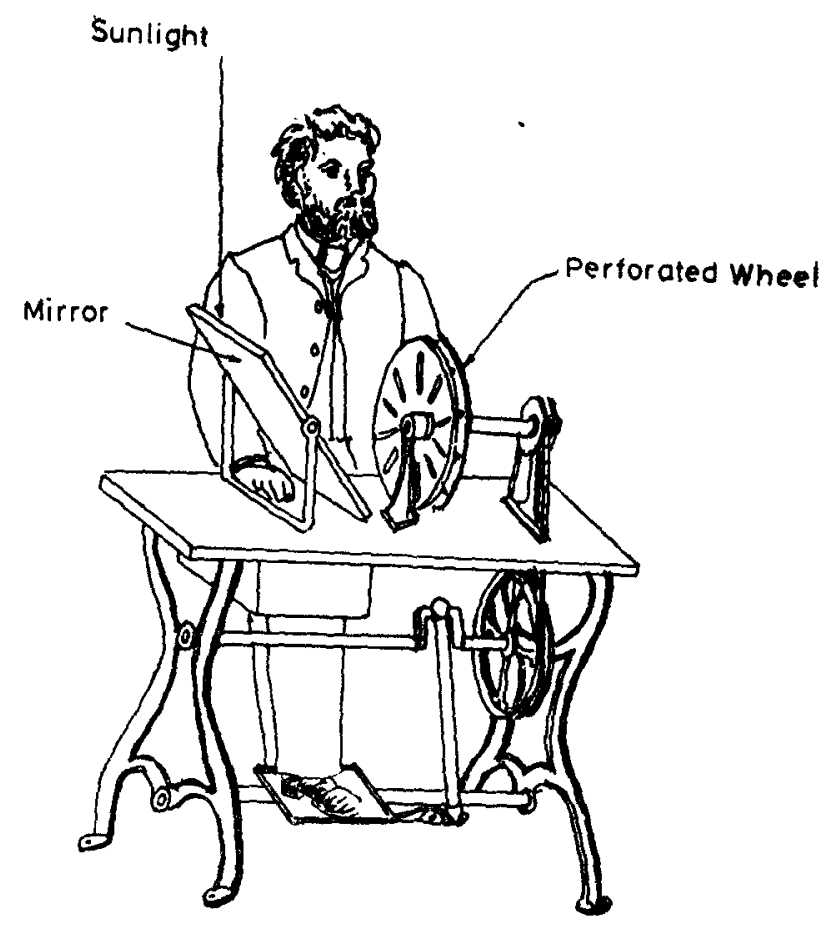

(a)

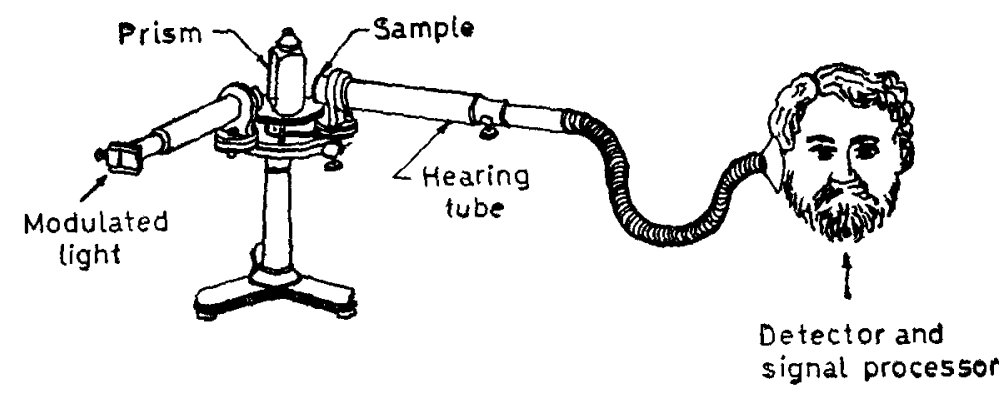

(b)

Figure 1. Bell's spectrophone : (a) chopper (b) spectrophone. (from Bell 1881).

We see that preparation of the material is not critical (so that Bell could use a whole cigar) and that the technique can be used where photodetection is not possible.

Viengerov $(1938,1940)$ used the photoacoustic effect for the measurement of gas concentrations using an acoustically resonant sample cell and recorded the frst absorption spectrum using a prism monochromator and a modulated radiation from a carbon arc. Gorelik (1946) suggested the use of the photoacoustic effect for the determination of energy transfer rates between vibrational and translational degrees of freedom of gas molecules. Slobodskaya (1948) made actual deactivation rate measurements. The photoacoustic effect was extended to microwave frequencies by Hershberger et al (1946). 
The recent revival of the 'spectrophone' as a tool to the study of solids and surfaces owes much to the contributions of Rosencwaig $(1973,1976,1978 \mathrm{~b})$ although the earliest effort to introduce the technique in a systematic manner is perhaps due to Harshbarger and Robin (1973). Prior to the efforts of Harshbarger and Robin, vibrational relaxation rates were examined employing the infrared spectrophone by Kaiser (1959) and Read (1967). Kreuzer and Patel (1971, 1972) were able to detect very low concentrations of contaminants in a gas using a tuneable infrared laser and a microphone transducer. Photoacoustic effect was employed to determine absorption coefficients of thin films by Kerr (1973) and Parker (1973). Kerr called his instrument the alphaphone.

The modern nams for Bell's spectrophone is optoacoustic spectrometer (OAS) or photoacoustic spectrometer (PAS); we have used the latter name in this article. The block-diagram of a modern photoacoustic spectrophotometer is shown in figure 2. Light from a high intensity source such as a high pressure Xe lamp or a tungsten-halogen lamp is passed through a monochromator. The light is then modulated by using a mechanical chopper or by modulating the power of the lamp electronically. The modulation may be done before or after the light passes through the monochromator. The modulated monochromatic light is then allowed to impinge on the sample in a cell. Absorption of light causes heating under certain conditions which in turn causes pressure fluctuation in the ambient gas. This pressure fluctuation results in an audio signal being generated at the same frequency as the chopping frequency. The acoustic signal is then picked up by a suitable transducer. A lock-in amplifier is used to eliminate the noise due to other sources and the intensity of the signal is obtained as a voltage which is plotted on a recorder (or stored in a multi channel analyser) for various wavelengths. Figure 3 compares the microphone signal of a carbon black sample with the calibrated radiometer power spectrum of a $4000 \mathrm{~W}$ Xe lamp. Within the calibrated range of the radiometer, the two spectra are identical. In order to obtain the spectrum of a sample, it is necessary therefore to divide the output of the microphone at various wavelengths by the corresponding power spectrum.

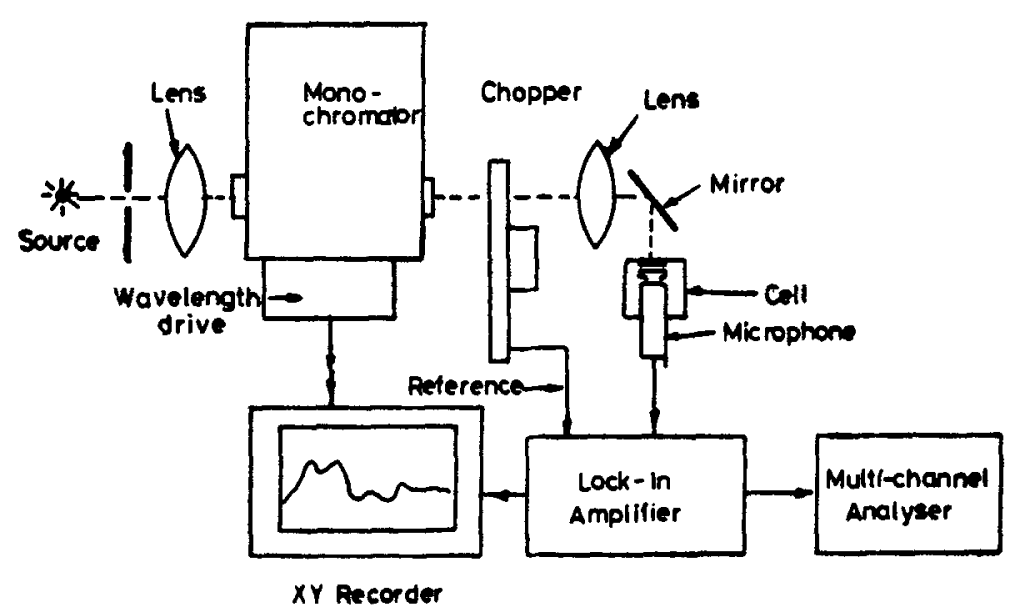

Flgare 2. Block diagram of a modern single-beam photoacoustic spectrometer. 


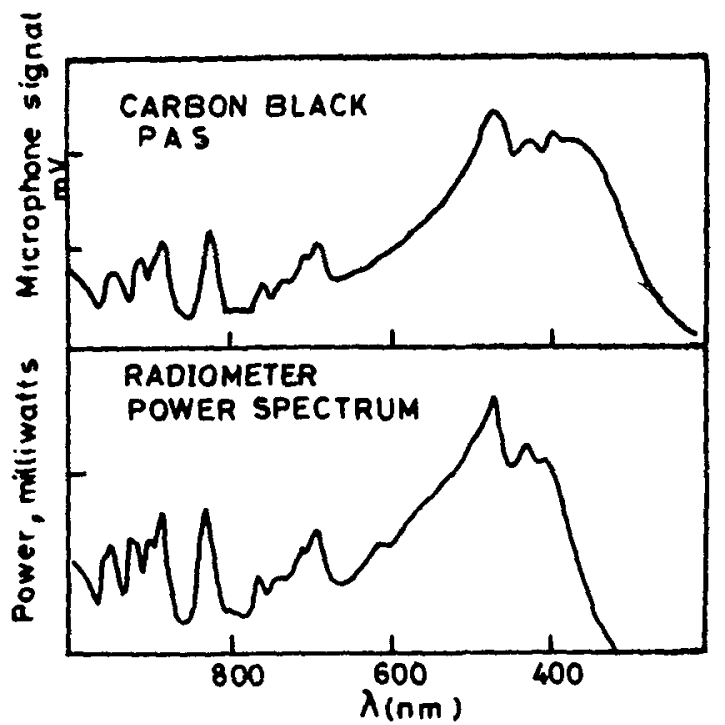

Figure 3. Comparison of the carbon black photoacoustic spectrum and the signal from a calibrated radiometer (From Harshbarger and Robin, 1973).

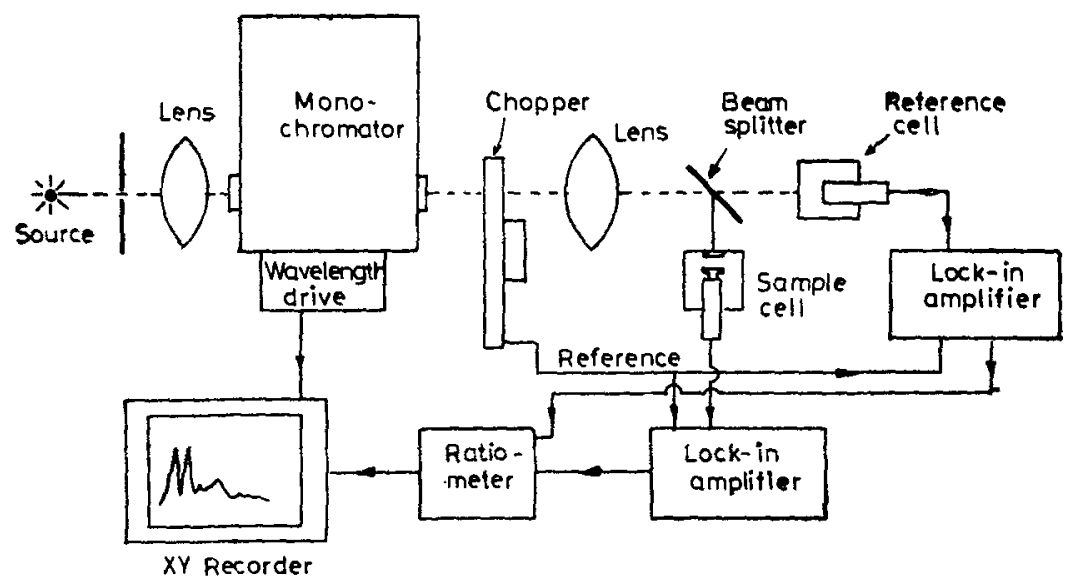

Figure 4. Block diagram of a double-beam photoacoustic spectrometer.

In a single beam spectrometer, this is done by recording the power spectrum of the lamp and the microphone output of the sample separately.

In a double beam spectrometer (figure 4), the monochromatized modulated beam is split into two. One of the beams falls on a thermopile or another PAS cell containing carbon black (which acts as a black body absorber) while the second beam falls on the sample. The output from the sample cell is divided by that from the reference cell by a ratiometer, and the normalized spectrum is recorded directly on a recorder. Piezoelectric detection of pressure waves generated within a sample has also been used to measure photoacoustic signals. 


\section{The photoacoustic effect}

Bell (1881) had considered several mechanisms for the photoacoustic effect. For example, he had noted that the effect was most from loose spongy material and had considered the role of physically adsorbed gases. Bell, however, preferred the view that the sound was due to mechanical vibrations of the solid itself. The conclusive evidence according to Bell was that the sound could be heard a foot away from the point on which the modulated light was focussed. The modern theory of the photoacoustic effect is based on theories for the flow of heat that is intermittently produced. Basically, the effect is identified as a calorimetric effect and thermal diffusion is associated with the flow of heat from the bulk to the surface. The models developed are simple and the mathematical equations derived are exact within the framework of the model. However, all the models are macroscopic in nature and a microscopic explanation of the processes involved have so far not been attempted. The most successful and popular theory is that due to Rosencwaig and Gersho (1976).

Rosencwaig and Gersho (henceforth referred to as RG) developed an exact equation for the magnitude and phase of the photoacoustic signal as a function of the optical, thermal, and geometrical properties of the sample, the cell and the gas within the cell. These authors considered a simple cylindrical photoacoustic cell (figure 5) of diameter $D$, and used the following parameters in their analysis : $D=$ sample diameter in the form of a disk; $l=$ sample length; $l_{g}=$ length of the gas phase which is assumed to be much smaller than the wavelength of the acoustic signal; $l_{b}=$ length of backing material which is in intimate contact with the sample; $\mathbf{x}=$ distance in the cell with the sample surface taken as origin; $\mathbf{t}=$ time; $I_{0}=$ incident photon power $\left(\mathrm{W} / \mathrm{cm}^{2}\right) ; \omega=$ chopping frequency $(\mathrm{rad} /$ sec); $\beta=$ optical absorption coefficient $\left(\mathrm{cm}^{-1}\right) ; \mathbf{k}_{i}=$ thermal conductivity of the material $i$ ( $i=s, g, b$, for solid, gas and backing material, respectively) (cal $/ \mathrm{cm}$ sec deg) $\rho_{i}=$ density of material $i\left(\mathrm{~g} / \mathrm{cm}^{3}\right) ; C_{i}=$ specific heat of material (cal $/ \mathrm{g}$ $\operatorname{deg}) ; a_{i}=k_{i} / \rho_{i} C_{i}$, thermal diffusivity of material $\left(\mathrm{cm}^{2} / \mathrm{sec}\right) ; a_{i}=\left(\omega / 2 \alpha_{i}\right)^{1 / 2}$, thermal diffusion coefficient of material $i\left(\mathrm{~cm}^{-1}\right)$; and $\mu_{i}=l / a_{i}$, the thermal diffusion length of the material $i$ which measures the distance at which the temperature is $1 / \mathrm{e}$ times the maximum source value.

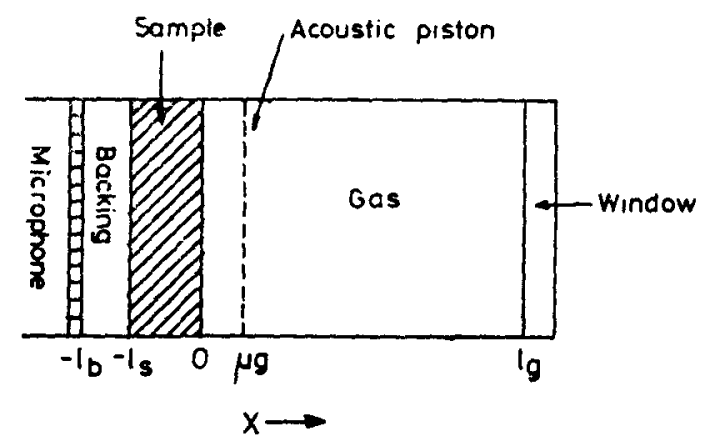

Figure 5. Cylindrical photoacoustic cell model employed in Rosencwaig-Gersho theory (1976). The origin of the $x$ coordinate is the illuminated surface of the sample. 
The intensity $I$ of the light incident on the surface was taken to be sinusoidally related to the chopping frequency and the initial intensity $I_{0}$ as

$$
I=\left(\frac{1}{2}\right) I_{0}(1+\cos \omega t)
$$

Assuming the heat density in the surface or in the solid to be related to the light absorbed, the heat density in the solid using Beer's law formalism becomes,

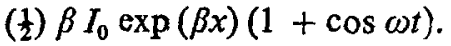

The next step in the RG approach is to set up the thermal difiusion equation in one dimension

$$
\frac{\partial^{2} \phi}{\partial x^{2}}=\frac{1}{a_{i}} \frac{\partial \phi}{\partial t}-A \exp (\beta x)[1+\exp (j \omega t)]
$$

where $\phi$ is the temperature, $j=\sqrt{-1}$ and $A=\beta I_{0} \eta / 2 k_{i} ; \eta$ is the efficiency of conversion of the absorbed light to heat by non-radiative de-excitation processes at the wavelength $\lambda$. The second term in (2) is therefore absent for the gas and backing material which are assumed to be optically transparent. The value of $x$ for which the appropriate thermal diffusion equations are valid in the gas, sample or backing material is obtained from figure 5 .

The solution of (2) is obtained after the appropriate boundary conditions are applied. These boundary conditions must satisfy the requirement of temperature and heat flux continuity at the boundaries of the sample (i.e. at $x=0$ and $x=-1$ ) as well as the boundaries of the cell walls which is assumed to be at ambient temperature. Of interest is the variation in the amplitude of the temperature, $\phi(x, t)$, within the cell as a function of position and time. The timedependent spatial temperature distribution within the cell has a dc and an ac component as well as an exponentially growing component. Since only the ac part is of importance for the production of the photoacoustic signal, the actual physical temperature variation is obtained by taking the real part of the ac component of the solution of the thermal diffusion equation:

$$
T_{a c}(x, t)=\exp \left(-a_{g} x\right)\left[\theta_{1} \cos \left(\omega t-a_{g} x\right)-\theta_{2} \sin \left(\omega t-a_{\nu} x\right)\right] .
$$

Here $\theta_{1}$ and $\theta_{2}$ are the real and imaginary parts of $\theta$ which is the ac component of the complex amplitude of the temperature at the sample-gas boundary; $\theta_{1}$ and $\theta_{2}$ therefore determine the in-phase and quadrature component of the periodic temperature variation. The time-dependent temperature variation is shown in figure 6 as a function of the distance from the sample surface in the gas phase. The temperature variation is seen to attenuate rapidly to zero at a distance of $2 \pi \mu_{\rho}$. A boundary layer of thickness $2 \pi \mu_{g}$ from the surface may then be defined which is capable of responding thermally. The gas within this layer expands and contracts periodically and acts as an acoustic piston on the rest of the gas column.

In order to determine the displacement $\delta x(t)$ of the boundary layer, it is necessary to know the average temperature $\bar{\phi}(t)$, within the boundary layer. This turns out to be

$$
\begin{aligned}
\bar{\phi}(t) & =\left(1 / 2 \pi \mu_{g}\right) \int_{0}^{2 \pi \mu_{g}} \phi_{a c}(x, t) d x \\
& =(1 / 2 \sqrt{2} \pi) \theta \exp [j(\omega t-\pi / 4)] .
\end{aligned}
$$




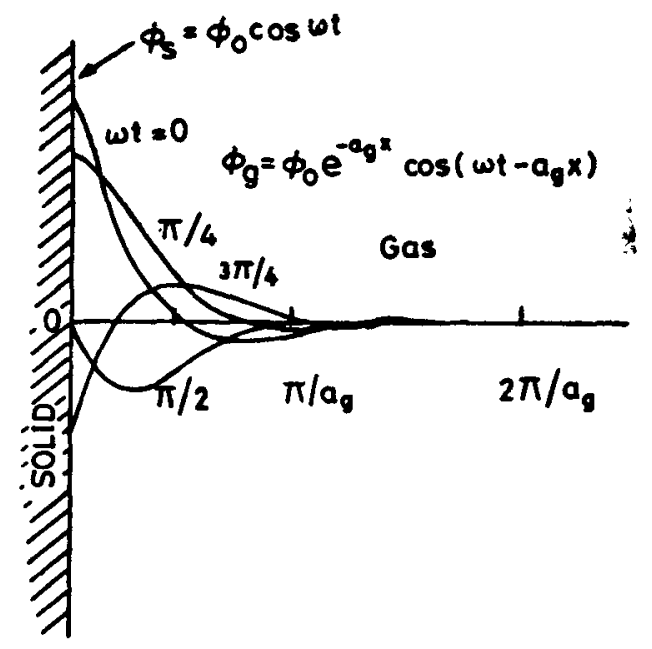

Figure 6. Time dependent temperature variation in the gas phase as a function of distance from the sample surface.

From the ideal gas law, therefore

$$
\begin{array}{ll} 
& \delta x(t) / \bar{\phi}(t)=2 \pi \mu_{g} / T_{0} \\
\text { or } \quad & \delta x(t)=\left(\theta \mu_{o} / \sqrt{2} T_{0}\right) \exp [j(\omega t-\pi / 4)],
\end{array}
$$

where $T_{0}$ is the sum of the ambient tomperature and the dc component of the temperature at the solid surface.

The acoustic pressure in the cell due to the displacement of the acoustic gas piston is obtained from the adiabatic gas law $P V^{\gamma}=$ constant, where $\gamma$ is the ratio of the specific heats. The incremental pressure $\delta P(t)$ is, therefore, given by,

$$
\begin{aligned}
\delta P(t)=\frac{\gamma P_{0}}{V_{0}} d V & =\frac{\gamma P_{0}}{l_{0}} \delta x(t) \\
& =\frac{\gamma P_{0} \theta}{\sqrt{2} T_{0} l_{0} a_{0}} \exp [j(\omega t-\pi / 4)] \\
& =Q \exp [j(\omega t-\pi / 4)] .
\end{aligned}
$$

If we write, $Q=Q_{1}+j Q_{2}=q \exp (-i \psi)$, where $Q_{1}$ and $Q_{2}$ are the real and imaginary parts of $Q$ while $q$ and $\psi$ are the magnitude and phase of $Q$. The actual pressure variation $\triangle P(t)$ is given by the real part of $\delta P(t)$ as

$$
\Delta P(t)=q \cos (\omega t-\psi-\pi / 4) \text {. }
$$

Noting that $Q=\gamma P_{0} \theta /\left(\sqrt{2} l_{0} a_{0} T_{0}\right), \mathrm{RG}$ obtain the following expression for $\theta$ :

$$
\begin{aligned}
\theta= & \frac{\beta I_{0}}{2 k_{a}\left(\beta^{2}-\sigma_{a}^{2}\right)} \\
& \times\left\{\frac{(r-1)(b+1) \exp \left(\sigma_{l} l\right)-(r+1)(b-1) \exp \left(-\sigma_{s} l\right)+2(b-r) \exp (-\beta l)}{(g+1)(b+1) \exp \left(\sigma_{a} l\right)-(g-1)(b-1) \exp \left(-\sigma_{l} l\right)}\right\} .
\end{aligned}
$$


Here

$$
b=k_{b} a_{b} / k_{2} a_{s}, g=k_{p} a_{o} / k_{2} a_{p}, r=(1-j) \beta / 2 a_{1} \text { and } \sigma_{i}=(1+j) a .
$$

Since $k_{g}<k_{g}$, while $a_{g}$ is usually of the same order of magnitude as $a_{s}, g \leqslant 1^{\circ}$ Also, from the definitions of the terms $a_{1}$ and $a_{4}, b \simeq\left(k_{3} / k_{3}\right)^{1 / 2}$ when the $\rho$ 's and $C$ 's are not very different. We see from the above equations that the expression for $\Delta P(t)$ is complicated. In order to obtain physical insight, some special cases need to be considered.

We shall define an optical absorption length $\mu_{\beta}=1 / \beta(\mathrm{cm})$ and consider several cases related to the various relative values of $\mu_{\beta}, \mu_{8}$ and the length of the sample 1. When $\beta$ is small, the value of $\exp (-\beta l) \simeq 1-\beta l$; when $\beta$ is very high, $\exp (-\beta l) \simeq 0$. Similarly, when $\mu_{3} \gg 1, \exp \left( \pm \sigma_{3} l\right) \simeq 1$; when $\mu_{k}<l$, $\exp \left(-\sigma_{s} l\right) \simeq 0$. Similar approximations may be made for $r$. The magnitude of the photoacoustic signal for optically transparent and optically opaque samples are shown for various cases in figure 7. We see from figure 7 that even if the sample is optically opaque, it may be PAS transparent (PA signal being proportional to $\beta$ ) when $\mu_{a}<\mu_{\beta}$ (case $f$ of figure 7).

The exact form of $R G$ theory was used by Rosencwaig (1978a) to study the variation in the magnitude and phase of the photoacoustic signal with the chopping frequency $\omega$. For purposes of universality, a normalized length $L$ was used. For transparent absorbing samples, $L=l / \mu_{\text {r }}$ and for opaque samples, $L=l / \mu_{2} \beta$. For low thermally conducting solids, typical values of $k_{2}\left(\sim 10^{-3} \mathrm{cal} / \mathrm{cm} \mathrm{sec} \mathrm{deg}\right)$ $p_{e}\left(\sim 2 \mathrm{~g} / \mathrm{cm}^{3}\right)$ and $C_{.}(\sim 0.2 \mathrm{cal} / \mathrm{g} \mathrm{deg})$ were employed to generate theoretical curves using eq. (7) for a sample whose length was assumed to be $50 \mu$ for three different values of $b\left(\approx k_{b}^{1 / y} / k_{a}^{1 / 2}\right)=0 \cdot 1,1 \cdot 0$, and 10 and for three different values of $\mu_{\beta}(=1 / \beta)=10 l, l$, and $0 \cdot 1 l$. The theoretical results were compared with the experimental intensity from a $50 \mu$ layer of $\mathrm{GaP}$ at $546 \mathrm{~nm}, 522 \mathrm{~nm}$ and $468 \mathrm{~nm}$ at which wavelengths, $\mu_{\beta}$ for GaP corresponds roughly to the values given above, behaving as a transparent, absorbing and opaque sample, respectively. The results appear to confirm the predictions of the theoretical model fairly well. The magniand of $q$ varies rapidly with the normalized length $L$. The dependence is $\omega^{-1}$ 60. low frequency regions where $L<l$ and is $\omega^{-3 / 2}$ when $L>l$ as expected from the theory (see also figure 7).

Armodt et al (1977) made a systematic study of the dependence of the phase and amplitude of the photoacoustic signal on cell dimensions. A carbon black coating was me on the face of a cylindrical piston in a cylindrical cell. The optical window and the microphone were placed adjacent to each other on one side of the cell, opposite to the carbon black coating. The distance between the carbon black coating and the optical window could be varied by using a micrometer crow to drive the piston. The amplitude of the pressure-changes for constant licht absorption depends on the physical size of the sample cell through the diffu. cion processes and generally decreases with increasing cell size. A normalized loneth $X\left(=\mu_{o} / L\right.$, where $L$ is the length of the gas phase) and a normalized ampliand $q_{0}\left(=\omega^{1 / 2} q\right.$ as the amplitude is related to $\left.\mu_{a}\right)$ was defined to fit the results into a universal curve (figure 8). Although the fit is good, there is a residual fregrency dependince and the amplitude is larger than the theoretical value for hife chopping frequencies or smaller thermal diffusion lengths of the sample. Murpiy and Aamodt (1977a) have shown that the response of a conducting 


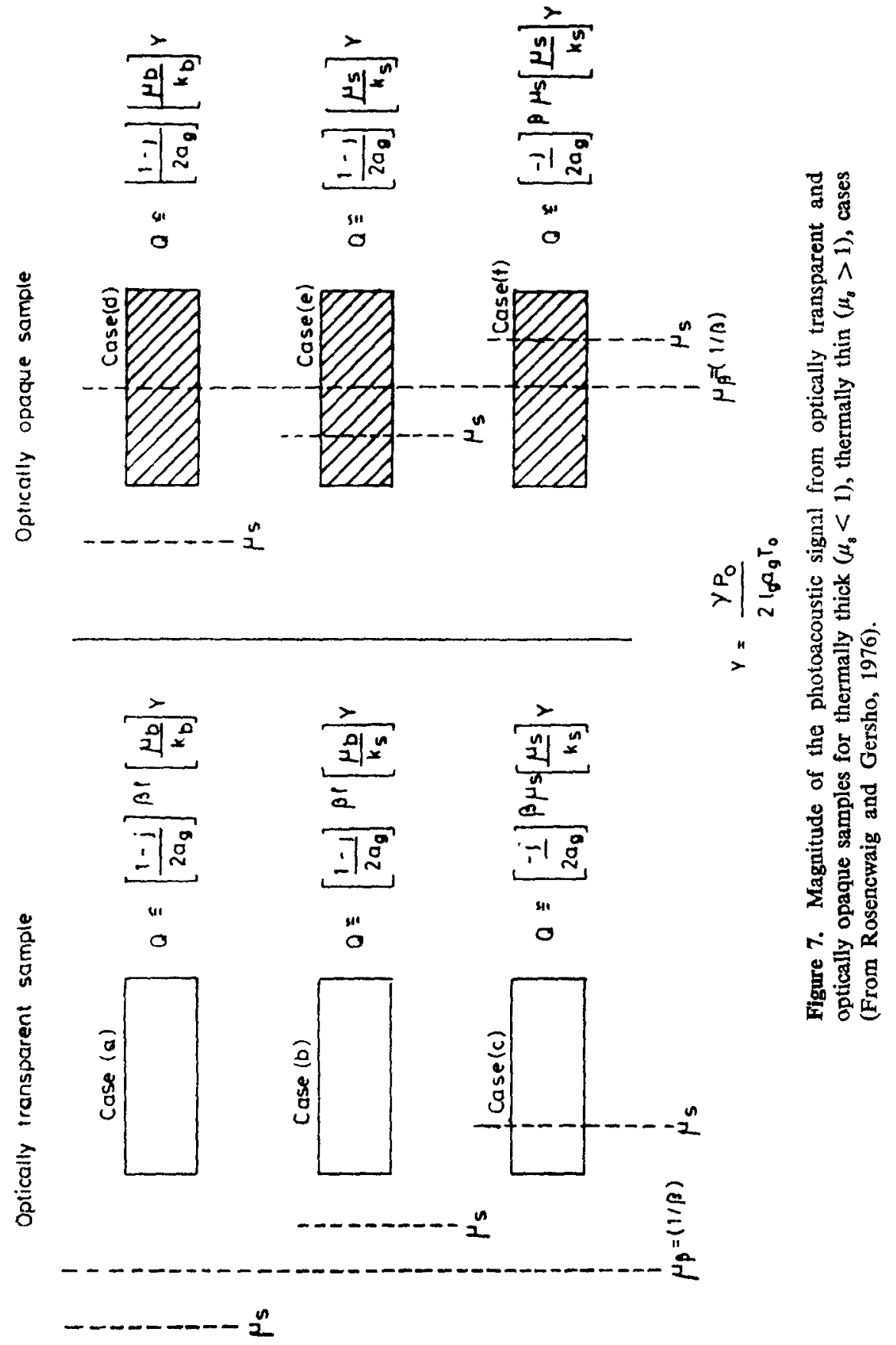




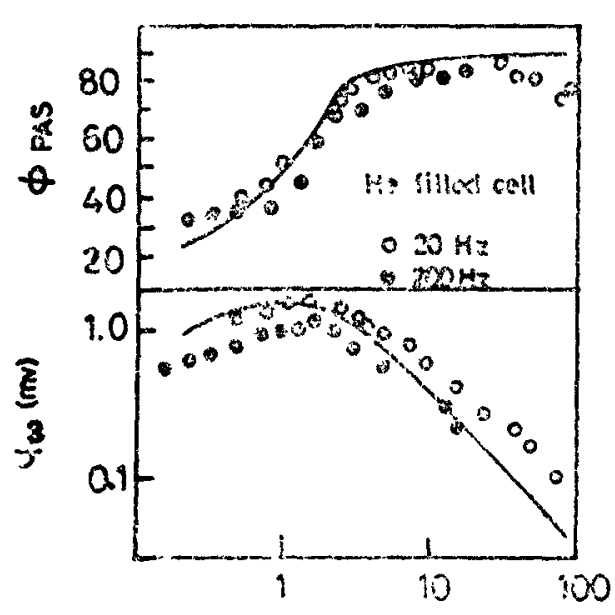

$x=\sqrt{\omega / 2 a g}(1 \mathrm{~cm} / 2.56)$

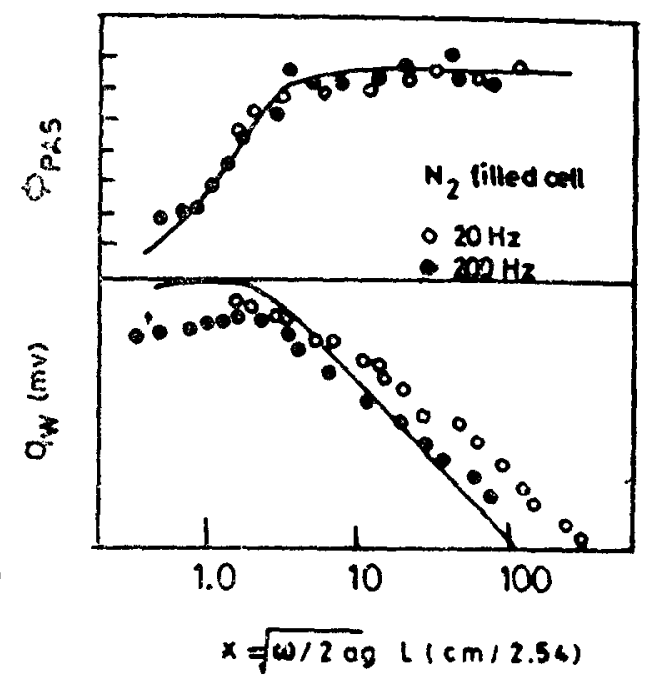

Figure 8. Normalised length $X\left(=\mu_{g} / l_{g}\right)$ vs normalised PA amplitude $q_{\omega}\left(=\omega^{1 / 2} q\right)$ for an helium filled cell and a nitrogen cell (From Aamodt et al, 1977).

platinum black surface is quantitatively the same for both optical and electrical modulated heating.

RG theory is based on a one-dimensional model and the effect of three dimensional heat flow in a real cell may be quite different. McDonald (1980) finds that after a rigorous treatment of the thermal flux problem in three dimensions (assuming that the highly damped thermal waves do not reach the sides of the cell), the thermal flux to the gas is precisely the same as that given by the onedimensional model even if the sample, gas, window or backing is thermally thin in the axial (beam) direction.

Cesar et al (1979) have taken into account the finite surface thermal resistance of the solid in examining the photoacoustic effect. In the RG theory is an assumption of temperature continuity at the surface and gas interface. Cesar et al have noted that such an approximation is valid only for bodies in intimate contact such as soldered joints. In all other cases, the heat flow is proportional to the temperature difference between the two surfaces. The proportionality constant depends on the heat-loss mechanism and is called the coefficient of heat transfer $H$ which is related to the surface thermal resistance $R$ as $R=1 / H$. For nonvanishing $R$, the temperature continuity condition is, therefore, not valid. The case of zero thermal resistance means that the heat t-ansfer between the two media is instantaneous. Employing appropriate boundary conditions, Cesar et al find that for optically opaque and thermally thick solids $Q$, as defined by the RG theory becomes [see equation (6)]

$$
Q=\frac{-j}{2 \pi^{3 / 2}} \frac{\gamma \rho_{0} I_{0} H_{s}}{2 \sqrt{2} \frac{a_{s}^{1 / 2} a_{g}}{l_{g}} T_{0}} \frac{k_{s} k_{g} \omega^{3 / 2}}{}
$$

This result differs from the corresponding one in RG theory through the $\omega^{-1}$ dependence in the latter as well as the dependence on $k_{g}$. For optically opaque and thermally thin solids, however, the frequency dependence is $\omega^{-1}$ just as in 
RG theory and depends on $\left(\alpha_{\theta} / k_{g}\right)$ as in the case of thermally thick solids while RG theory predicts a $\left(a_{3} a_{0}\right)^{1 / 2} / k_{b}$ dependence.

Since thermally thin solids are those in which the thermal diffusion time is smaller than the chopping period, the solid-gas heat transfer may be taken to be instantaneous. However, for thermally thick solids, the heat transfer is not instantaneous. Cesar et al (1979) have chosen a Ge sample from the thermal properties of which they find that above $55 \mathrm{~Hz}$, it should be thermally thick and below $55 \mathrm{~Hz}$, it should be thermally thin. Operating in the wavelength region where the sample is optically opaque, they find that the ratio of the signals from the helium and air-filled cells is the same at the highest and lowest chopping frequencies employed $(30$ and $1530 \mathrm{~Hz}$ ) with the ratio being $1 \cdot 3$. This is the ratio predicted by Cesar et al whereas in $R G$ theory this should be equal to $2 \cdot 7$. They also find that for low chopping frequencies $(25 \mathrm{~Hz})$, when the sample is thermally thin, the signal is independent of the backing material contrary to the prediction of RG theory. Crowley et al (1980) have, however, found that PA signals from opaque thermally thin solids such as $\mathrm{Ni}, \mathrm{Al}, \mathrm{S} \mathrm{C}$, have an $\omega^{-3 / 2}$ dependence when they have a gas backing.

The surface heat resistance argument is a valid one. However, Quimby and Yen (1979b) have shown that the surface heat conductance $H_{8}$ is primarily determined by molecular collisions with the surface; at atmospheric pressures and room temperature, it is much larger than that deter mined by radiative heat transfer. The thermal contact resistance is negligible for most photoacoustically generated signals.

In $R G$ theory, the effect of mechanical vibrations of the sample has been neglected in the calculation of the PAS signal. McDonald and Wetsel (1978) have allowed for acoustic waves in the sample as well as in the gas phase, by solving coupled equations for pressure $P$ and temperature, $\tau$. The coupled equations used are :

$$
\begin{aligned}
& \nabla^{2} p-\frac{\rho_{0}}{\bar{B}} \frac{\partial^{2} p}{\partial t^{2}}=-\rho_{0} \beta_{T} \frac{\partial^{2} \tau}{\partial t^{2}} \\
& \kappa \nabla^{2} \tau-\frac{\kappa}{a} \frac{\partial \tau}{\partial t}+S=-T \beta_{T} \frac{\partial p}{\partial t}
\end{aligned}
$$

where $\rho_{0}$ and $T_{0}$ are the ambient density and the temperature respectively; $B$ is the isothermal bulk modulus, $\beta_{T}$ the coefficient of thermal expansion, $K$ the thermal conductivity, a the thermal diffusivity and $S$ (present in the sample only) the thermal energy source due to optical absorption. These authors have developed a composite piston model to take into account the mechanical vibrations of the sample. The composite piston is the total effect due to the thermal acoustic piston of RG theory and the mechanical piston. The results of the calculation of McDonald and Wetsel (1978) are compared with that of the RG theory in figure 9. We see that the acoustic amplitude increases considerably from RG theory at high chopping frequencies and low absorption coefficients; at $\omega=100$ $\mathrm{Hz}$, the mechanical piston effect gives $20 \%$ of the signal for $\beta=10 \mathrm{~cm}^{-1}$ and $50 \%$ of the signal for $\beta=1 \mathrm{~cm}^{-1}$. Such low absorption coefficients are possible in liquids. By getting the best fit of the experimental curve with the theoretical curve using the optical absorption coefficient as a variable, McDonald and Wetsel have been able to obtain quantitative values of absorption coefficients of liquids. 


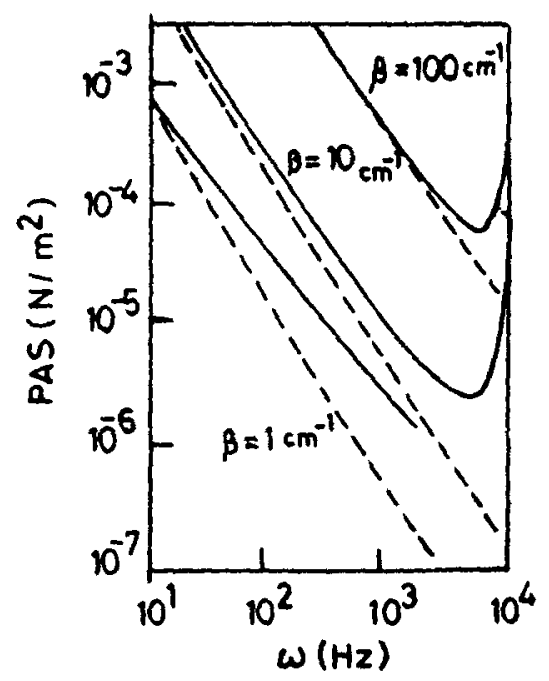

Figure 9. Theoretical values of PA signal intensity from the theory of McDonald and Wetsel (1978) shown by solid lines for three values of $\beta$. Resonance is seen at high frequencies for the coupled equation treatment. Dashed line is that obtained from RG theory. Sample is a dye solution in water.

The mechanical piston effect is considered to be negligibly small for solids as the total heat generated by the non-radiative de-excitation if taken up totally by the solid for thermal expansion can give only $1 \%$ of the observed signals due to the mechanical piston effect. However, some recent results suggest that there may be cases where the mechanical expansion has to be considered.

Adams et al (1976) have studied the effect of using different filler gases and have found that the PA signal amplitude increases as the heat capacity of the gas increases. They have also studied the effect of particle size and have noted that the PA signal amplitude increases as the particle size decreases.

The RG theory shows that the PA signal is proportional to the ratio of specific heats $\gamma$ of the gas used to couple the pressure variation to the microphone detector. In general, helium-filled cells $\left(\gamma_{\mathrm{не}}=1.66\right)$ give a larger PA signal than nitrogenor air-filled cells $\left(\gamma_{N_{2} / 11 r}=1.404\right)$ (Wong 1978; Aamodt et al 1977). Aamodt et al (1977) have predicted the variation of the signal intensity for helium and nitrogen-filled cells and find that under certain conditions (such as small cell volume), helium filled cells give a lower signal intensity. The RG theory predicts an enhancement of the signal on filling a cell with any gas as compared to nitrogen

$$
\mathrm{EF}=\left(\gamma_{0} / \gamma_{\mathrm{N}_{2}}\right)\left(a_{o} / a_{\mathrm{N}_{2}}\right)^{0.50}
$$

Here, EF, the enhancement factor, is the ratio of the signal from the gas-filled cell to the nitrogen-filled cell and $a$ is the thermal diffusivity. Wong (1978) has found that EF from a plastic sheet coated with black paint for a helium-filled cell obeys the relationship,

$$
\mathrm{EF}_{\mathrm{zc}}=(0.90 \pm 0.15)\left(a_{\mathrm{Bo}} / a_{\mathrm{N}_{\mathrm{g}}}\right)^{0.37 \pm 0.03}
$$


compared to the expected value of $(1 \cdot 05 \pm 0 \cdot 10)\left(a_{\mathrm{He}} / a_{\mathrm{N}_{2}}\right)^{0.50}$. This observation of Wong does not find agreement with the predictions made from a more rigorous treatmont by Bennett and Forman (1976) which predicts a $(\gamma /(\gamma-1))$ dependence. Using the empirical relationship for the enbancement factor, EF (empirical $)=0.9\left(a_{p} / \alpha_{\mathbb{N}_{3}}\right)^{0.37}$, Wong defines a ratio, $\mathrm{EF}^{+}=\left(\mathrm{EF}_{\text {exptl }} / \mathrm{EF}_{\text {empirtes }}\right)$, where EF (exptl) is the enhancement factor measured experimentally from activated charcoal. It is found that $\mathrm{EF}^{+}$varies in the order $\mathrm{CO}_{2}>\mathrm{Ar} \geqslant \mathrm{N}_{2} \geqslant \mathrm{Air}>\mathrm{H}_{2}$ $>\mathrm{He}$. This is just the order in which the various gases are physically absorbed by activated charcoal. EF ${ }^{+}$also shows a linear relation with the van der Waals constant of gases. Wong has proposed that the absorbed gas forms a region of high density very close to the solid surface and hence significantly enhances the piessure variation. The amplified pressure variation is, according to Wong, larger than the reduction in thermal diffusivity which accompanies the higher density.

\section{The photoacoustic cell}

The important criteria for the construction of a photoacoustic cell (Rosencwaig 1977) are the following :

(i) The material for the cell should exhibit good attenuation and be of sufficient thickness to form a good acoustic barrier. The thermal mass of the cell walls should be large. Acoustic isolation from the environment is necessary; this can be done by keeping the cell in a sound-proof box mounted on springs for isolation from ambient vibrations in the room as well as from chopper vibrations. The table on which the spectrometer is kept could also be isolated.

(ii) The windows should not only be transparent in the region of interest but should also be good attenuators of sound. Optically transparent fused quartz windows are ideal for the purpose.

(iii) Minimization of scattered light reaching the cell walls and the microphone diaphragm is an important criterion. For this purpose, the geometry of the cell should be such that the microphone is kept away from the beam path and the sample is directly below the window with the window being larger than the sample dimensions so that the scattered light or unabsorbed light is reflected out of the cell. Impurities in the sample holder or in the cell which absorb light should be scrupulously eliminated. The cell walls should, therefore, be highly polished and the sample holder easy to clean or replace. It should also be easy to clean the window of the cell periodically.

(iv) The cell dimensions should be such as to minimize its volume since the PAS signal varies inversely with the cell volume.

(v) Thermoviscous damping is an important parameter (Rosencwaig 1977). The thermoviscous damping coefficient varies as $\omega^{1 / 2}$ and becomes important at high frequencies. The cell should, therefore, have minimum distance between the sample and the window and maximum passageway dimensions between the sample region and the microphone. The dimensions suggested by Rosencwaig is typically 1 to $3 \mathrm{~mm}$.

(vi) In or der to improve the acoustic signal, it is possible to work with Helmholtz resonance cells or work with specially designed cylindrical microphones. Limi- 
tations in these cases are : the frequency response of the microphone is not flat and the cell cannot be used at frequencies other than the resonance frequency.

Typical cell designs are shown in figures 10 and 11 . In most cell designs, the microphone section is connected by a narrow passage to the sample section. One has to worry about resonance effects between volumes connected by a narrow passage (Munroe and Reichard 1978; Fernelius 1979). For this reason, the signal versus frequency response may not comply with the theoretical requirements as discussed earlier for the one-dimensional model. The usual cell geometry is such that the flat electret microphone is mounted perpendicular to the direction of incidence of light. In the one-dimensional model, the thermal wave in the gas phase propagates in the direction directly opposite to that of the incident light. The closest approximation to such a cell design is that by Aamodt et al (1977). These authors have shown that the signal strength is maximum when $\mu_{0}=L$, the length of the gas phase.

Ferrell and Haven (1977) have used a configuration in which the microphone is placed opposite to the incident light direction. The sample is placed on a paper positioned between the window and the microphone. A pinhole in the paper is sufficient to connect the microphone and sample compartments to obtain signals without distortion or diminution.

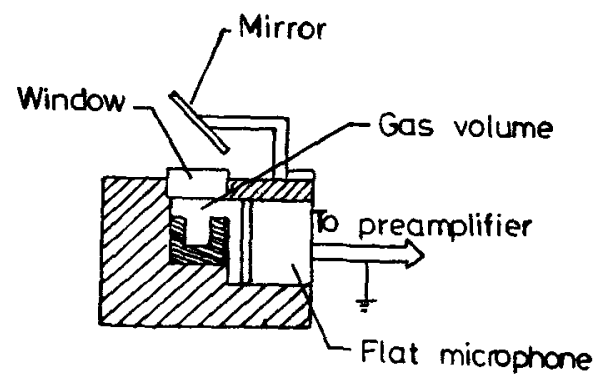

(a)

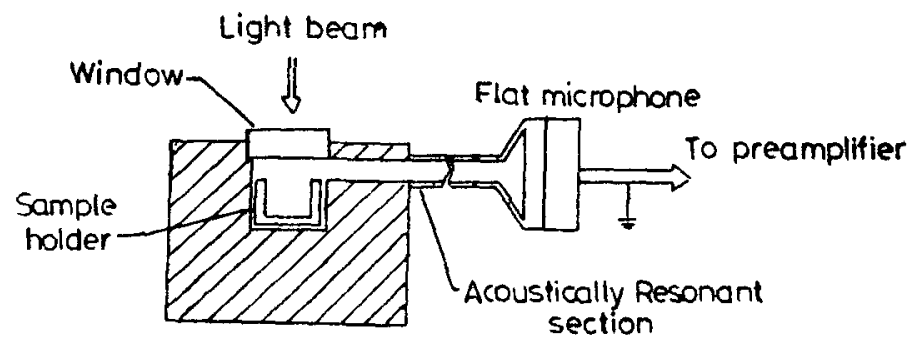

$(b)$

Figure 10. (a) Simple photoacoustic cell with flat microphone (After Rosencwaig,

1977). (b) Helmboltz resonant cell (From Rosencwaig, 1977). 


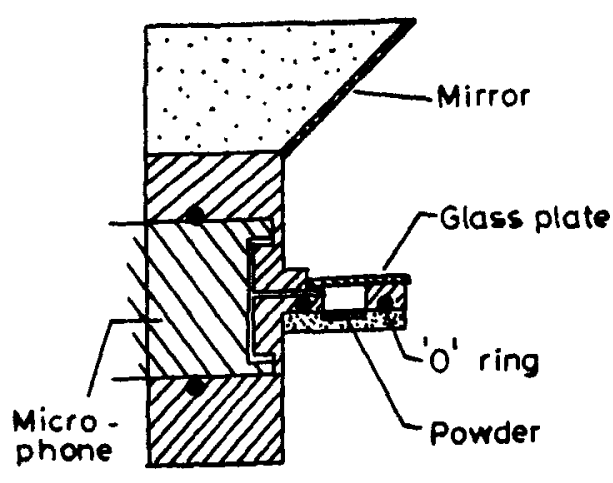

(a)

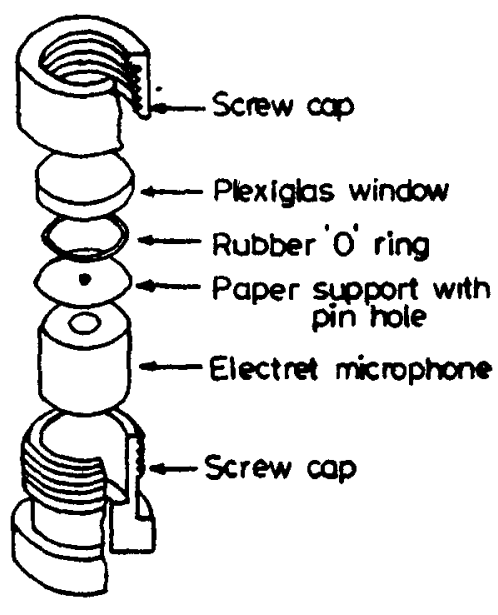

(b)

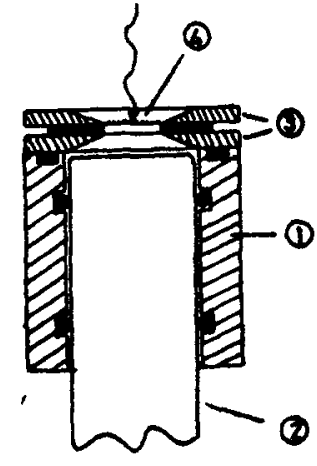

1. Microphone housing,

2. Microphone, 3.Membrane mount 4. Membrane with somple

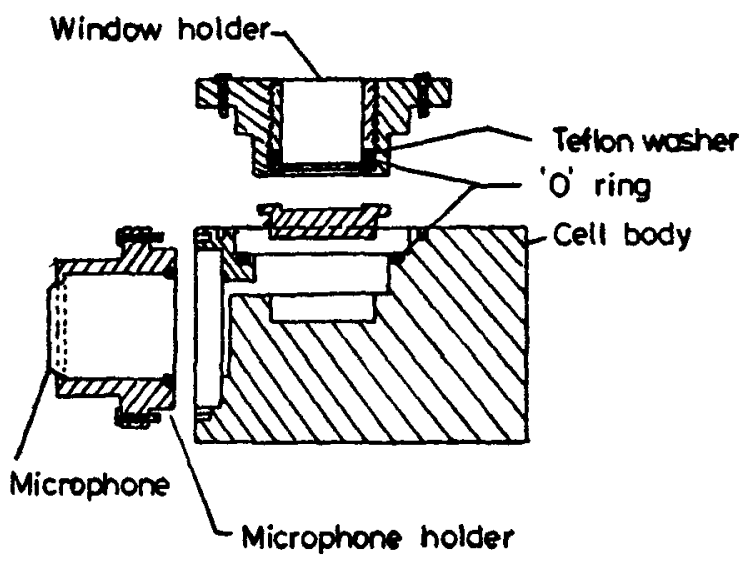

(c)

Figure 11. Typical cell designs (a) from Monahan and Nolle, 1977; (b) from Ferrell and Haven, 1977; (c) open membrane spectrophone after Kanstad and Nordal, 1978 ; (d) after Ducharme et al (1979). 
Ducharme et al (1979) have described cell (figure 10) with a signal-to-noise ratio of 3000 to 1 for a light power of $1 \mathrm{~mW}$ and a time constant of $1 \mathrm{sec}$ at $100 \mathrm{~Hz}$ chopping frequency. They use a cylindrical stainless steel sample holder in the shape of a disc with a depression of $1 \mathrm{~mm}$.

\section{Fabrication and performance of an indigenous photoacoustic spectrometer}

We have fabricated a single-beam photoacoustic spectrometer essentially as in figure 2. For use in the visible region a totally indigenous spectrometer using a $24 \mathrm{~V}$ dc $250 \mathrm{~W}$ tungsten-halogen lamp, an Emco lock-in amplifier, a CEL monochromator, and an indigenous electret microphone would be adequate for the purpose provided the chopping frequency is kept reasonably low $(10 \mathrm{~Hz})$. For such low chopping frequencies the fabrication of a chopper is also fairly easy. For most of our studies we have employed a PAR model 124 A lock-in amplifier, a GR 1962-9601 $\frac{1}{2}$ inch or 1 inch electret microphone with nearly fiat frequency response in the $5 \mathrm{~Hz}$ to $2 \mathrm{kHz}$ frequency range, and a PAR model 195 chopper. The heart of the instrument is of course the cell and the design adopted by us has evolved slowly over a period of time.

The cell design adopted by us is shown in figure 12. This cell features a replaceable cylindrical sample holder which could be made out of Teflon, alumina, stainless steel, aluminium, etc. The sample holder has two notches on opposite sides which connects the space above the sample to the microphone which is positioned below the sample holder which fits tightly into the cylindrical cavity. The sample holder is sufficiently thick so that no light is transmitted through the sample holder to the microphone below. In this configuration, the problem of scattered light affecting the signal as mentioned by McClelland and Kniseley (1976b) is minimized. After the sample holder is mounted in the cell, the top part of the cell containing the optical window is screwed on, the 0-rings making the cell air-tight. We have found that for reproducible results the incorporation of an aluminium spacer in the design helps tremendously as it limits the extent to which the screws can be tightened while fixing the optical window. In principle, the protective grid of the microphone may be removed to increase the intensity.

The sample holder has a cylindrical depression the depth of which may be varied in different sample holders. Typically the sample holder has a depression which is $10 \mathrm{~mm}$ in dia and $1 \mathrm{~mm}$ in depth. The height of the sample holder is typically $3.5 \mathrm{~mm}$ and its diameter is $13.5 \mathrm{~mm}$. The distance $d_{m}$, between the sample window and the top of the sample holder may be varied by changing the height of the sample holder. We have found that the optimum signal is obtained when $d_{w o} \approx \mu_{0}$ in agreement with the results of Aamodt et al (1977). One of the advantages of the cell design adopted by us is the ease of machining and the flexibility of the cell for incorporating changes.

The image of the slit is condensed to $8 \mathrm{~mm}$ in height using a suitable condenser lens assembly and is directed to the surface by a mirror. In order to obtain reproducible intensities we have found that the slit image should be positioned such that the entire image of the slit falls on the sample. Quimby and Yen (1979) have analysed the $3 d$ heat flow and have found that a distance of the order of $\mu$, should be maintained between the sample and the cell walls. The dimensions 

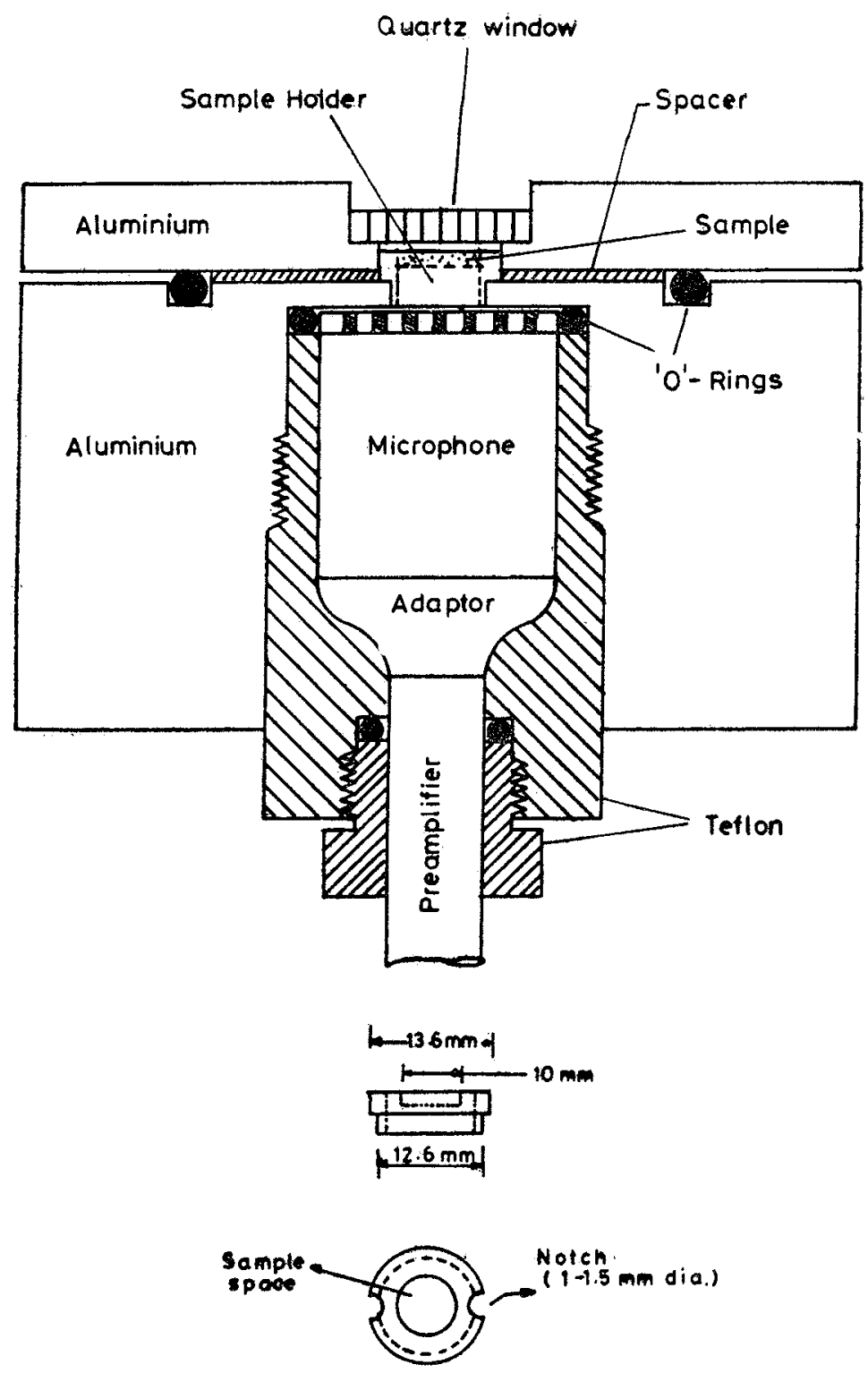

SAMPLE HOLDER

Figure 12. PAS eell employed by the authors.

of our cell and sample holder take this into account for the lowest chopping frequencies $(10 \mathrm{~Hz})$ and the largest slit widths. For higher chopping frequencies and narrow slit widths, large volume decreases the signal intensity; this is overcome by using suitable spacers.

As a test of the performance of our photoacoustic spectrometer we show the normalized spectrum of $\mathrm{Ho}_{2} \mathrm{O}_{3}$ obtained by us (figure 13). The spectrum was taken immediately after the $\mathrm{Ho}_{2} \mathrm{O}_{3}\left(99.9 \%\right.$ purity) was heated at $1000^{\circ} \mathrm{C}$ for $12 \mathrm{hr}$ 


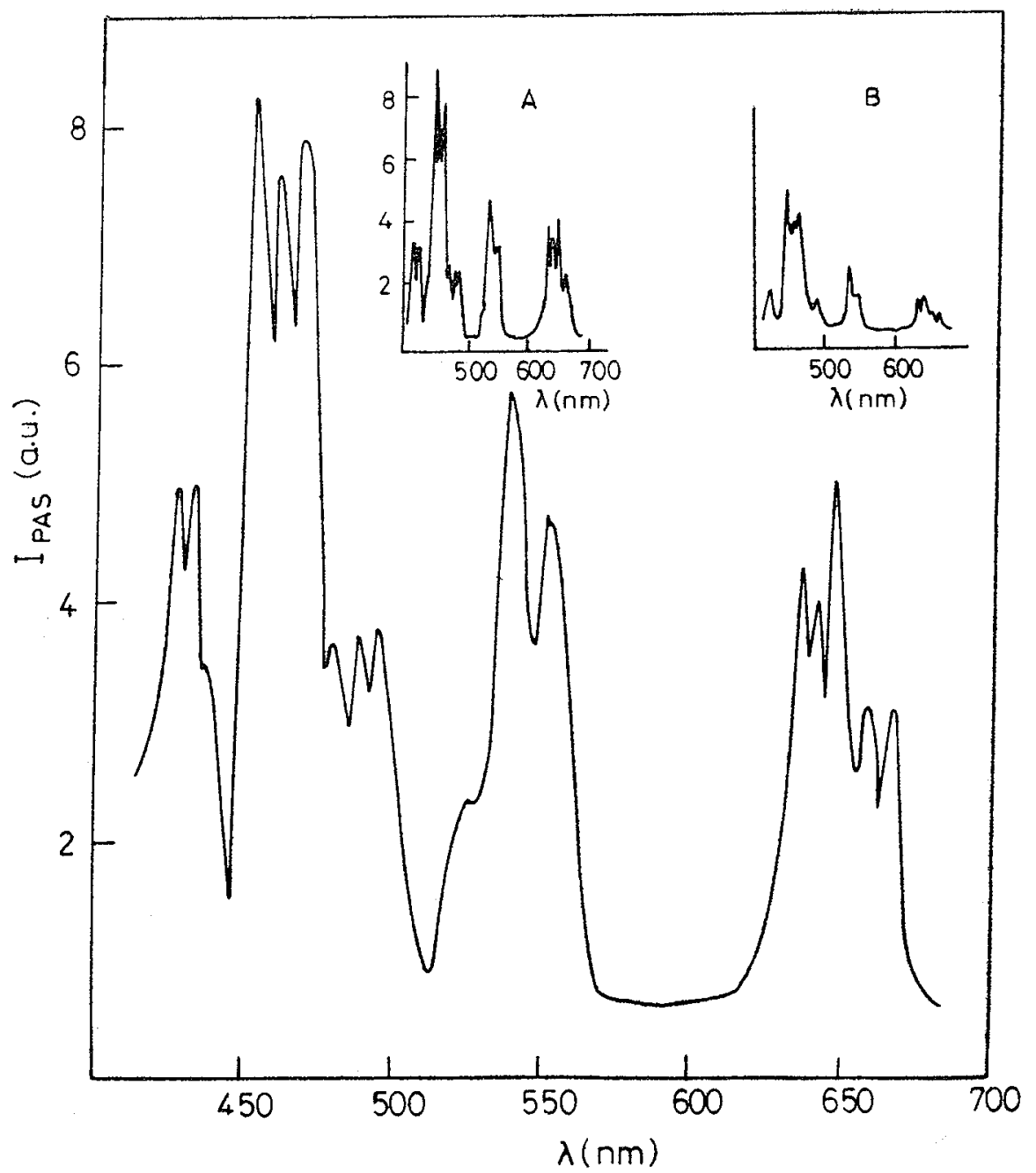

Figure 13. Normalized spectra of $\mathrm{Ho}_{2} \mathrm{O}_{8}, \Delta \lambda=2 \mathrm{~nm}$, Time constant $=3 \mathrm{sec}$, wavelength drive $=10 \mathrm{~nm} / \mathrm{min}$. Inset $A$ from Eaton and Stuart, 1978 ; inset $B$ from Adams et al (1977). Intensity $I_{P A S}$ is in arbitrary units (a.u.).

and cooled to ambient temperature in a desiccator containing $\mathrm{P}_{2} \mathrm{O}_{5}$. The spectrum was run at $10 \mathrm{~nm} / \mathrm{min}$ with a slit width of $0.66 \mathrm{~mm}$ to give a resolution of $2 \mathrm{~nm}$. The spectrum compares favourably with that reported in the literature (Eaton and Stuart 1978; Adams et al 1977). We have observed that the intensities of some of the lines are sensitive to water content.

In order to characterize the cell, one generally examines the frequency dependence of the intensity and phase of the signal from a carbon black sample. Since we used a PAR model 195 chopper, we could obtain signals only for certain fixed frequencies. The phase of the signal is sensitive to the position of the chopper. Thus, when the chopping frequency is changed by changing the chopper wheel, the phase of the signal may also change. We show in figure 14 the $\log I_{\text {ras }} v s \log \omega$ 


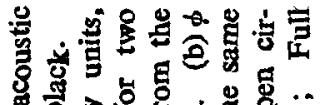

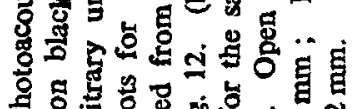

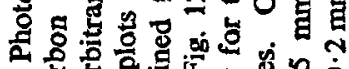

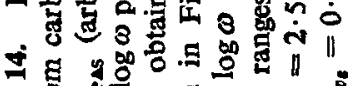

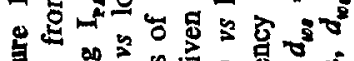

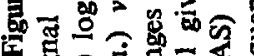

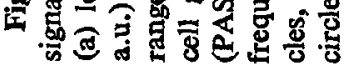
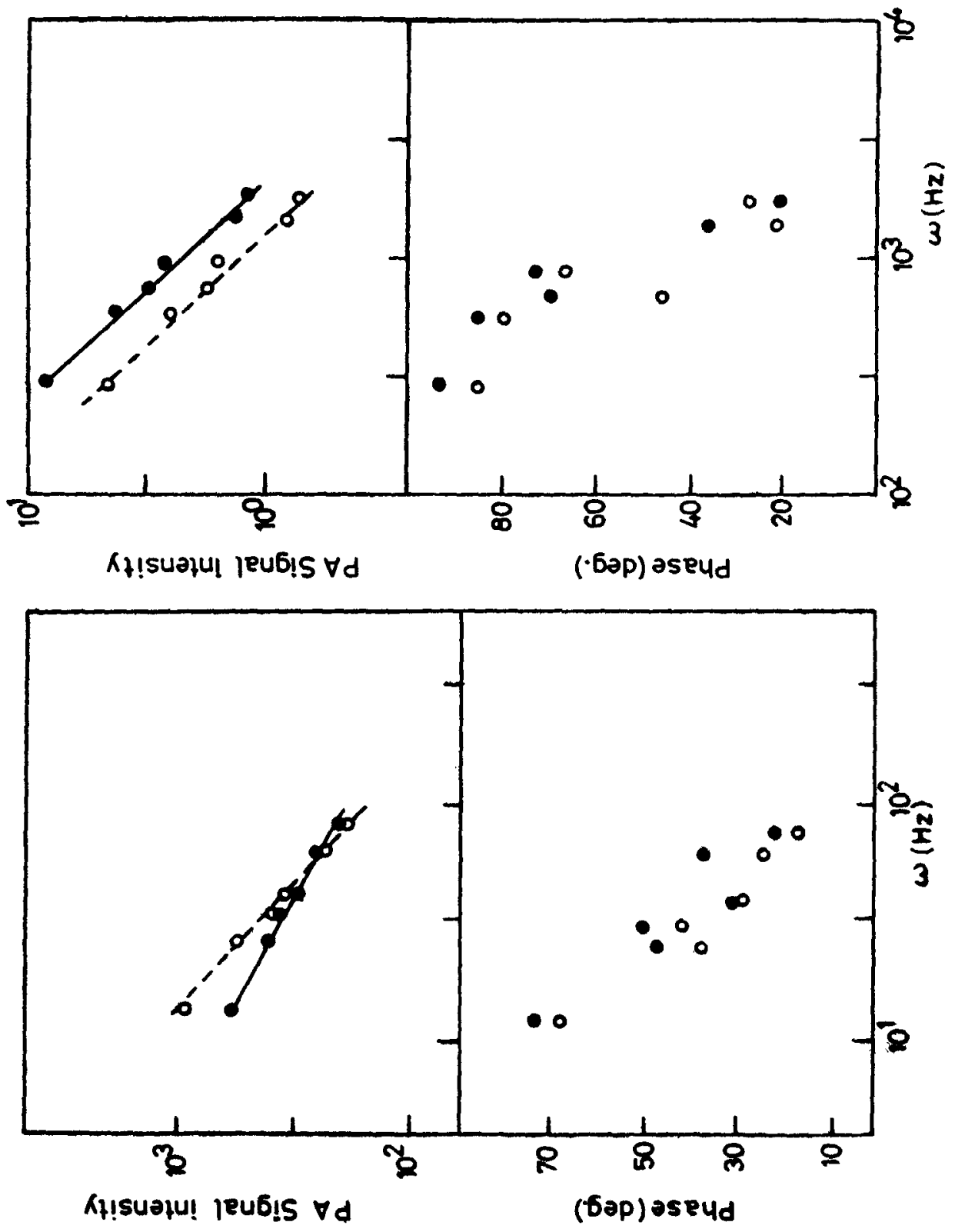
and $\phi$ (PAS) vs $\log \omega$ plots for two sets of chopping frequency ranges corresponding to two different chopper wheels (with 2 and 48 apertures, respectively). The results are shown for two different values of $d_{x s}(2.5 \mathrm{~mm}$ and $0.2 \mathrm{~mm})$. In the high chopping frequency ranges $\left(>250 \mathrm{~Hz}\right.$ ), the $\log I_{\text {rAs }}$ vs $\log \omega$ plots give straight lines for both values of $d_{k,}$ with a slope corresponding to a chopping, frequency dependence of $\omega^{-1}$ as predicted by RG theory. However, at lower chopping frequencies $\left(<100 \mathrm{~Hz}\right.$ ) we obtain a $\omega^{-1}$ dependence only when $d_{\text {wos }}=$ $2.5 \mathrm{~mm}$ whereas when $d_{w o s}=0.2 \mathrm{~mm}$ we obain a $\omega^{-0.50} \pm 0.05$ dependence. This dependence is obtained whenever $d_{\mathrm{st}}<\mu_{0}$ and the new $\omega^{-1 / 2}$ dependence obtained by us could indeed be real. When $d_{u,}<\mu_{0}$ the signal intensity decreases with decreasing $d_{x, .}$. The variation of the phase of the photoacoustic signal shown in figure 14 is somewhat different from that predicted by $R G$ theory.

Murphy and Aamodt (1980) have examined the PAS signal in the time domain after a pulsed excitation and found two waves (an acoustic wave and thermal wave) are generated. The acoustic wave travels with the velocity of sound and its transit time in a PAS cell is therefore determined by the dimensions of the cell and the velocity of sound in the media. The thermal wave is much slower. In longer cells where only the acoustic wave is detected during the observation time, the computed peak PAS response is proportional to input power and inversely proportional to cell length. In shorter cells where both the acoustic and thermal waves are detected during the observation time, the peak response is not proportional to the input energy and the PAS response decreases with decreasing cell length. The thermal wave interferes with the acoustic wave for small cell dimensions. Their treatment does not, however, account for the $\omega^{-1 / 2}$ dependence that is observed by us for small cell dimensions.

The $\omega^{-1}$ dependence in the RG theory has been interpreted by McDonald and Wetsel (1978) as due to two $\omega^{-1 / 2}$ terms. One of these is associated with the thermat diffusion in the solid by which the heat generated at a chromophore inside the bulk diffuse to the surface. This is related to $\mu_{\text {a }}$ which would give a $\omega^{-1 / 2}$ term. The resultant heat from the intermittently heated surface has to diffuse to the gas phase to drive the thermal acoustic piston. This also results on a $\omega^{-1 / 2}$ term due to $\mu_{g}$. As a consequence, we obtain $\omega^{-1 / 2}$ dependence. When $d_{\text {re }} \ll \mu_{\theta}$, the thermal acoustic piston effect is inoperative and we have only a residual effect from surface heating. We are therefore led to believe that the $\omega^{-1 / 2}$ dependence observed by us is likely to be real and not an artifact.

The variable temperature cell fabricated by us (figure 15) has a simple design. Basically the sample chamber extends out of the cell and is surrounded by a hot or cold finger. The heat transfer from the hot or cold finger to the sample is mainly by radiative processes. The signal from the sample chamber is transmitted through a capillary to the microphone cell. The capillary should be a poor conductor and a good attenuator of sound and hence quartz is ideal. For low-temperature work (figure 15a) the sample chamber is made out of metal, the capillary being attached to the metal with the aid of a suitable seal. The O-ring in the sample chamber limits the use of the cell to temperatures less than $200^{\circ} \mathrm{C}$. Figure $15 \mathrm{~b}$ shows a cell that we have used for high temperature phase transition studies. In both these cells a three-way narrow bore stopcock isolates the sample chamber from the microphone chamber. Until the sample chamber is thermally equilibrated with the surroundings, it is kept in contact with the atmosphere with 


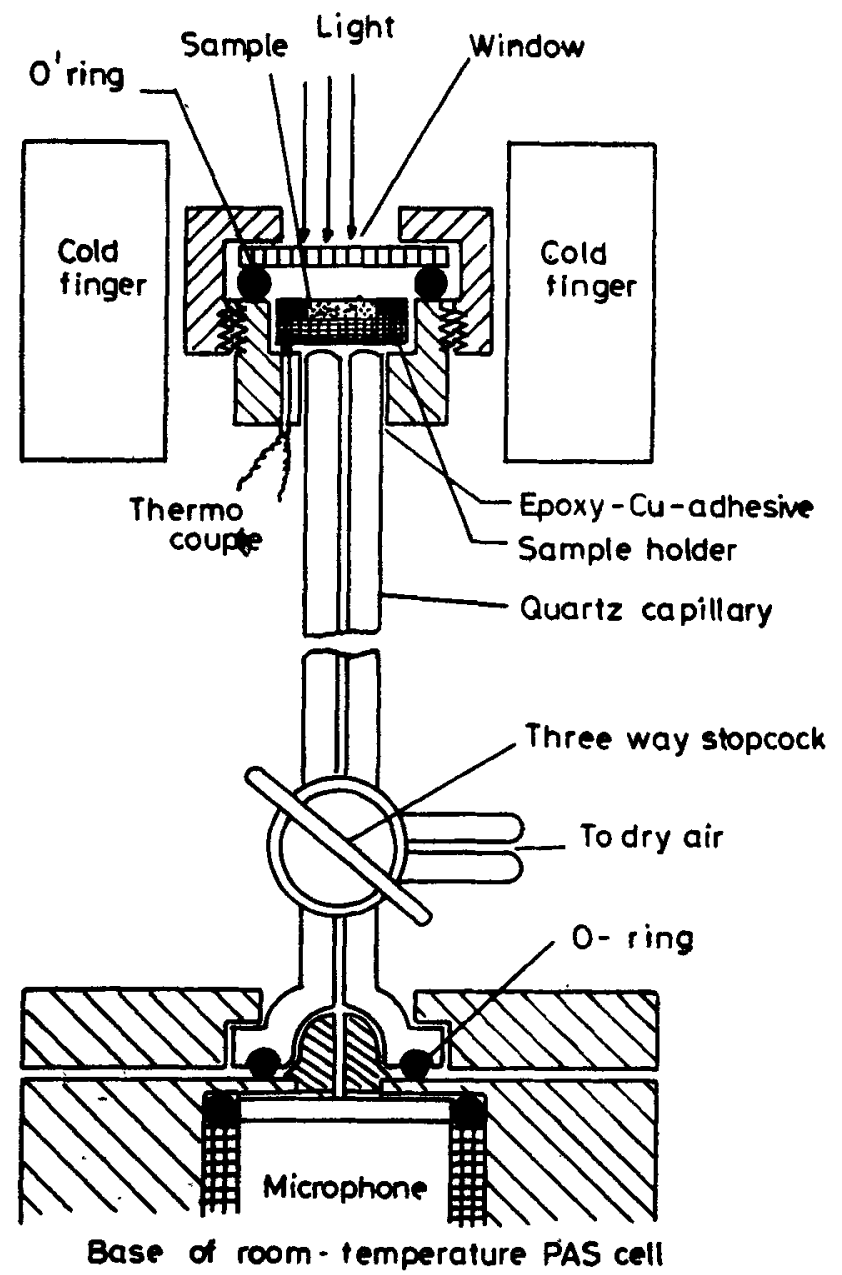

(a)

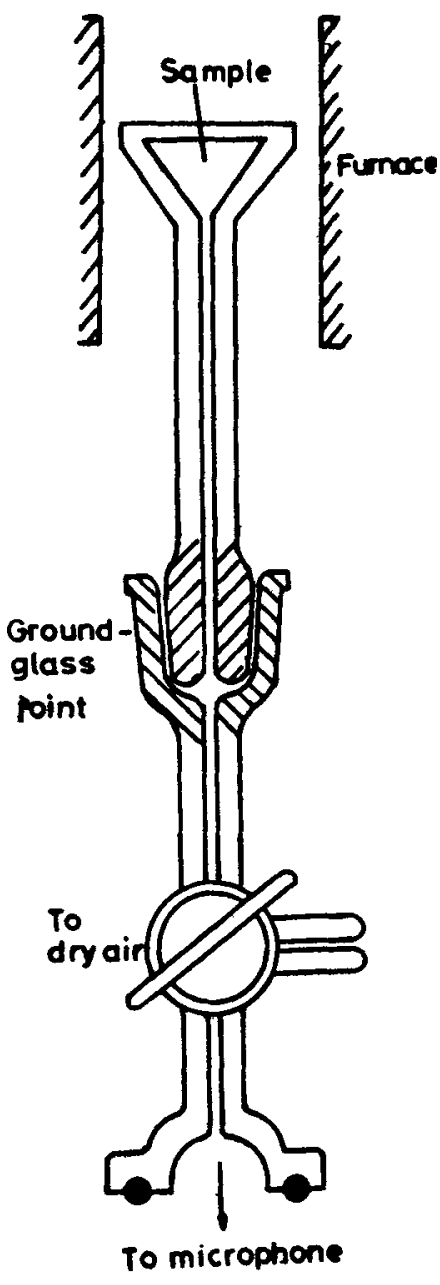

(b)

Figure 15. Variable temperature PAS cell used by the authors. (a) for lowtemperature use ; (b) for phase transition studies at temperatures greater than $200^{\circ} \mathrm{C}$.

suitable traps to prevent the condensation of gases such as $\mathrm{CO}_{2}, \mathrm{H}_{2} \mathrm{O}$, etc. inside the chamber at low temperatures. Once the sample chamber reaches a steady temperature it is connected to the microphone chamber.

In most cases, it is found that while heating a fresh sample the PA signal increases with temperature instead of decreasing as expected from RG theory [see for example equations (5) and (6)]. The signal increases until it reaches a particular temperature after which it starts decreasing. On cooling, however, the maximum is usually not obtained and the PAS signal increases with decreasing temperature. On reheating the PA signal decreases with increasing temperature 
roughly obeying the $1 / T$ law. The maximum obtained on heating a fresh sample is indeed strange and we feel that this is associated with the presence of a large amount of absorbed or condensed phase on the sample.

\section{Phase and intensity in PAS}

It is customary to obtain spectra of samples at a phase angle, $\phi$, where the quadrature component is zero for the maximum in the absorption coefficient. However, for varying absorption coefficients, the $R G$ theory predicts that the phase would change and that quantitative PA spectra may not be obtained. In figure 16, we show the uncorrected PA signal amplitude as a function of wavelength for an amorphous $\mathrm{As}_{20} \mathrm{Se}_{80}$ specimen obtained at $11.6 \mathrm{~Hz}$ chopping frequency for a sample holder in which $d_{w e}$ was $2.0 \mathrm{~mm}$. The signal from a carbon black specimen is also shown. The normalized PAS spectrum is shown in the inset. The $\mathrm{As}_{20} \mathrm{Se}_{\mathbf{8 0}}$ (amorphous) sample shows a band edge at $\sim 600 \mathrm{~nm}$. The phase at which the signal is maximum also changes with the wavelength as shown in the inset of the figure. The uncorrected spectrum obtained when the signal was maximized with respect to the phase at 600 and $800 \mathrm{~nm}\left(\phi=250^{\circ}\right.$ and $229^{\circ}$ respectively) are significantly different as seen from figure 16 where the intensity of the signals were normalized to be the same at $800 \mathrm{~nm}$. The point-by-point recording of the spectrum every $20 \mathrm{~nm}$ at the phase angle $(\phi)$ that gave the maximum in the signal for each wavelength is also shown. We see that the spectrum rocorded at $\phi=250$ 。 corresponds fairly well to the point-by-point signal amplitudes. We also show in the figure the PA signal amplitude when the spectrum was seen at $\phi=319^{\circ}$ and $340^{\circ}$ corresponding to the quadrature component being zero at 800 and $600 \mathrm{~nm}$ respectively. We see that the amplitude of the signal at $600 \mathrm{~nm}$ for $\phi=319^{\circ}$ is quite high while at $340^{\circ}$ the signal is rather low. These results seem to indicate that spectra obtained at a single phase angle may not measure true intensities.

When a chromophore is adsorbed as a monolayer on the surface $\mathbf{R G}$ theory does not predict a change in the phase at various absorption coefficients $\beta$ or as an absorption band is scanned. We find, however, that phase-dependent signals are obtained even from an absorbed monolayer. Phenolphthalein is by itself colourless. When adsorbed on surfaces of some oxides, it develops a red colour. The red colour is possible only from the first layer of phenolphthalein that is adsorbed on the surface. In figure $17 \mathrm{a}$ we show the spectrum of phenolphthalein adsorbed as a monolayer from an ether solution on a $2 \% \mathrm{Na}_{2} \mathrm{O}-\mathrm{Al}_{2} \mathrm{O}_{3}$ catalyst. Curve A shows the normalized spectrum of adsorbed phenolphthalein. There is negligible signal from the region above $700 \mathrm{~nm}$. Even for this monolayer, we get a significant change in the phase as seen from curve $E$ of figure $17 b$. This is quite in contrast with the predictions of RG theory and is unexpected.

In order to understand the phase dependence a little better we introduced an additional signal by introducing (a) a little bit of carbon black into the sample and (b) an external sound at the same frequency (At $71 \cdot 3 \mathrm{~Hz}$ chopping frequency. the chopper noise enters the cell if the cell is not placed in a sound-proof box). The amplitude of the signal in the presence of carbon black shows a shift in the base line as seen from curve $B$ in figure $17 \mathrm{a}$. The curve $F$ in figure $17 \mathrm{~b}$ showe 


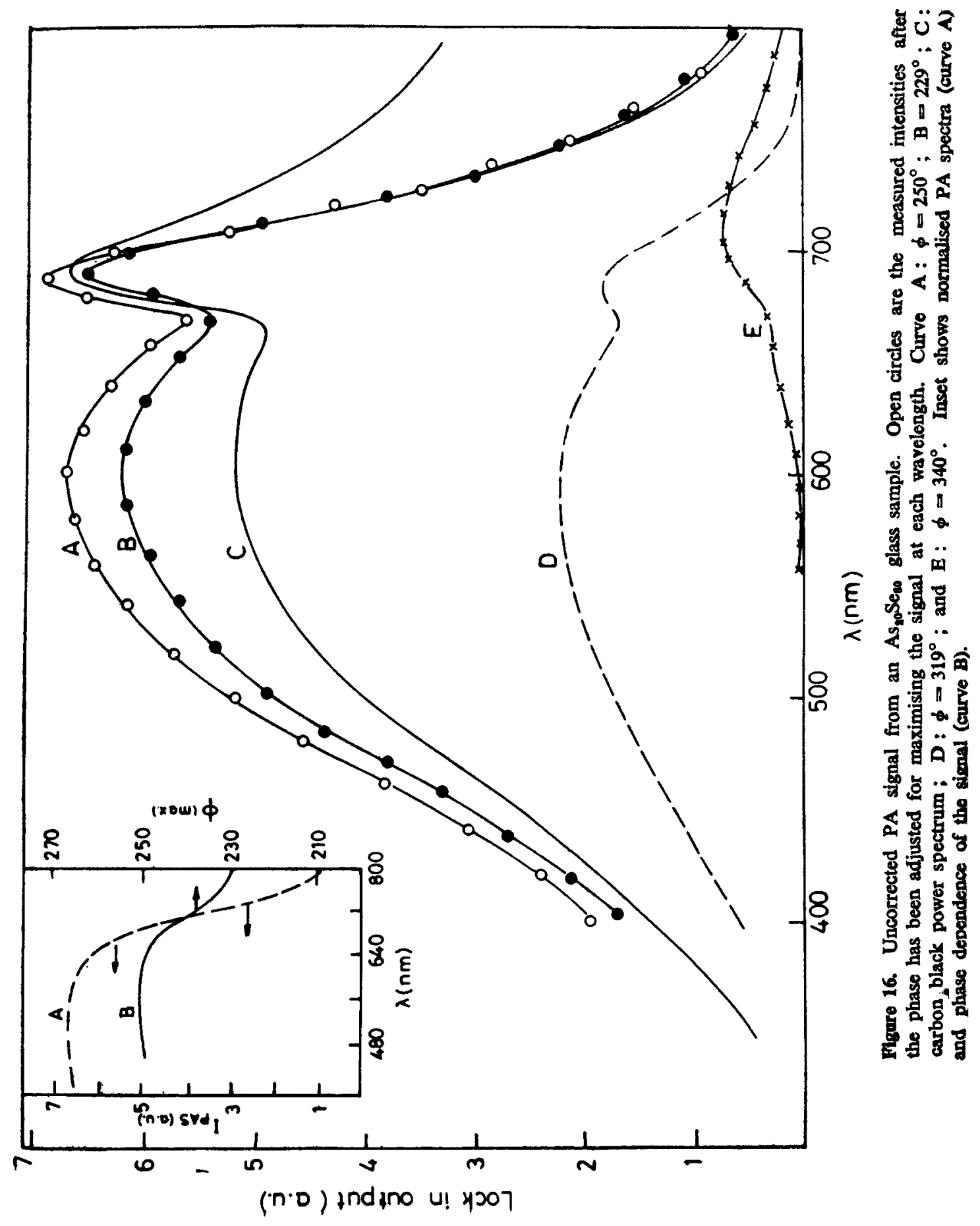



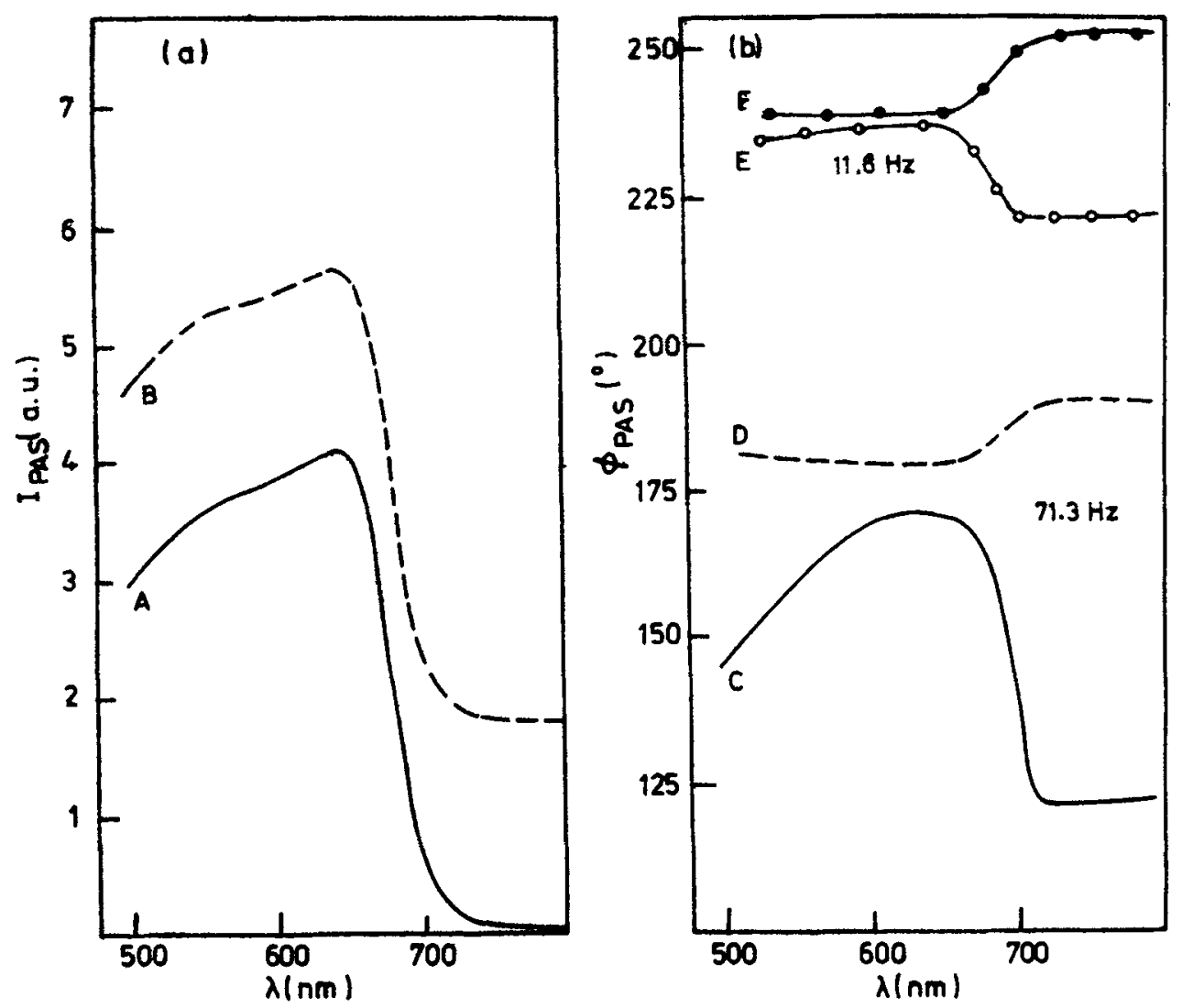

Figure 17. (a) Normalised PA spectrum of phenolphthalein absorbed on a $2 \%$ $\mathrm{Na}, \mathrm{O}-\mathrm{Al}_{2} \mathrm{O}$, catalyst surface from an ether sohution (Curve $\mathrm{A}$ ); Curve $\mathrm{B}$ is the spectrum from the same sample mixed with a very small amount of carbon black; (b) Phase dependentce of the signal. See text for details.

the phase change. The observed phase change suggests that the signals from the earbon black and the phenolphthalein monolayer are generated at different phases. A similar effect is also found when an external sound is introduced (curve $\boldsymbol{C}$ in figure $17 \mathrm{~b}$ ). The phase shift is especially marked at low values of the absorption coefficients. The total amplitude of the signal within the cell due to the external noise in the absence of light was measured to be $5 \%$ of the PAS amplitude of the sample at the absorption maximum. In the presence of carbon black, however, the shift in the phase is much less (curve D).

The phase dependence of the signal from a monolayer may be due to trace amounts of impurities, although all precautions were taken by us to remove any traces of impurities and chopper sound. Especially at the lowest chopping frequency used by us $(11 \cdot 6 \mathrm{~Hz})$, the PA signal is nearly zero with very clean cells and even then shows the phase dependonce is seen.

The phase dependence could also be due to two different signat sources which have different phases. For typical microphones (response $1 \mathrm{mv} / \mu$ bar) and cell dimensions $\left(d_{x,}=2-3 \mathrm{~mm}\right.$, cell volume $\left.1 \mathrm{cc}\right)$, the PA signal is generally of the order of I $\mathrm{mV}$ at $100 \mathrm{~Hz}$. This corresponds to a displacement of $20-30 \mathrm{~A}$ of the acoustic piston 
or a displacement of $10^{14}$ molecules into the ambient volume. For small values of $\beta$ or at high chopping frequencies, the signal is of the order of $0.1-0.01 \mathrm{mV}$. Under these conditions, therefore, besides the thermal acoustic piston effect and the mechanical piston effect (McDonald and Wetsel 1978), effects due to adsorbed gas being displaced to the atmosphere may become important. An empirical relationship between the amplitude of the PAS signals of various gases and their physical absorption on the sample has indeed been suggested by Wong (1978). It may therefore be appropriate to consider what can be termed as the "adsorbed piston" effect in which the periodic heating of the surface results in a periodic displacement of adsorbed gas molecules into the ambient atmosphere. For the pariodic heating of the surface only the thermal diffusivity of the solid is important and we therefore expect a $\omega^{-1 / 2}$ dependence. This is the dependence that we obtain for small values of $d_{\omega o}\left(\ll \mu_{o}\right)$ when the thermal acoustic piston effects are expected to be small. Since the heat reaching the surface would first affect the adsorbed gas, we may also conclude that the "adsorbed piston" effect would be operative before the thermal acoustic piston effect.

According to the RG theory, the thermal gradient at the excited surface causes the heat flow back into the cell. The temperature gradient at the surface caused by the generation of heat dominates and contributes to the photoacoustic signal as predicted by the one-dimensional RG theory. Further into the sample, the heat flow is dominated by the temperature gradient $\mathrm{I}$ adially out fi om the excited region which corresponds to the cross-section of light absorption. This heat does not generally reach the surface within the duty cycle of the PAS experiment and hence would not contribute to the photoacoustic signal.

The photoacoustic signal increases with the absorption coefficient $\beta$, due to two mechanisms : (i) the more complete movement of the initial heat distribution into the thermally active layer $\left(\mu_{\mathrm{s}}\right)$ and (ii) the movement of the distribution closer to the thermal transfer surface $\left(\mu_{\beta}<\mu_{s}\right)$. Saturation effects begin to appear when $\mu_{s}=\mu_{\beta}$ when the rate of diffusion of thermalised energy into the sample becomes nearly the same as the rate of energy input. The increase in the signal when $\mu_{\beta}<\mu_{a}$ is due only to mechanism (ii). When the heat distribution saturates against the thermal transfer surface, the signal becomes independent of $\beta$. In the region from the onset of saturation to full saturation, the photoacoustic signal progressively loses its sensitivity to $\beta$. The photoacoustic spectra in this region would, however, exhibit maxima corresponding to those in the absorption spectra. In the region where the signal is independent of $\beta$, the photoacoustic signal depends only on the sample reflectivity with the reflection peaks coinciding with the minima in the photoacoustic spectra.

In the quantitative determination of absorption spectra by PAS, the major limitation is due to signal saturation in regions of high absorptivity (McClelland and Kniseley 1976a). In terms of RG theory, saturation may be avoided, in principle, by changing the modulation frequency so that $\mu_{0}<\mu_{\beta}$ in the entire region of the spectrum. The limitation of this method is that the thermal diffusion length is propartional to $\omega^{-1 / 2}$ while the signal strength is proportional to $\omega^{-3 / 2}$ McClelland and Kniseley (1976a) have analysed the conditions for overcoming the problems of saturation. Samples whose thickness, $d$, is less than $\mu_{z}$ are expected to have saturation onset at $\beta=2 \pi / d$. Signals will be especially enhanced, therefore, when samples are in thin transparent form. In the case of highly 
absorbing materials which are soluble in solvents without disintegration or dissociation, such as dyes or inorganic complexes, spectra of unimolecular layers of the dye or of thin multi-molecular layers may be obtained easily. It has been shown by Lin and Dudek (1979) that when a large amount of powdered tetraphenylporphin (TPP) is examined, PAS shows only the absorption edge (figure 18). The quality of the spectrum improves gradually on reducing the quantity of TPP and becomes comparable to the optical spectrum in solutions. The extinction coefficient of a strong absorber like TPP $\left(\varepsilon=5.4 \times 10^{5} \mathrm{~m} / \mathrm{L} . \mathrm{cm}\right)$ is so high that

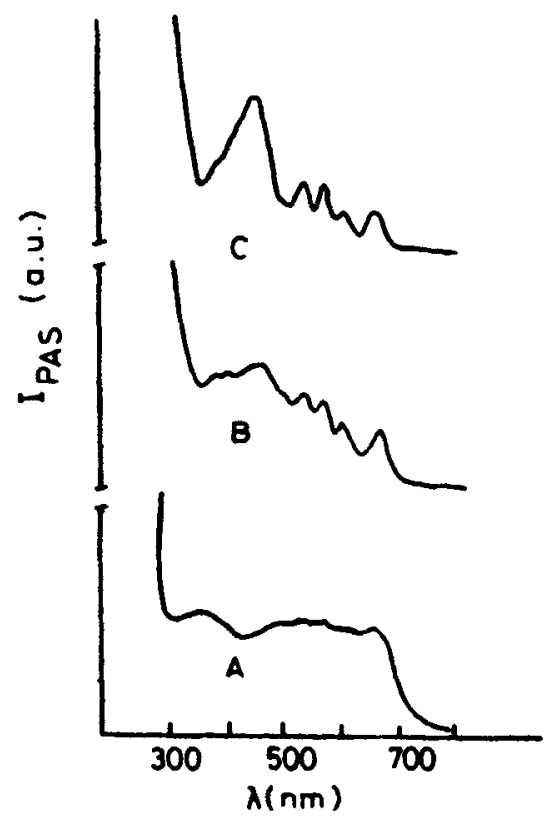

(a)

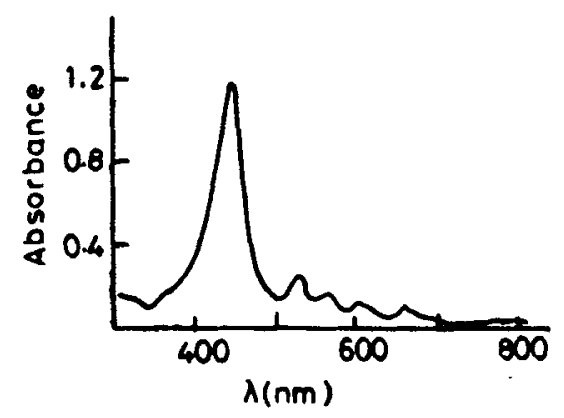

(b)

Figure 18. (a) Normalised spectrum of tetraphenyl porphin TPP : (A), Excess powder; (B), $16.6 \mu \mathrm{g}$ of TPP deposited on a quartz plate ; (C), 3.0 $\mu \mathrm{g}$ of TPP deposited on a quartz plate. (b) Optical absorption spectrum of a smear of TPP on a quartz plate (From Lin and Dudek, 1979). 
only very small quantities are required for saturation. Signal saturation is not observed if large quantities of an inert absorber are mixed with the sample. The dye may be deposited on highly roflecting substracts such as $\mathrm{BaSO}_{4}$ and $\mathrm{MgO}$ or mixed into them by mechanical grinding. We have abtained the spectrum of KTCNQ charge transfer salt which as a solid shows saturation over the whole visible region, even though it has an intense blue colour. When deposited on alumina from a solution in acetonitrile, however, all the features of KTCNQ are readily seen in the spectrum (recorded soon after solvent evaporation) to avoid chemical interaction between adsorbent and adsorbate. Freeman et al (1980) have obtained excellent spectra of $\mathrm{K}_{2} \mathrm{Cr}_{2} \mathrm{O}_{7}$ by mixing it with $\mathrm{MgO}$. There is indeed a similarity between PAS and diffuse reflectance spectra.

Monahan and Nolle (1977) have pointed out that when $\beta$ is small compared to the particle diameter, the optical penetration depth is refractively determined and is insensitive to $\beta$; the intensity of the PAS signal is then similar to that found by diffuse reflectance. The intensity of the PA signal will vary with $\beta$ according to Melamed's (1963) analysis of diffuse reflectance experiments; for high values of $\beta, \mathrm{RG}$ theory will be valid. The absorption coefficient of amorphous $\mathrm{As}_{2} \mathrm{~S}_{3}$ calculated from the relative absorption is given by

$$
\text { Relative absorption }=\frac{\mathrm{As}_{2} \mathrm{~S}_{3} \text { signal }-\mathrm{CaF}_{2} \text { signal }}{\text { charcoal signal }-\mathrm{CaF}_{2} \text { signal }}
$$

where the signal due to $\mathrm{CaF}_{2}$ takes into ascount the corrections required for scattering effects. The absorption coefficient calculated from the relative absorption can be fitted to Melamed's function and the RG theory. The good fit to Melamed's function at low $\beta$ values highlights the importance of scattered light in the PAS signal, while at high absorption coefficients (specially near the saturation region) RG theory is valid. These results seem to indicate that in the intermediate regions, the RG theory may not be valid.

If the sample is very thin $\left(d \ll \mu_{s}\right)$ the photoacoustic signal would be independent of the thermal property of the sample since the dissipated heat will be coupled to the gas irrespective of the location in the solid at which the optical energy is absorbed. McClelland and Kniseley (1976a) have therefore suggested preparation of samples by supporting them in finely dispersed from similar to that in electron microscopy. These authors have also discussed the role of the sample and the sample cell on the photoacoustic wave form.

Wetsel and McDonald (1977) using the composite piston model discussed earlier have obtained the absolute absorption coefficient of a dye solution at a particular wavelength. The values obtained by them were in excellent agreement with transmission measurements. Since an accurate determination of the length of the sample is an important parameter in this method, it seems to have limited applicability.

Roark et al (1978) have used the photoacoustic phase angle spectroscopy ( $\phi$ PAS) to obtain quantitative absorption spectra of samples in condensed phase. In RG theory, $\phi$ is given by

$$
\phi=\tan ^{-1}[\operatorname{Im}(Q) / \operatorname{Re}(Q)],
$$

where the quantity $Q$ is the complex amplitude of the sinusoidal variation of the temperature in the gas phase due to the photoacoustic effect and is given by (6) 
and (8). They assume the absorptivity to be constant throughout the sample. The solution for $\phi$ then takes the form,

$$
\phi=\tan ^{-1}\left[\frac{\left(-B^{3}+2 B-4\right)}{\left(B^{3}-2 B^{2}+2 B\right)}\right] \text {, }
$$

where $B$ is defined as $B=\beta / a_{0}=\beta(2 \alpha / \omega)^{1 / 2}$. We seo that $\phi$ is independent of the light intensity, sample reflectivity and luminescence quantum yield. The graphic solution of the above curve shows that significant phase angle changes occur upto $B \approx 10$. Roark et al point out that this is well above the value for which saturation in the PAS amplitude sets in and that to obtain saturation-free spactroscopy using only the amplitude of the photoacoustic signal would require a 100-fold increase in the modulation frequency and thus more than two orders of magnitude decrease in signal strength.

The experimental value of the phase angle, $\phi_{\text {oxp }}$ is not that corresponding to (15), but includes a relative phase angle shift $\phi_{0}$ due to instrumental parameters and phase shifts due to cell resonances, the positioning of the sample with respect to the light beam and other factors. Roark et al (1978) determined $\phi_{0}$ by determining the $\phi_{\text {exp }}$ for a sample whose absorption coefficient is known at a particular wavelength. $\phi_{0}$ was then assumed to be constant for the rest of the region of the spectrum. The absorption spectrum of $\mathrm{Fe}$ (bipy) $)_{3} \mathrm{Br}$ in aqueous solution obtained by the transmission method and the spectrum obtained by $\phi$ PAS at various frequencies are shown in figure 19.

Poulet et al (1980) have used the composite piston model and $\phi$ PAS to render PAS into a quantitative technique for thermally thick solutions. These workers have also examined the variation of the PAS signal amplitude with $\beta \mu$, from a solution of neutral red. The amplitude becomes independent of $\beta$ only when $\beta \mu_{0} \approx 10$ which is in agreement with the RG theory. This seems to have been overlooked by Roark et al (1978) when they asserted the superiority of $\phi$ PAS. The advantage in using $\phi$ PAS therefore seems to be subject to some doubt. For low values of $\beta$, Poulet et al find that the experimental results are more in agreement with the mechanical piston model of McDonald and Wetsel (1977).

We have examined the normalized PAS spectra of $\mathrm{As}_{60} \mathrm{Se}_{40}$ as well as the phase dependence of its PA signal at $11.6 \mathrm{~Hz}$ and found that the change in the phase actually saturates before the intensity and that at longer wavelength (or smaller absorption coefficients) the phase does not show change but there is a large change in intensity (figure 20). These results seem to indicate that there may be no correlation between the phase and the amplitude, especially at low $\beta$ values. When an external sound (due to the chopper) at the same frequency but at a different phase was introduced into the cell, the phase angle changes sharply between 760 and $800 \mathrm{~nm}$. This change is not present in the absence of the external noise (using an unperforated perspex chopper wheel). The absence of correlation between the phase change and the intensity could be due to an impurity in the sample or due to two signal sources as discussed earlier. The sharp change in the phase angle at low values of the absorption coefficient due to the introduction of an external sound is interesting and has not been noticed by earlier workers. It seems to us that the introduction of an external aconstic signal into the cell at the same frequency could be exploited to measure $\phi$ PAS of samples with low absorption coefficients. 


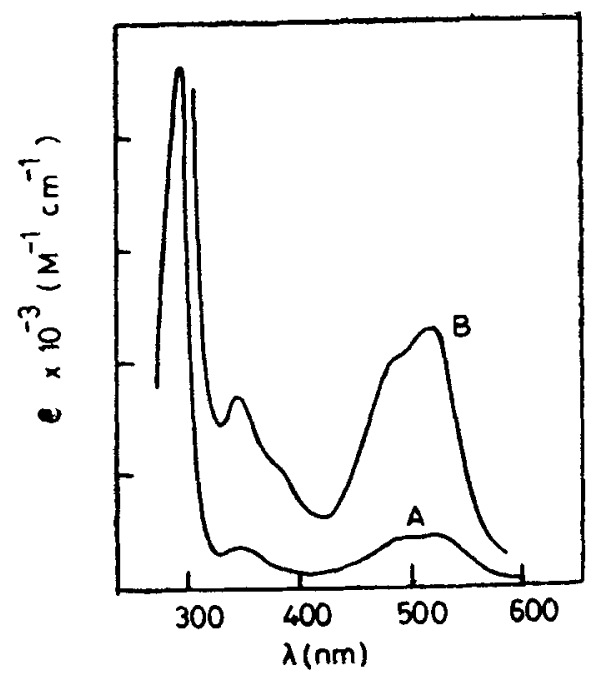

(a)

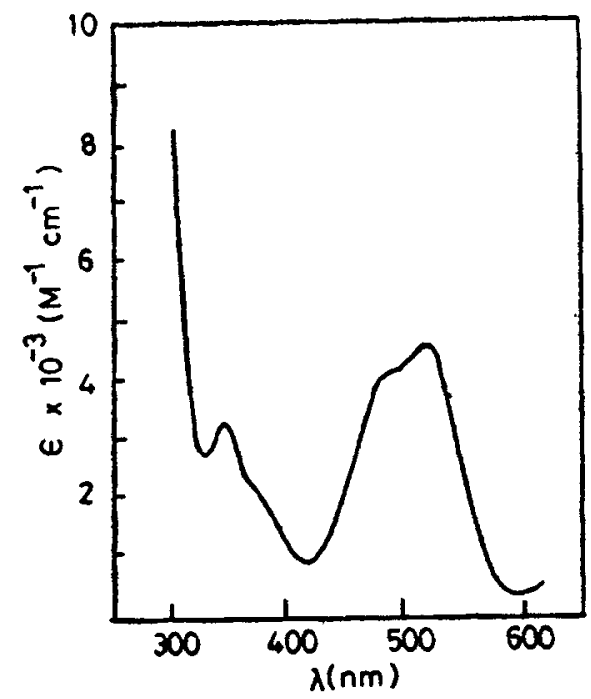

(b)

Figure 19. (a) Transmission optical spectrum of $\mathrm{Fe}(\text { bipy })_{3} \mathrm{Br}_{2}$ solution (curve $\mathrm{A}$ ) ; Curve $B$ is obtained after ten-fold magnification. (b) Absorption spectrum obtained from the phase dependence at $250 \mathrm{~Hz}$.

An observation that is somewhat puzzling to us is that we obtain a $\omega^{-1}$ dependence for this sample at all wavelengths below $800 \mathrm{~nm}$. In terms of RG theory, this would indicate that we are operating in the region $\mu_{\beta}<\mu_{z}<10 \mu_{\beta}$ in the wavelength region $600-800 \mathrm{~nm}$. However, the absence of any phase dependence between 800 and $760 \mathrm{~nm}$ (in the absence of external sound at same frequency) (see figure 20) suggests that $\beta$ values are rather low and in this wavelength region at least $\mu_{\beta}$ is likely to be greater than $\mu_{s}$. The amplitude and phase variation may also arise out of unknown surface effects. 


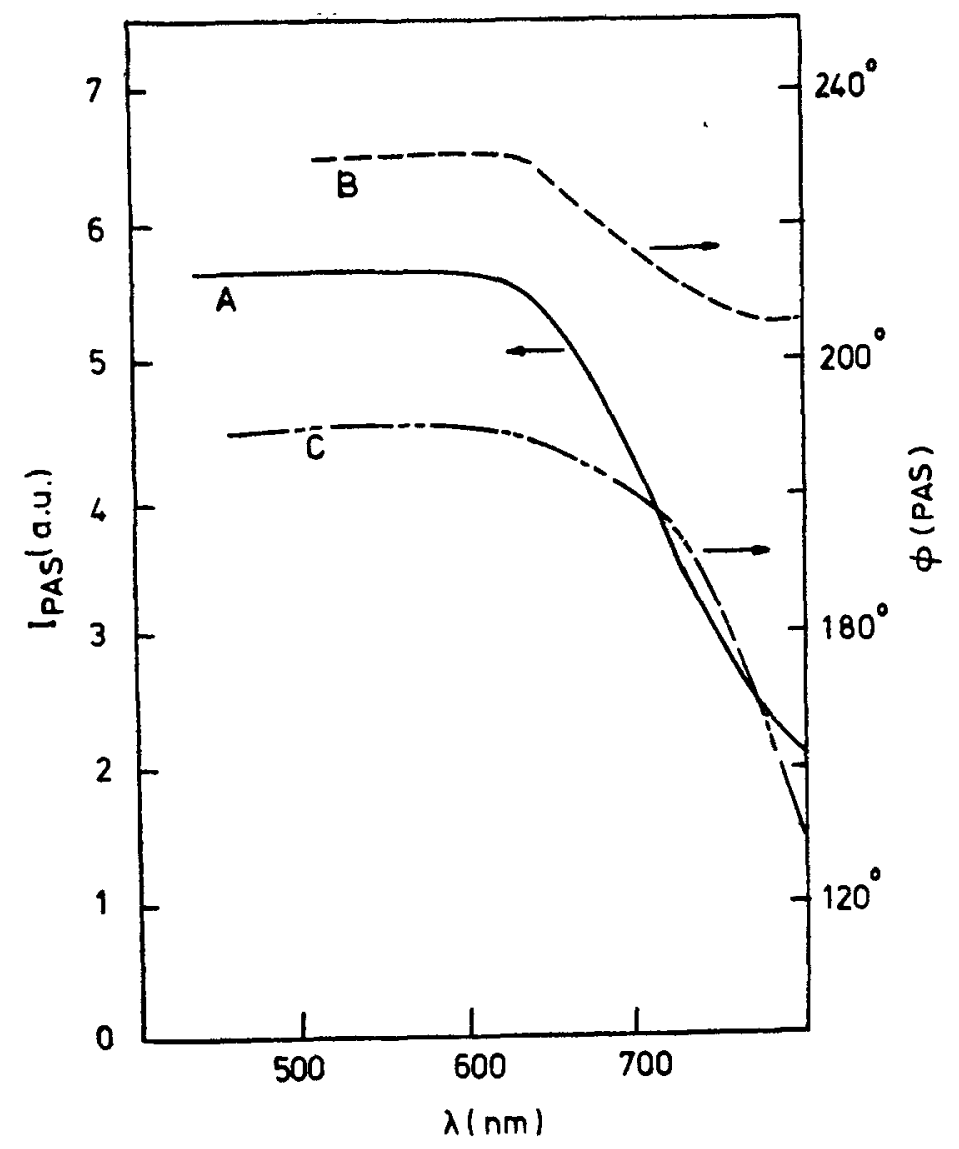

Figure 20. PAS of As se $_{\infty 0}$ glass : A, normalised PAS signal ; B, $\phi$ (PAS) us $\lambda$ plot; $\omega=11.6 \mathrm{~Hz} ; \mathrm{C}, \phi(\mathrm{PAS})$ vs $\lambda$ plot, $\omega=71.3 \mathrm{~Hz}$, chopper noise present in tho cell.

\section{Studies of solids}

The photoacoustic spectrum of $\mathrm{Ho}_{2} \mathrm{O}_{3}$ was shown earlier (figure 13) to illustrate the performance of the spectrometer fabricated by us. A comparison of the spectrum of $\mathrm{Ho}_{2} \mathrm{O}_{3}$ with that of $\mathrm{HoAlO}_{3}$ recorded under identical conditions showed differences in intensities of some of the lines and absence of certain other lines. Similar differences are seen in the spectra of $\mathrm{Nd}_{2} \mathrm{O}_{3}$ and $\mathrm{NdAlO}_{3}$ (figure 21). It is known that $\mathrm{Ho}_{2} \mathrm{O}_{3}$ containing a small amount of $\mathrm{Co}^{2+}$ exhibits additional lines due to fluorescence quenching (Rosencwaig 1976). PAS can be effectively employed to examine such differences in the electronic transitions of systems where some of the transitions are associated with a greater probability for radiative decay. We shall discuss the study of fluorescence yields and life times by PAS in the next section.

In figure 22 we show the normalized spectrum of a commercial sample of $99.9 \%$ $\mathrm{Cr}_{2} \mathrm{O}_{3}$ as well as the phase dependence of the signal. Although the peak positions correspond to those mentioned in the literature, the intensities are different 


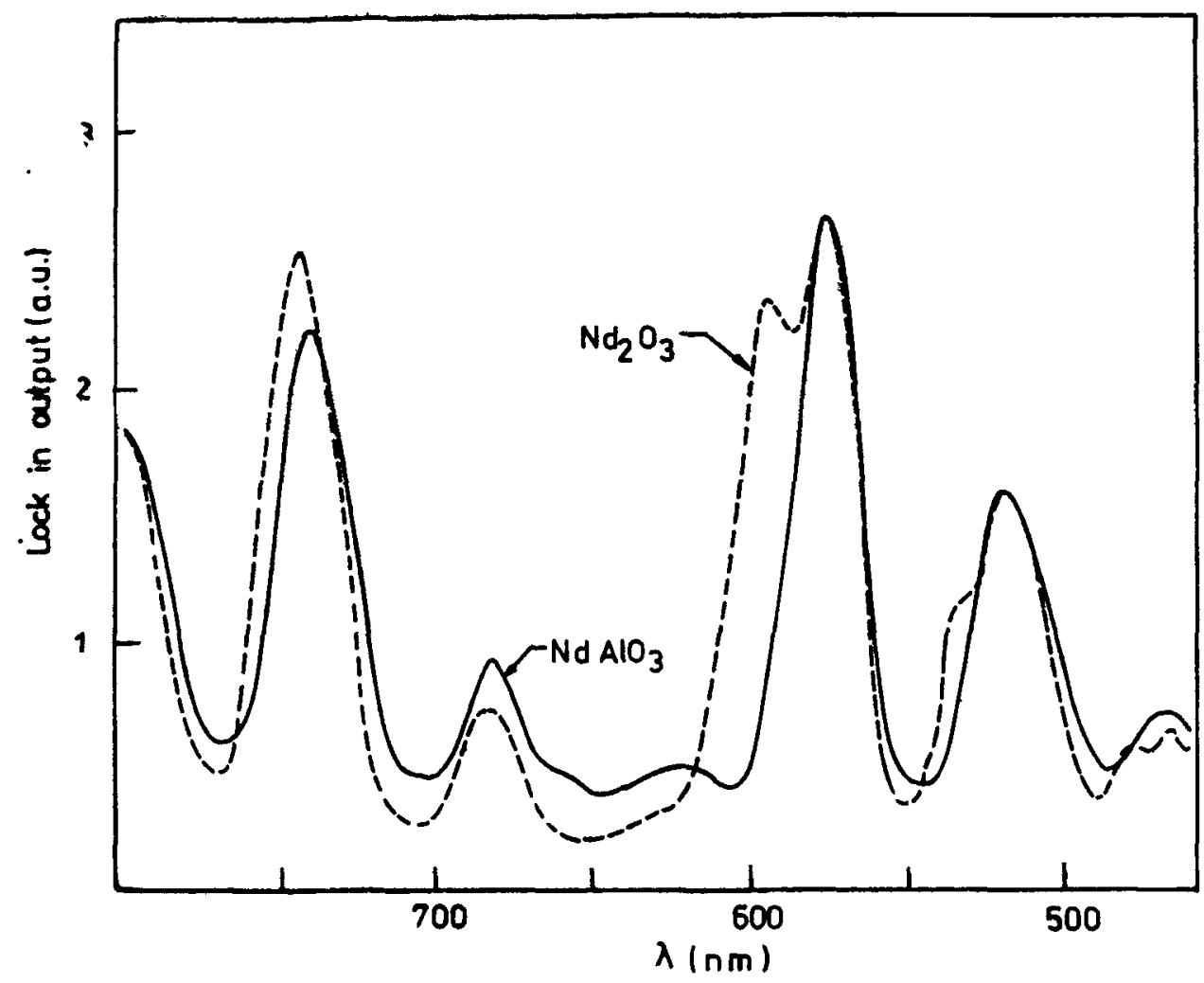

Figure 21. Uncorrected PA signal from $\mathrm{Nd}_{2} \mathrm{O}_{3}$ and $\mathrm{NdAlO}_{8}: \omega=11 \cdot 6 \mathrm{~Hz}, \Delta \lambda-$ $10 \mathrm{~nm}$, Drive $=100 \mathrm{~nm} / \mathrm{min}$. For power spectrum see Fig. 16 (curve C).

from those reported by Rosencwaig (1976) or Adams et al (1976). This may be due to signal saturation effects which would give rise to different intensities at different frequencies. We have, however, not been able to change the nature of the spectrum by increasing the chopping frequency. We also note that the phase is much less sensitive to the features of the absorption spectrum. Murphy and Aamodt (1977b) have found that the ratio of the ${ }^{4} A_{2} \rightarrow{ }^{4} \mathrm{~T}_{2}\left(\triangle E \approx 18000 \mathrm{~cm}^{-1}\right)$ intensity to the ${ }^{4} A_{2} \rightarrow{ }^{4} A_{1}\left(\triangle E \approx 25000 \mathrm{~cm}^{-1}\right)$ intensity in $\mathrm{Cr}_{2} \mathrm{O}_{3}$ to be $6: 4$ which is in agreement with transmission data from single crystals. The ratio found by us is much less. In the spectrum of reduced $\mathrm{Cr}_{2} \mathrm{O}_{3}$ supported on alumina where a monolayer is expected to be present, the ratio is still less. A possible explanation is that there are appreciable amounts of $\mathrm{Cr}^{6+}$ on the surfaces. In the spectrum of the sample prepared by sintering chromia gel at $800^{\circ} \mathrm{C}$ (Curve $\mathrm{C}$ ), the base line has shifted considerably as the sample was visibly more black in colour (due to presence of different oxidation states including +6 ). Curve $D$ in the same figure shows the spectrum of chromia gel. The spectrum does not show good resolution of bands. What is of interest, however, is that the spectrum of the gel could be obtained.

In figure 23 the PAS spectrum of $\mathrm{LaCrO}_{3}$ is compared with the spectra of $\mathrm{YCrO}_{\text {, }}$ (and $\mathrm{La}_{2} \mathrm{AlCrO}_{6}$ ). We see that $\mathrm{YCrO}_{3}$ shows an additional band prominently. 


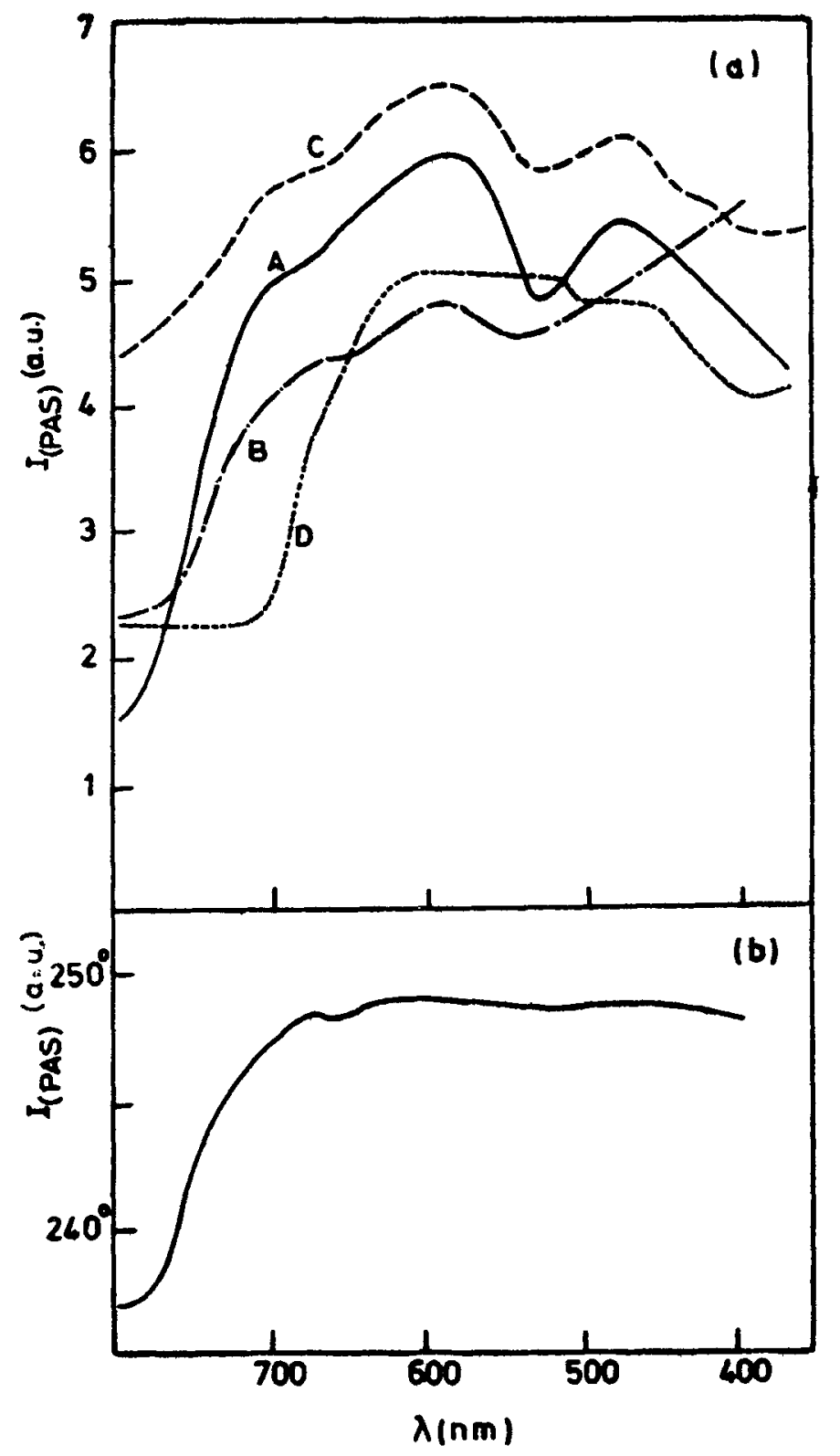

Figure 22. (a) Normalised $\mathrm{PA}$ spectra of $\mathrm{Cr}_{2} \mathrm{O}_{3}: \quad \mathrm{A}, 99.9 \%, \mathrm{Cr}_{2} \mathrm{O}_{3} ; \mathrm{B}, 2 \%$ $\mathrm{Cr}_{2} \mathrm{O}_{3}-\mathrm{Al}_{2} \mathrm{O}_{3}$ (reduced) ; $\mathrm{C}, \mathrm{Cr}_{2} \mathrm{O}_{3}$ obtained by the decomposition of chromia gel at $800^{\circ} \mathrm{C}$; D, chromia gel. (b) Phase angle change for $\mathrm{Cr}_{2} \mathrm{O}_{3}$ (99.9\%) powder $\omega=11 \cdot 6 \mathrm{~Hz}, \Delta \lambda=10 \mathrm{~nm}$.

We also see that the crystal-field splitting $\left({ }^{4} A_{2} \rightarrow{ }^{4} T_{2}\right)$ energy of $\mathrm{Cr}^{3+}$ is higher in $\mathrm{YCrO}_{3}$ as expected. The spectrum of $\mathrm{La}_{2} \mathrm{AlClO}_{6}$ is considerably different and the CT band in this oxide is at lower energies. We have also studied the spectra of $\mathrm{Fe}_{2} \mathrm{O}_{3}, \mathrm{LaFeO}_{3}, \mathrm{La}_{2} \mathrm{AlFeO}_{6}$ and $\mathrm{K}_{6} \mathrm{Fe}(\mathrm{CN})_{6}$ (figure 24). Spectra of $\mathrm{Fe}_{2} \mathrm{O}_{3}$

P.(A) -3 


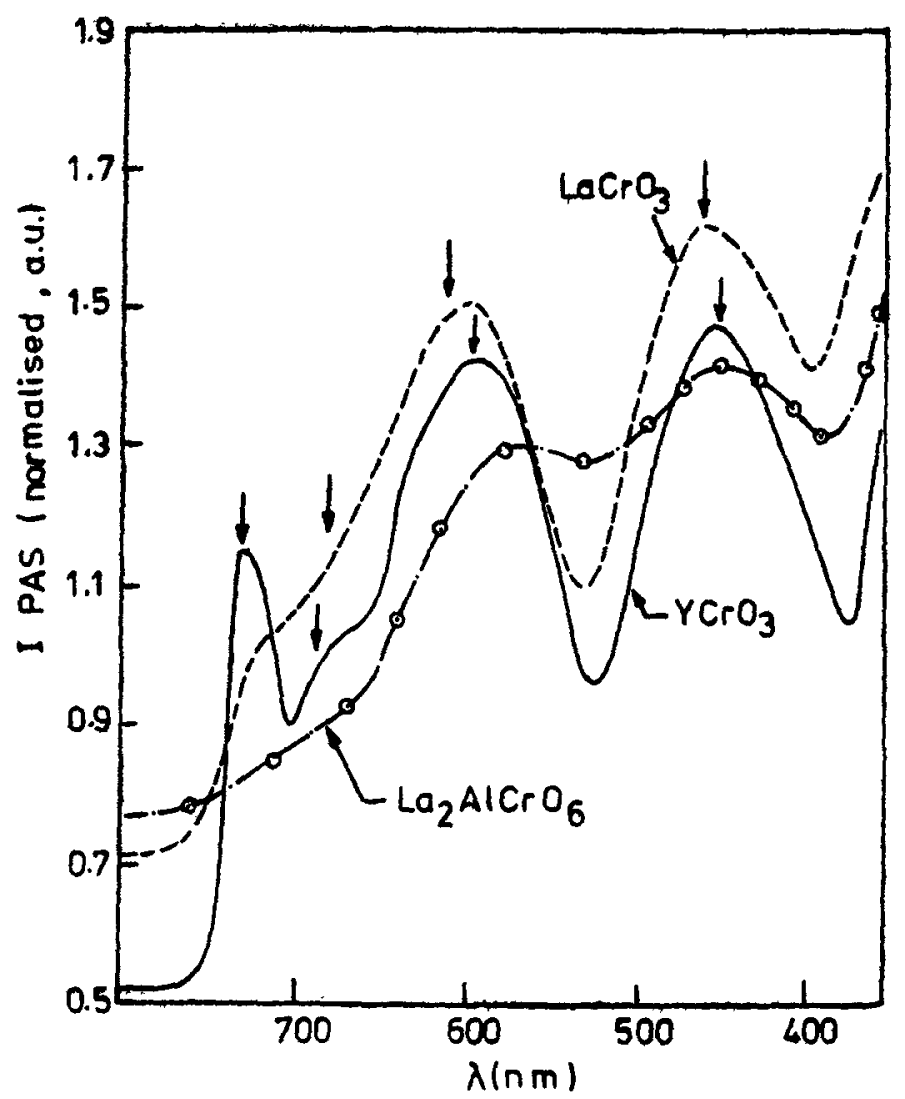

Figure 23. Normalized $\mathrm{PA}$ spectra of $\mathrm{LaCrO}_{3}, \mathrm{YCrO}_{8}$ and $\mathrm{La}_{4} \mathrm{AlCrO}$. Arrows represent positions of peaks from the literature.

and $\mathrm{LaFeO}_{3}$ show peaks due to the expected transitions. The ${ }^{\circ} A_{10} \rightarrow{ }^{4} T_{2 g}$ transition (around $700 \mathrm{~nm}$ in $\mathrm{LaFeO}_{3}$ ) is however absent in $\mathrm{La}_{2} \mathrm{AlFeO}_{6}$. Instead, there seems to be a strong CT band which is shifted to lower energies just as in $\mathrm{La}_{2} \mathrm{AlCrO}_{6}$. We have obtained spectra of compounds like $\mathrm{LaAl}_{0 \cdot 85} \mathrm{Fe}_{0.5} \mathrm{O}_{8}$ where $\mathrm{Fe}^{8+}$ is present in small concentrations. We have also been able to record the spectrum of $\mathrm{PbCrO}$, which is an intensely coloured dye. Spectra of such intensely coloured substances are difficult to obtain by optical spectroscopic methods.

We have investigated the spectra of a series of glasses of the composition $\mathrm{As}_{1-}$, $\mathrm{Se}_{\mathrm{w}}$ which are highly reflecting and absorbing. Reliable spectra of such materials are difficult to obtain by transmission or reflectance spectroscopy. Absorption edges of such materials should be associated with intensity maxima, but the maxima, if at all found, are broad (figure 25). Flat maxima in the absorption edge measurements of several materials have been reported by Wong (1980). We are not certain to what extent saturation effects are responsible for this observation.

The spectra of amorphous and crystalline $\mathrm{As}_{2} \mathrm{~S}_{3}$ are compared in figure 26 . We see the tailing of the absorption edge prominently in amorphous $\mathrm{As}_{2} \mathrm{~S}_{3}$ while 


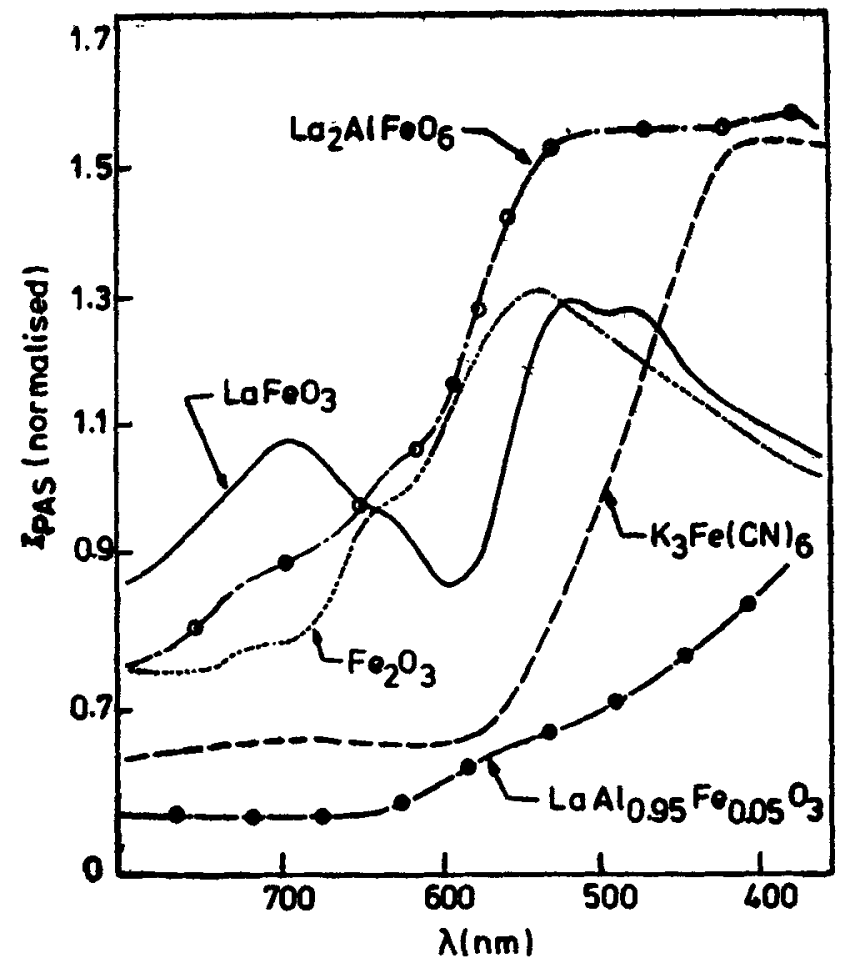

Figure 24. Normalized PA spectra of $\mathrm{LaFeO}_{3}, \mathrm{Fe}_{2} \mathrm{O}_{3}, \mathrm{La}_{2} \mathrm{AlFeO}_{4} \mathrm{LaAl}_{0.055} \mathrm{Fe}_{0.05} \mathrm{O}_{8}$ and $\mathrm{K}_{\mathrm{g}} \mathrm{Fe}(\mathrm{CN})_{6}$.

crystalline $\mathrm{As}_{2} \mathrm{~S}_{3}$ has a sharp absorption edge. The maximum in the absorption edge is quite prominent in both these cases. The band edges of $\mathrm{As}_{2} \mathrm{~S}_{3}$ and $\mathrm{As}_{2} \mathrm{Se}_{3}$ shift to lower energies in the amorphous state.

We have examined the spectra of $\mathrm{KNiF}_{3}$ and $\mathrm{KGu}_{0.3} \mathrm{Ni}_{0.7} \mathrm{~F}_{3}$ prepared by the reaction of concentrated metal chloride solutions with a $K F$ solution acidified with HF. The compounds were single phase, but we could not obtain their transmission optical spectra because of the scattering due to the highly divided nature of the samples. PAS of the two compounds were nearly identical with respect to peak positions, but the copper substituted compound showed a lower absorption between 500 and $600 \mathrm{~nm}$.

We have tried to obtain the spectra of dark compounds such as $\mathrm{Ti}_{2} \mathrm{O}_{3}$. In such cases we find saturation at all the wavelengths studied $(400-800 \mathrm{~nm})$ and reduction in particle size failed to improve the situation. To our knowledge, no case is reported where saturation effects have been removed by increasing the chopping frequency (although this is expected from the RG theory).

\subsection{Phase transitions}

Pichon et al (1979) have studied phase transitions in solids at low temperatures employing PAS. For thermally thick samples, the PA signal is proportional to $\left(\mu_{o} / T_{o}\right)\left(2 / \rho_{s} C_{s}\right)$ where $C_{s}$ is the specific heat of the solid. Under this condition, 


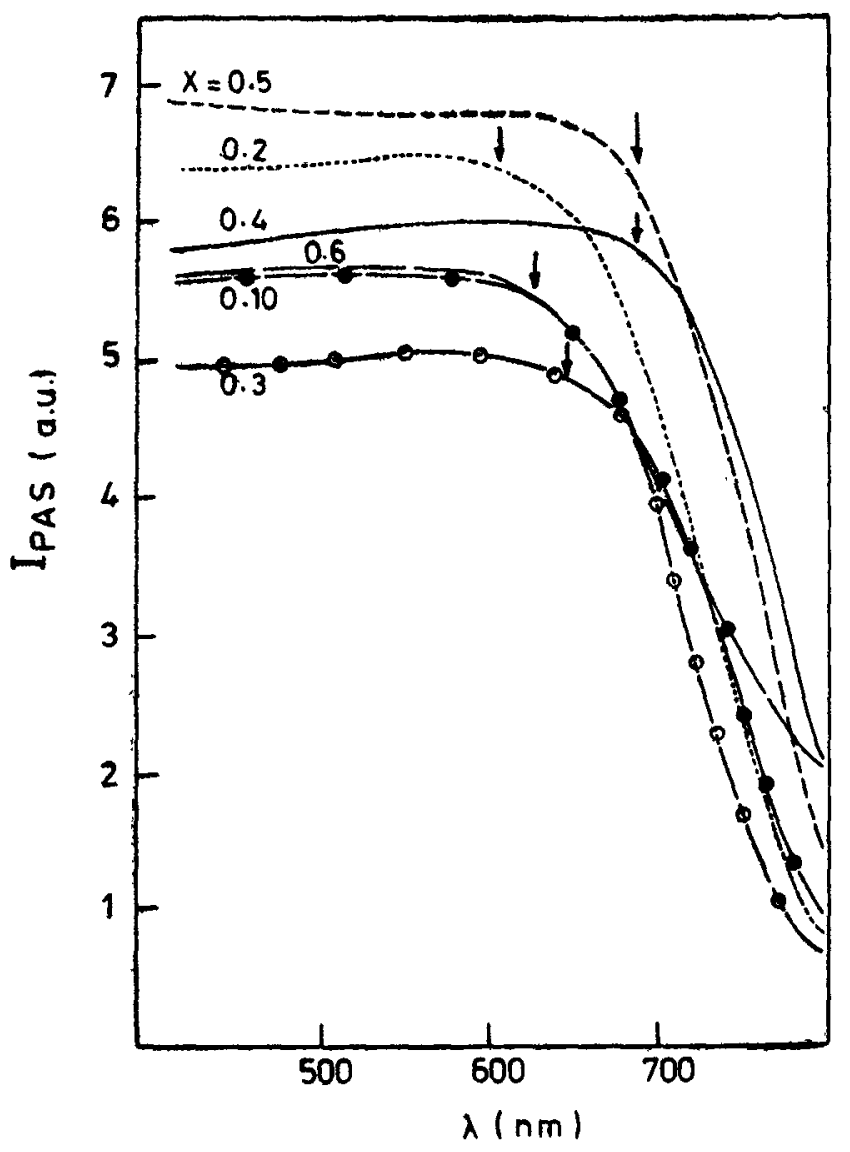

Figare 25. Normalized PAS of $\mathrm{As}_{1,}, \mathrm{Se}$, glasses. Arrow indicates the wavelength at which there is no further chango in the phase of the signal.

the sample contribution to the signal varies as $1 / C_{z}$. Pichon et al studied magnetic phase transitions in $\mathrm{CrCl}_{3}$ and $\mathrm{MnF}_{2}$ by recording the PA signal as a function of temperature and obtained specific heat maxima at the calorimetric temperatures. Florian et al (1978) studied the PA signal of gallium through its melting point and found the amplitude and phase to be different in the liquid and solid phase. These workers have been able to localize the phase transition temperatures in transparent liquids and solids by the introduction of the so-called photoacoustic probes. The freezing point of water and the phase transition in $\mathrm{K}_{2} \mathrm{SnCl}_{6}$ were thus detected by them. Korpium et al (1980) have examined the temperature dependence of the photoacoustic signal at the solid-liquid transition of gallium at $302.8 \mathrm{~K}$ and the phase transition of $\mathrm{VO}_{2}$ at $340 \mathrm{~K}$. Siquiera et al $(1980)$ have also demonstrated experimentally the usefulness of the photoacoustic effect for investigating phase transition in solids. They have completely characterized the cell by using germanium which has well-known thermal properties. 

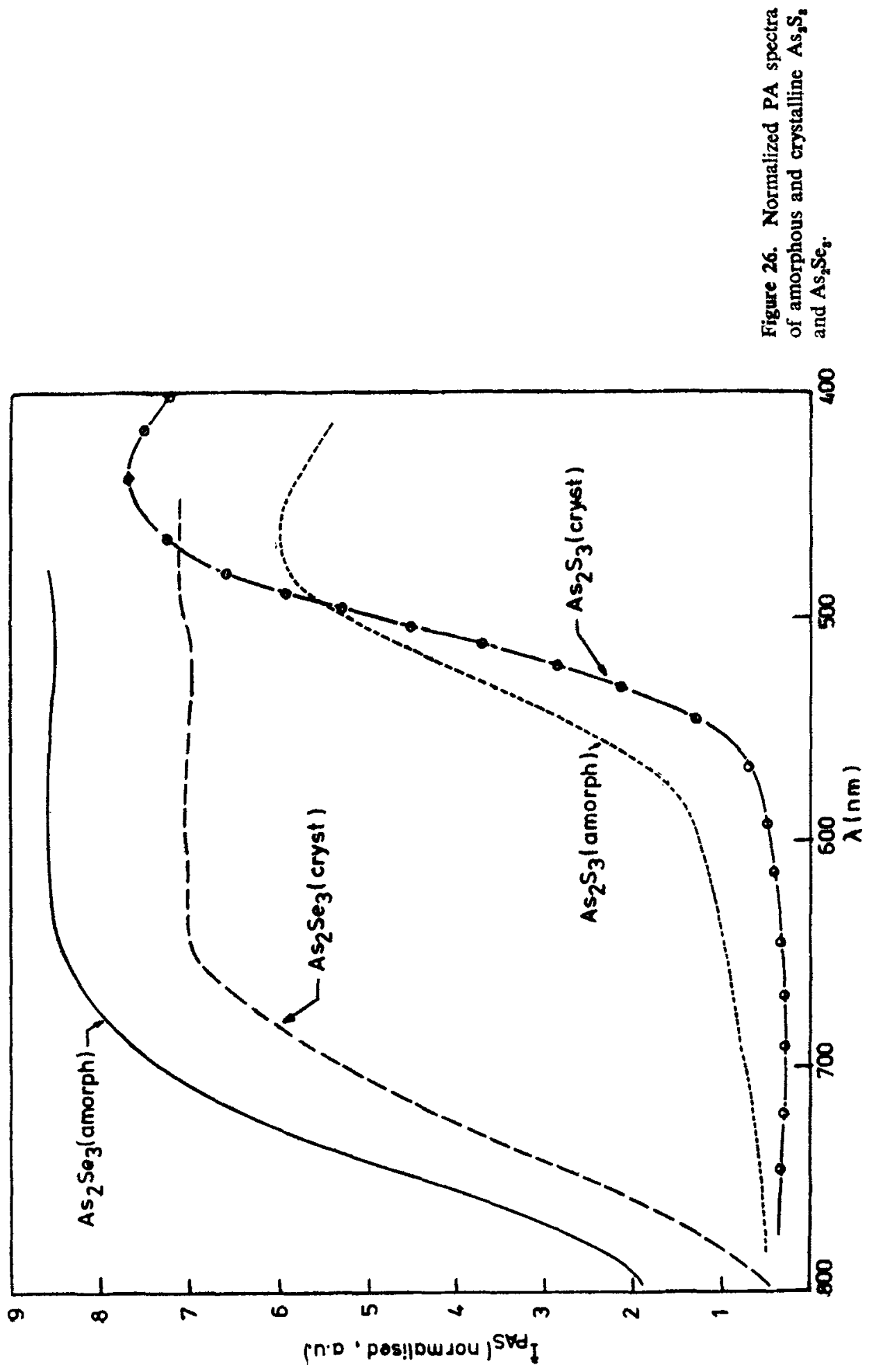
In figure 27 our results on the temperature dependence of the PA signal of VO are shown. White light of very low intensity using a water filter was used for the studies. The phase transition temperature is reflected in the PA signal intensity variation. The transition temparature was $340 \mathrm{~K}$ in the heating cycle and $336 \mathrm{~K}$ in the cooling cycle reflecting the behaviour found by DTA or DSC. Although $\mathrm{VO}_{2}$ is known to show changes in its optical absorption spectrum, we find no significant change in the baseline. In figure 28 we show the temperature dependence of the PA signal of NiO. A rather sharp reversible minimum in the PA signal is found at $520 \pm 2 \mathrm{~K}$ corresponding to the Neel temperature. The ease with which we have been able to detect the Neel temperature is amazing. In this case, it is unlikely that the intensity of optical absorption reaches a maxi. mum at the Neel temperature. The change in the PA signal is likely to be due to changes in the specific heat. Since the PA signal in these cases is produced by the absorption of energy by the electronic levels, it seems possible that PAS would be a good technique to study electronic specific changes. We also show in figure 28 the amplitude of the PA signal of $\left[\left(\mathrm{C}_{2} \mathrm{H}_{5}\right) \mathrm{NH}_{3}\right]_{2} \mathrm{CuCl}_{4}$ which shows a structural phase transition around $360 \mathrm{~K}$. We see a discontinuity in the signal rather than a peak. We have employed the PAS technique to detect the weak phase transition in $\mathrm{La}_{2} \mathrm{CuO}_{4}$ at $533 \mathrm{~K}$ which is not seen by DTA and DSC. We have also been able to detect the glass transition in $\mathrm{As}_{40} \mathrm{Se}_{60}$ by PAS.

McGlelland and Kniseley (1979) have demonstrated the potential of the photoacoustic technique for observing changes in the crystallinity of semiconductors due to annealing. A decrease is observed when the PA signal is monitored during a scan across a disordered region to a single crystal region with an abrupt change in the demarcation region. The results are reasonably consistent with the onedimensional RG model. These authors do not observe a one-to-one quantitative correspondence with the loss of the reflectivity signal to the gain in the PA signal in the two phases.

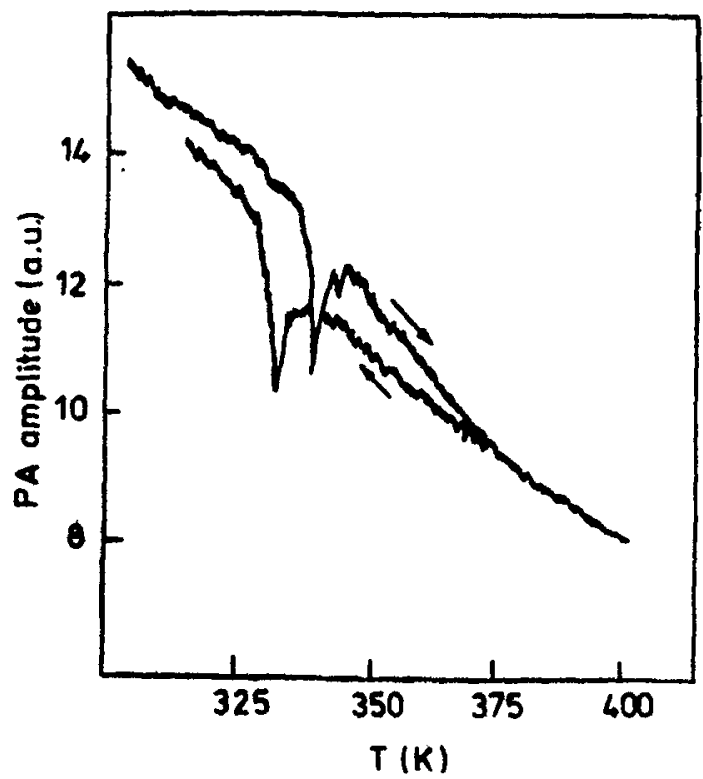

Figure 27. Photoacoustic signal from $\mathrm{VO}_{2}$ as a function of temperature. White light from a tungsten-halogen lamp was used. 


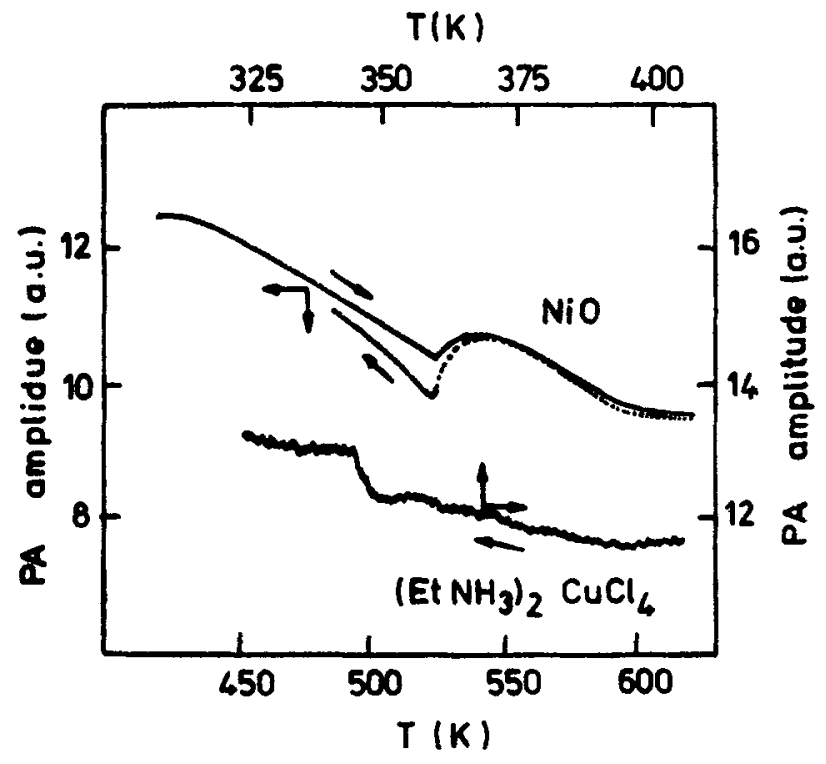

Figure 28. PA signal from $\mathrm{NiO}$ and $\left(\mathrm{EtNH}_{2}\right)_{2} \mathrm{CuCl}_{4}$ as a function of temperature.

\section{Fluorescence efficiencies and lifetimes}

Since the photoacoustic signal is sensitive only to the heat deposited in the sample, any absorbed energy given out as fluorescence does not coniribute to the signal. Photoacoustic spectroscopy should therefore provide information concerning the balance between radiative and non-radiative processes in solids. This enables the study of quantum efficiency and lifetimes of fluorescent levels. Rockley and Waugh (1978) measured the fluorescence yields of dye solutions, $\phi_{f}$, by the analysis of fluorescence spectra. The PA signal being proportional to the total heat emitted by the sample, the intensity of the PAS signal from the higher excited $S_{n}$ and lower excited $S_{1}$ states may be written as

$$
I_{\text {PAS }}\left(S_{n}\right)=k\left[E\left(S_{n}\right)+E\left(S_{1}\right)\left(1-\phi_{t}\right)\right]
$$

and

$$
I_{\mathrm{PAS}}\left(S_{1}\right)=k\left[E\left(S_{1}\right)\left(1-\phi_{f}\right)\right]
$$

where $k$ is the proportionality constant. Since, $E\left(S_{n}\right), E\left(S_{1}\right)$ are known quantities while $I_{\mathrm{PAS}}\left(S_{n}\right)$ and $I_{\mathrm{PAS}}\left(S_{1}\right)$ are measured quantities, the two unknown $k$ and $\phi_{\text {, }}$ may be obtained from the above equations.

Powell et al (1980) have used the above technique for determining quantum efficiencies of $\mathrm{Nd}^{3+}$. These workers have also used the phase dependence to obtain a measure of the lifetimes of the excited states (also see Murphy and Aamodt 1977b). The fraction $a(\lambda)$ of absorbed light converted to heat at wavelength $\lambda$ is given by three ratios : branching ratio $b_{i j}=-\mathbf{k}_{i j} / \mathbf{k}_{j j}$, where $\mathbf{k}_{b j}$ is the interlevel transition rate, $\epsilon_{i}^{(b)}=E_{i} / E_{k}$, and non-radiative/total-transition rate ratio $\mu_{i j}=\gamma_{t j} /$ $\mathbf{k}_{i j}$. In terms of these quantities,

$$
a_{i}=\sum_{j, m} \epsilon_{j}^{(0)} \mu_{j m} a_{m^{3}} \text {, }
$$


the summation over $m$ being carried out for all levels where $k_{m m} \neq 0$. In the above equation, $a_{i j}=b_{i j}(i \neq j)$ and $a_{i j}=-\left(1+i \omega / k_{i i}\right)$. The frequency dependence of $a$ enters only through the terms $a_{i i}$. Hence, for each energy level, the frequency dependence separates into a low- and high-frequency regime with crossover at $\omega=k_{i i}$. The various rates $k_{i j}$ can then be studied individually by choosing an appropriate modulation frequency. This is especially suitable when the rates are sufficiently separated and if the sample frequency dependence can be isolated from the cell frequency dependence.

The expression for the PAS signal after excitation in energy level $E_{i}$ at the phase angle $\theta$ is

$$
I_{a}(\theta)=C\left(P_{a} / E_{a}\right) \quad \sum_{i j} \phi_{i j}^{n f} E_{i j} \cos \left[\left(\psi+\tan ^{-1} 2 \pi \omega \tau_{i}\right)-0\right],
$$

where $\tau_{i}$ is the lifetime of the level, $P_{a}$ the power absorbed, $C$ a factor accounting

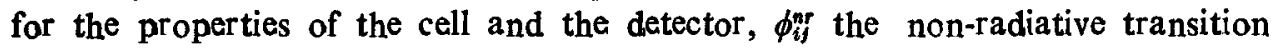
probabilities between levels $i$ and $j$ with energy $E_{i}, \tan ^{-1}\left(2 \pi \omega \tau_{i}\right)$ the phase shift of the signal due to the lifetime of the initial level and $\psi$ the phase shift due to the detection procedure. Powell et al (1978) determined the quantum efficiency by taking the ratio of two photoacoustic signals as suggested by Rockley and Waugh (1978). The maximum PA intensity and the phase angle at the signal maximum were measured for two different wavelengths of excitation (4765 and $5145 \AA$ argon laser line at two different chopping frequencies $(\omega=312$ and $1000 \mathrm{~Hz})$. The quantum efficiency $Q E$, probability of non-radiative relaxation $P_{N B}$ and the probability $\boldsymbol{P}_{\mathbf{X}}$ of concentration quenching of the metastable states are given by,

$$
\begin{aligned}
\mathrm{QE} & =W_{R} /\left(W_{R}+W_{N R}+W_{x}\right), \\
P_{N R} & =W_{N R} /\left(W_{R}+W_{N R}+W_{X}\right),
\end{aligned}
$$

and $\quad P_{X}=W_{X} /\left(W_{R}+W_{N R}+W_{X}\right)$,

so that $\mathrm{QE}+P_{N R}+P_{X}=1$. It is assumed that only the metastable level has a long enough lifetime to introduce a measurable phase shift into the signal. The unknown experimental factors are eliminated by taking the ratio of the PA signals at two different excitation frequencies and $\psi$ is solved for in an iterative manner using the two chopping frequencies. The value of the quantum efficiency in the absence of concentration quenching can be found from the expression QE (0) $=\mathrm{QE}(x) \tau_{0} / \tau_{\text {。 }}$ where $x$ is the concentration. Quimby and Yen (1980a) have measured the quantum yield of the ruby fluorescence by a technique involving the measurement of the phase of the photoacoustic signal as a function of the chopping frequency which is applicable to simple decay schemes with sufficiently long fluorescence lifetimes.

Merkle and Powell $(1977,1978)$ have used the PAS phase to measure the lifetime associated with non-radiative de-excitation paths employing the relationship between the non-radiative lifetime and the phase shift for a gaseous sample derived by Harshbarger and Robin (1973). Thus, the radiationless relaxation rates of $\mathrm{Eu}^{2+}$ in a $\mathrm{KCl}$ matrix has been examined. A large relative phase shift was observed between the PAS signal resulting from excitation in the $t_{2}$ and $e_{g}$ bands of the $\mathrm{Eu}^{2+}$ ion and was attributed to a long-lived excited state of $4 f^{\circ}$ configuration. Merkle and Powell observe differences in the maxima of the photoacoustic spectra 
at different phase angles for the two strong transitions of $\mathrm{Eu}^{2+}$ in $\mathrm{KCl}$ matrix from the lower ${ }^{8} S_{7 / 2}\left(4 f^{7}\right)$ ground state to $e_{g}$ and $t_{20}$ compcnents of the $4 f^{8} 5 d$ configuration. They assume that the phase angle at which the signal is maximum is related to the lifetime of the state. The peak intensity of the PAS signal in the high energy band occurs at a phase shift of $35^{\circ}$ (for $\omega=100 \mathrm{~Hz}$ ) relative to the low lying energy band which occurs at $0^{\circ}$ phase shift. They accounted for this discrepancy by assuming that a fraction of the $e_{0}$ electrons relax to a ${ }^{8} P_{7 / 2}\left(4 f^{7}\right)$ state which has a lifetime of $3.6 \mathrm{~m}$, a fraction of which relaxes non-radiativly io contribute to the phase shift.

Peterson and Powell (1978) have employed a similar approach to compare the decay modes for $\mathrm{Cr}^{3+}$ ions in $\mathrm{Al}_{2} \mathrm{O}_{3}, \mathrm{MgO}, \mathrm{SrTiO}_{3}$ and $\mathrm{BaTiO}_{3}$. They have shown that it is possible to identify the dominant roles of radiationless relaxation of ions in crystals by comparing photoacoustic spectra with fluorescence excitation spectra. Murphy and Aamodt (1977) have applied the rate-equation model mentioned above for interpreting the observed concentration dependent metastable level quenching rates in ruby.

Quimby and Yen (1980b) have examined RG theory taking into account the spatial migration of energy. In most studies on relaxation rates, it is usually assumed that the spatial migration of the excitation energy is negligible. Systems with a high concentration of optically active centres may exhibit spatial energy migration over several microns.

Starobogatov (1977) has determined the luminescence efficiency using the PAS technique by assuming that the energy of the excited state is lost only by radiative or non-radiative transition. It was shown by Starobogatov that the amount of absorbed energy liberated as heat is given by

$$
E_{\text {thormal }}=\eta_{0} \sigma_{t} I_{\mathrm{e}} \tau h\left(v_{\bullet}-v_{0}\right),
$$

where $\eta_{0}$ is the concentration of the solution, $\sigma_{t}$ the cross-section for transition, $I$. the intensity of the exciting radiation, $\tau$ the duration of the illuminating pulse, $v_{0}$ the mean frequency of the luminescer ze spectrum, and $v_{0}$ the frequency of the exciting radiation. It becomes possible to measure the luminescence quantum yield if one knows the amount of exciting energy absorbed and the amount of energy released as heat as a result of non-radiative transitions. Starobogatov (1979) has also shown that it is possible to measure the quantum yield of photochemical reactions by the PAS method by exciting a solution in the first and second absorption bands and to measure the quantum yield for luminescence for the second excited state by the luminescence technique. Razumova and Starobogatov (1977) have applied the PAS method to study the light quenching of luminescence.

In most optically heated samples, the absorbed energy is rapidly converted to heat so that the heat generation per unit volume has the same time dependence as the excitation source. In materials exhibiting phototrapping, however, emitted radiation and energy stored in metastable levels modify the conversion of optical energy to heat. In general, phototrapping delays heat generation. In continuous wave PAS, these delays are seen as phase shifts. In pulsed PAS where detection is in the time domain, these effects are seen as actual delays in thermal conversion. 
Measurement of fluorescent yields and lifetimes have generally been based on the RG theory. To a certain extent, the results have been rewarding. However, one has to exercise caution especially when comparing intensities of two absorption bands with different absorption coefficients. Moreover, other sources of the photoacoustic signal besides that from the thermal acoustic piston can show significant phase differences. Our observation of a significant phase shift even from a monolayer suggests the need for such caution.

We have examined the change in PAS amplitude of fluorescein solution on addition of excess resorcinol which quenches the fluorescence. The ratio of the unquenched to the quenched amplitudes directly gives the fraction of the excited states decaying non-radiatively.

\section{Dichroism}

If the incident light is not intensity modulated but exhibits a periodic change in its polarization, the photoacoustic signal would not be proportional to $\beta$ but to $\Delta \beta=\beta_{1}-\beta_{2}$ where $\beta_{1}$ and $\beta_{2}$ are the absor ption coefficients for the two orthogonal polarizations 1 and 2 . A polarization modulated beam may beconsidered as a superposition of two intensity modulated beams whose phases are shifted by $\pi$ and which correspond to the orthogonal polarizations 1 and 2. On this basis, Fournier et al (1978) have employed a photoacoustic apparatus for dichroism measurements. The linearly polarized light of a $\mathrm{cw}$ dye laser is sequentially set right or left by a Pochets modulator. These circular polarizations may be transformed into a set of linearly orthogonal polarizations by an achromatic quarter-wave plate (Q). An intensity modulated beam may then be obtained using a polarizer (P). Fournier et al (1978) have shown that by switching $P$ and/or $Q$ from the sample beam to the reference beam, it is possible to measure normalized absorption, linear dichroism, circular dichroism. The strong dichroic signal obtained from polarization modulation $\left(\beta_{\pi}-\beta_{\sigma}\right)$ is very close to the signal deduced from the two intensity modulated $\pi$ and $\sigma$ signals for $\mathrm{NdMoO}_{4}$. The axial absorption and magnetic circular dichroism ( $\left.\mathrm{MCD}=\beta_{\sigma+}-\beta_{\sigma_{-}}\right)$photoacoustic signals of $\mathrm{NdMoO}_{4}$ also compare favourably with the measurements from conventional tochniques. In the case of strong dichroism, the usual MCD apparatus does not give a signal proportional to $\triangle A$ because $10^{-} \Delta A$ can no longer be developed to the first order. This, however, is not the case in PA dichroism experiments because the signal is directly related to $\Delta \beta$.

\section{Piezoelectric PAS}

It has long been known that easily detectable elastic waves accompany the absorption of radiation from high-powered light sources such as an electric arc (Michaels 1961) and a pulsed ruby laser (White 1962, 1963). The generation of elastic waves is considered to be due to transient surface heating (White 1963b). Expansion of the surface caused by heating results in a strain. If the heated surface is stress free, the wave amplitude may be small, but if the surface is constrained as by contact with another body, the stresses and the wave amplitude may be high. 
The measurement of the optical spectrum of a solid by the measurement of the amplitude of elastic waves generated in it was carried out by Hordvik and Schlossberg (1977) who placed a strain-transducer such as a piezoelectric device directly in contact with the sample. The absorption coefficient at a fixed wavelength of a laser beam (by a compound of sufficiently high $\beta$ ) was first determined. The photoacoustic cell was then calibrated for the absorption between the laser beam and the piezoelectric transducer. The average absorption coefficients at new wavelengths were then obtained by using laser beams of different wavelength at exactly the same calibration positions.

Hordvik and Schlossberg (1977) used a chopped cw laser and the output of the piezoelectric transducer was linearly related to the power of the laser beam. In their theoretical analysis, these authors used a cylindrical coordinate system to determine the elastic strain (as a function of time and position) generated by a laser beam of Gaussian cross-section propagating through an absorbing solid. When $\beta l \ll 1$, they obtained the relation

$$
\epsilon_{r r}=-\epsilon_{\theta \theta}=-\beta P \alpha_{0}(1+\sigma t) / 2 \pi C \rho(1-\sigma) r^{2},
$$

where $\epsilon_{r r}$ is the radial strain, $\epsilon_{\theta \theta}$ is the azimuthal strain, $P$ the total beam power, $a_{0}$ the thermal expansion coefficient, $\sigma$ the Poisson's ratio, $C$ the specific heat, $\rho$ the density, $t$ the time and $r$ the distance. They predicted and obtained a linear dependence of the signal with chopping frequency over $150-3000 \mathrm{~Hz}$ range and at intermediate distances between the laser beam and the transducer, found an approximate $r^{2}$ dependence in agreement with (21). At shorter distances, the strain is finite at $r=0$ which invalidates the approximation used for the derivation of the above equation; at larger values of $r$, reflections due to boundaries interfere with the signal. Of interest is the fact that at still larger distances phase changes by more than $180^{\circ}$.

Jackson and Amer (1980) have attempted a theoretical analysis of the piezoelectric signal generated when a beam of modulated light is incident on the absorbing solid. For an illuminated surface, the front portion expands more than its rear. Bending of the sample, therefore, occurs to oppose the compression of the rear. It is this bending that is responsible for the piezoelectric signal. There are two terms that account for the signal. One is the in-plate displacement due to the average temperature $T_{0}$ and the second term is due to the sample buckling because of the temperature gradient. When the transducer is on the laser side of the sample, the avetage term and the buckling term add. When the transducer is away from the laser, the terms subtract. When the terms add, the signal is proportional to the absorption coefficient and saturates at $\beta l \cong 1 \cdot 5$. When the transducer is away from the beam and the terms subtract, the relation between absorption coefficient and the signal is complex. The signal eventually decreases as the transducer is moved away from the light source and could become more than $180^{\circ}$ out of phase. Jackson and Amer (1980) found good agreement between the experimental results and their theoretical predictions.

Farrow et al (1978), who used a chopped laser beam and a piezoelectric transducer for the detection of the photoacoustic signal from a Nd glass sample, found a phase shift in excess of $180^{\circ}$ in their signal when the laser beam was gently focussed compared to when it was focussed. The dependence of the phase on the radiation 
density was attributed to a distortion of the acoustic wave form by a ground state depletion. These workers argue that it is not possible for an acoustic transducer such as a gas microphone to monitor the mechanical vibrations of a solid (based on acoustic impedance matching arguments).

Tam and Patel (1979) have shown that a pulsed dye laser, a piezoelectric transducer directly coupled to the sample, and a gated PA detection technique, can yield sensitive, high-resolution spectra. They tested this method to obtain the weak visible absorption band of benzene near $607 \mathrm{~nm}$ due to the sixth harmonic of the $\mathrm{C}-\mathrm{H}$ stretch. The signal-to-noise ratio exceeded $10^{2}$ at the absorption peak. These authors have also considered the merits of chopped $\mathrm{cw}$ lasers and pulsed lasers. In the pulsed dye laser techniques the photoacoustic signal shows a ringing pattern due to the shock wave generated in the laser path through the medium. resonating in the photoacoustic cell. The sound velocity in the medium and the distance ketween the laser beam and the transducer determine the arrival time of the first peak. In pi inciple, one can, therefore, obtain the ultrasound velocity in the material and the value of $1.32 \pm 0.004 \times 10^{5} \mathrm{~cm} \mathrm{sec}^{-1}$ obtained by Tam and Patel (1979a) for the velocity of ultrasound in benzene agrees well with the known velocities.

For typical cell dimensions, acoustic resonances of the ringing pattern due to the passage of a laser beam are expected to be of the order of $10^{5} \mathrm{~Hz}$. The ambient noise problem is therefore reduced and the signal may be processed through a suitable high frequency band pass circuit without the use of lock-in amplifier. A boxcar was used to measure the hejght $H$ of the largest positive peak of the transient output. By using a ratiometer to divide $H$ by the pulse energy, one can obtain spectra at different wavelengths. Spectra of rare-earth oxide powders have

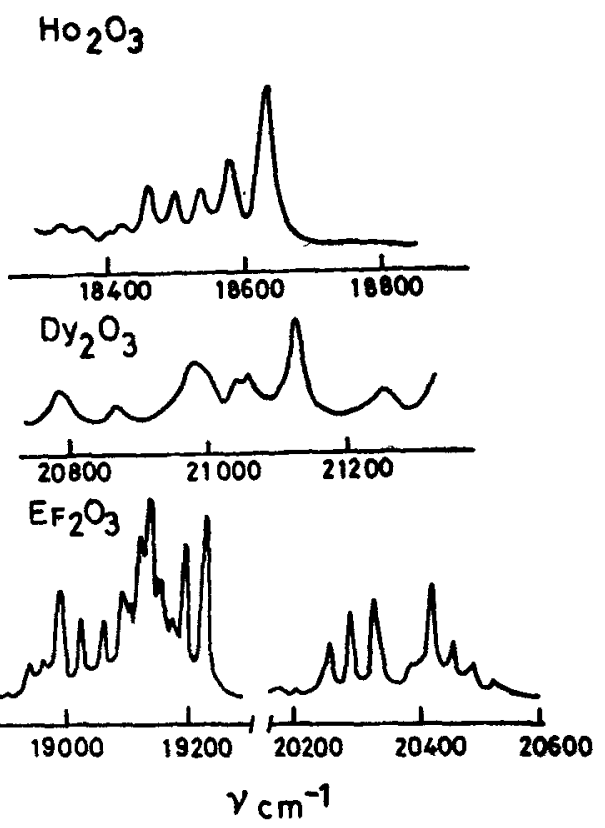

Figure 29. Piezoelectric PAS of some rare earth oxides (From Tam and Patel 1979b). 
been obtained by Tam and Patel (1979b) by this technique (figure 29). In order to avoid problems due to scattered light, an L-shaped quartz plate is used for transmitting the signal from the sample to the transducer. There is a resemblance between the transmission of the signal through the L-shaped quartz plate used in this technique and the stretched rubber memberne used by Bell to hear the signal with his ear a foot away from the light source.

Sam and Shand (1979) have developed a method analogous to that of Tam and Patel for the piezoelectric detection of PAS signal by immersing the sample in a liquid cell. The sample is suspended above a piezoelectric transducer and laser light strikes the surface of the sample away fi om the tiansducer. Since the velocity of the sound in the solid and the liquid are different, sound waves reaching the transducer due to the solid and the liquid may be well resolved especially if the thickness of the sample is made large enough. By processing the signal with boxcar averagers, the absorption spectrum of the bulk material may be obtained without interference from the optical properties of $j$ ts surface and the surrounding liquid.

Hadley et al (1980) have found that pulsed lightcan excite mechanical vibration modes of absorbing solid materials. The transfer efficiency for the conversicn of visible energy into the mechanical energy of vibrations is of the order of $10^{-9}$. They have proposed a thermal mechanism.

\section{Therma-optical spectroscopy}

Boccara et al (1980) have described a new experimental method in which they utilise the mirage effect. The periodic temperature gradient on the surface due to the absorption of the modulated light gives rise to a refractive index gradient which periodically deflects a probe beam parallel to the surface of the solid. The magnitude of the deflection is proportional to the energy absorbed and hence radiated. Thus, one can obtain an absorption coefficient dependent signal in all cases where photoacoustic signals do not saturate. These authors have utilised the tochnique for thermo-optical measurements below the $\lambda$ point of liquid helium. The hear standing wave (second sound) in the tail of the helium Dewar is created and the time dependent refractive index gradients thus generated. This technique has the same sensitivity as the photoacoustic method and has the additional advantage that it does not require closed cells. Murphy and Aamodt (1980) have developed a theory for thermo-optical spectroscopy which they term as optical beam deflection spectroscopy (OBDS) and consider it as one of the generic branches of photother mal spectroscopy (see also Bilmeyer et al 1977, 1980).

\section{Studies of surfaces}

Photoacoustic spectra should be free from saturation effecis when an absorbing system is present in a monolayer. Indeed most organic dyes which show saturation effects when spectra are recorded with bulk solids show true spectra when taken as thin films. In figure 30 we show the spectrum of a piece of filter-paper with a drop of methylene blue solution. The spectrum shows the expected features of methylene blue. From figure 30 we also see that blue paper or blue cloth shows bands characteristic of the colour. In figure 31 the spectrum of 


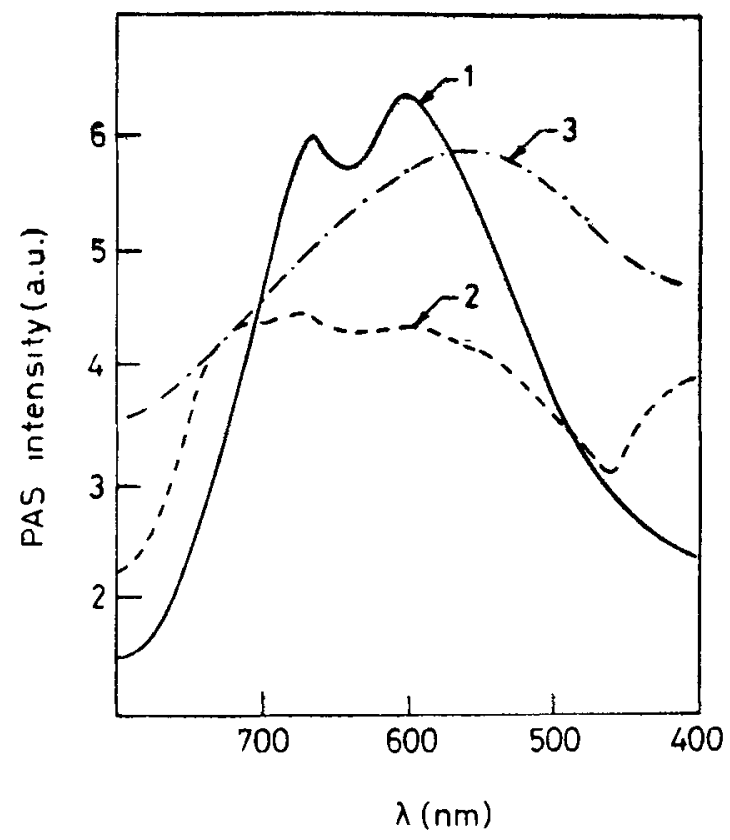

Figure 30. Normalized PAS from (1) methylene blue on filter-paper, (2) blue paper and (3) blue cloth.

Rhodamine B solution (0.0012 M) is shown. The sample holder has a depth of $2.2 \mathrm{~mm}$ and the chopping frequency was $23 \mathrm{~Hz}$. At this concentration of Rhodamine $B, \beta \approx 100 \mathrm{~cm}^{-1}$ and $1 / \beta<\mu_{\text {liquld }}$, where $\mu_{\text {liould }}$ is the thermal diffusion length in the sample; $1 / \beta$ is less than the sample thickness. We obtain a reasonably sharp spectrum with a maximum at 542 and a shoulder at $520 \mathrm{~nm}$. The intensity of the shoulder at $520 \mathrm{~nm}$ increases markedly when $\mathrm{BaCl}_{2}$ is added (to make $1 \mathrm{M}$ ) to the Rhodamine $B$ solution. The feature at $520 \mathrm{~nm}$ is quite sensitive to the envircnment. Rhodamine $B$ adsorbed as a monolayer on alumina or $\mathrm{GdAlO}_{3}$ shows differences in the band shape. Figure 32 shows the spectrum of methylene blue adsorbed on two different samples of alumina. We see marked differences in the spectrum on the two surfaces.

Absorption of dyes from dilute aqueous solutions does not generally give more than a monolayer coverage. It was of interest to us to examine the effect of the absorbed monolayer on the spectral features of the substrate. PAS of methylene blue adsorbed under monolayer conditions on $\mathrm{Nd}_{2} \mathrm{O}_{3}$ or $\mathrm{Ho}_{2} \mathrm{O}_{3}$ showed the features due to both methylene blue and $\mathrm{Nd}_{2} \mathrm{O}_{3}$ or $\mathrm{H}_{2} \mathrm{O}_{3}$. With Rhodamine $\mathrm{B}$, the features of the rare-earth oxides were almost submerged. It therefore seems that even a monolayer can markedly affect signals from the substrate in certain instances.

Fujihara et al (1978) have found a lower photoacoustic yield for Rhodamine B on $\mathrm{SnO}_{2}$ and $\mathrm{TiO}_{2}$. These results can be interpreted in terms of deactivation of the excited dyes by energy transfer to the conduction bands of the oxides and the reduction of the oxidized dyes by electron transfer. A mechenism involving a quenching process and re-reduction of oxidation products was proposed by Peltinger et al (1973) to explain the reduced sensitization current at highly doped $\mathrm{ZnO}$ electrodes. 


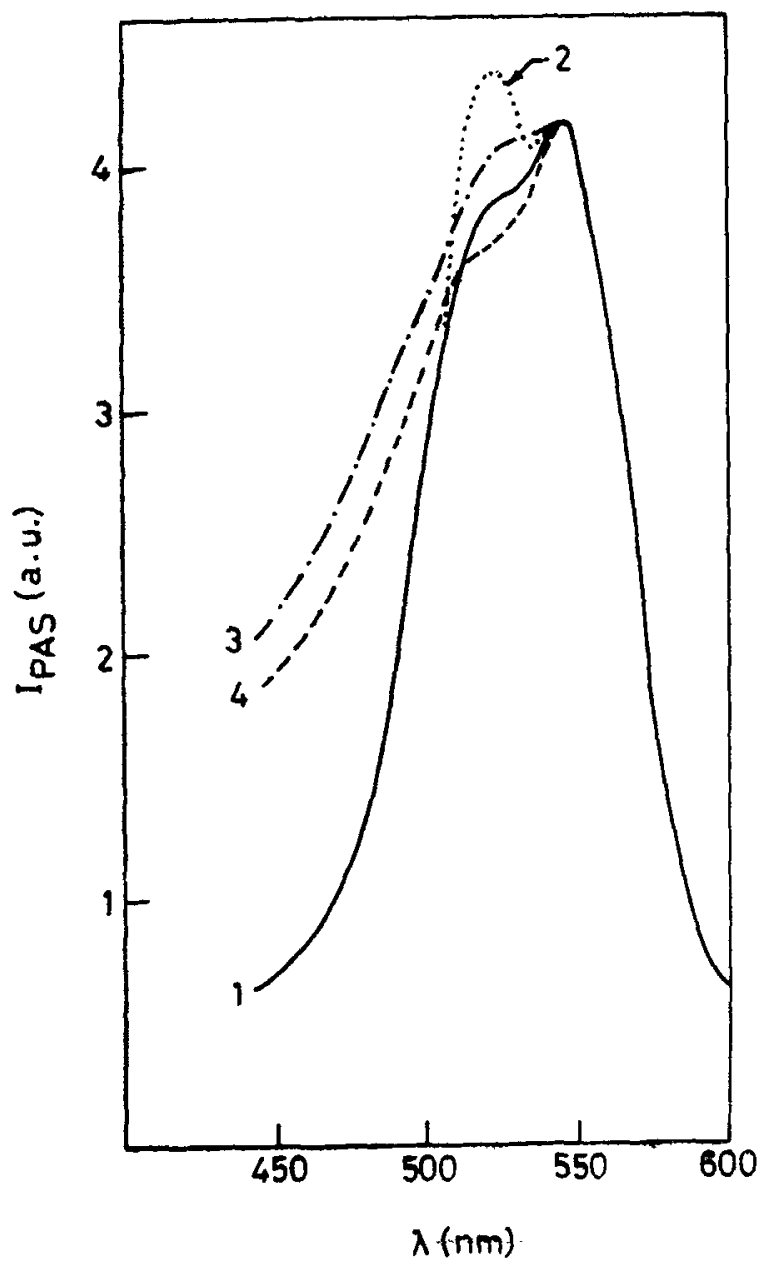

Figure 31. Normalized PAS of (1) Rhodamine B solution $0.0012 \mathrm{M}$, (2) Rhodamine $\mathrm{B}$ solution $\left(0.0012 \mathrm{M}+1 \mathrm{M} \mathrm{BaCl}_{2}\right)$, (3) Rhodaminc $\mathrm{B}$ on $\mathrm{Al}_{2} \mathrm{O}_{3}$ and (4) Rhodamine $\mathrm{B}$ on $\mathrm{GdAlO}_{\mathbf{8}}$.

Iwasaki et al (1980) have employed photoacoustic spectroscopy to investigate the photochemical reaction of sensitized dyes absorbed on semiconductor powders. 1, 1'-diethyl-2, 2'-cyanine and Rhodamine $\mathrm{B}$ adsorbed on $\mathrm{ZnO}$ from an aqueous solution were irradiated with light from a Xe lamp with wavelengths longer than $500 \mathrm{~nm}$ to eliminate the intrinsic absorption of the $\mathrm{ZnO}$ powder. With the 1, 1'diethyl-2, 2'-cyanine dye, the photoacoustic signal decreases with exposure to the excitation light without any appreciable shift in the spectrum which may be related to a rather drastic decomposition of the dye chromophores. With Rhodamine B, the maximum in the spectrum exhibited a gradual hypsochromic shift in the course of the exposure due to the occurrence of an effective $\mathrm{N}$-dealkylation of the dye. The rate of disappearance of the species responsible for the $510 \mathrm{~nm}$ band was negligibly small compared with the spectral shift from 565 to $510 \mathrm{~nm}$. The injected electron from the excited dye into the conduction band of $\mathrm{ZnO}$ must be subsequently removed by interaction with oxygen (or oxygen and water) to form $\mathrm{O}^{2-}$ which subsequently reacts with the electron acceptors. 


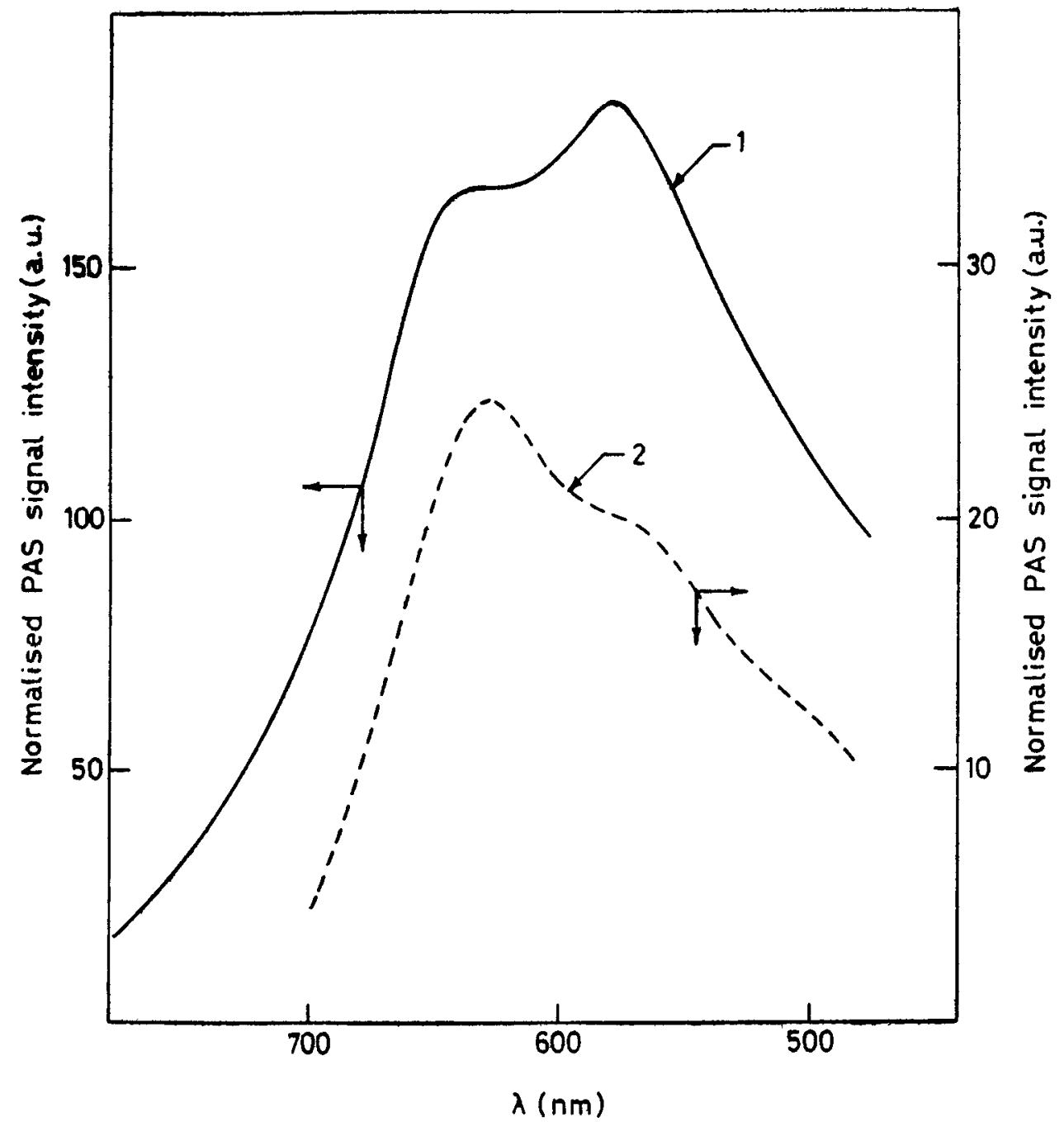

Figure 32. PA spectra of methylene blue adsorbed on (1) unactivated $\gamma$-alumina and on (2) commercial chromatographic alumina.

We saw in figure 32 that PAS band intensities of methylene blue adsorbed on two different aluminas were different. We have found that the intensity of the PAS bands of crystal violet adsorbed on a surface from solutions of different concentrations of the dye vary markedly. We have sought to exploit this feature to monitor surface areas of oxides. Photoacoustic spectra of dyes adsorbed on oxide surfaces could indeed provide a simple and fast method to determine relative surface areas of a large number of samples especially if the surface area of one of the samples is known. The study of surface areas carried out by us is outlined below.

Photoacoustic spectra of a commercial activated alumina and a BDH chromatographic alumina with adsorbed crystal violet were recorded for various initial concentrations of the dye (figure 33 ). Experimentally, $0.2 \mathrm{~g}$ samples of the solid were shaken in stoppered bottles with $5 \mathrm{ml}$ solutions of varying concentrations $\mathrm{cf}$ crystal violet for more than $1 \mathrm{hr}$. The solids were filtered and dried in a vacuum 


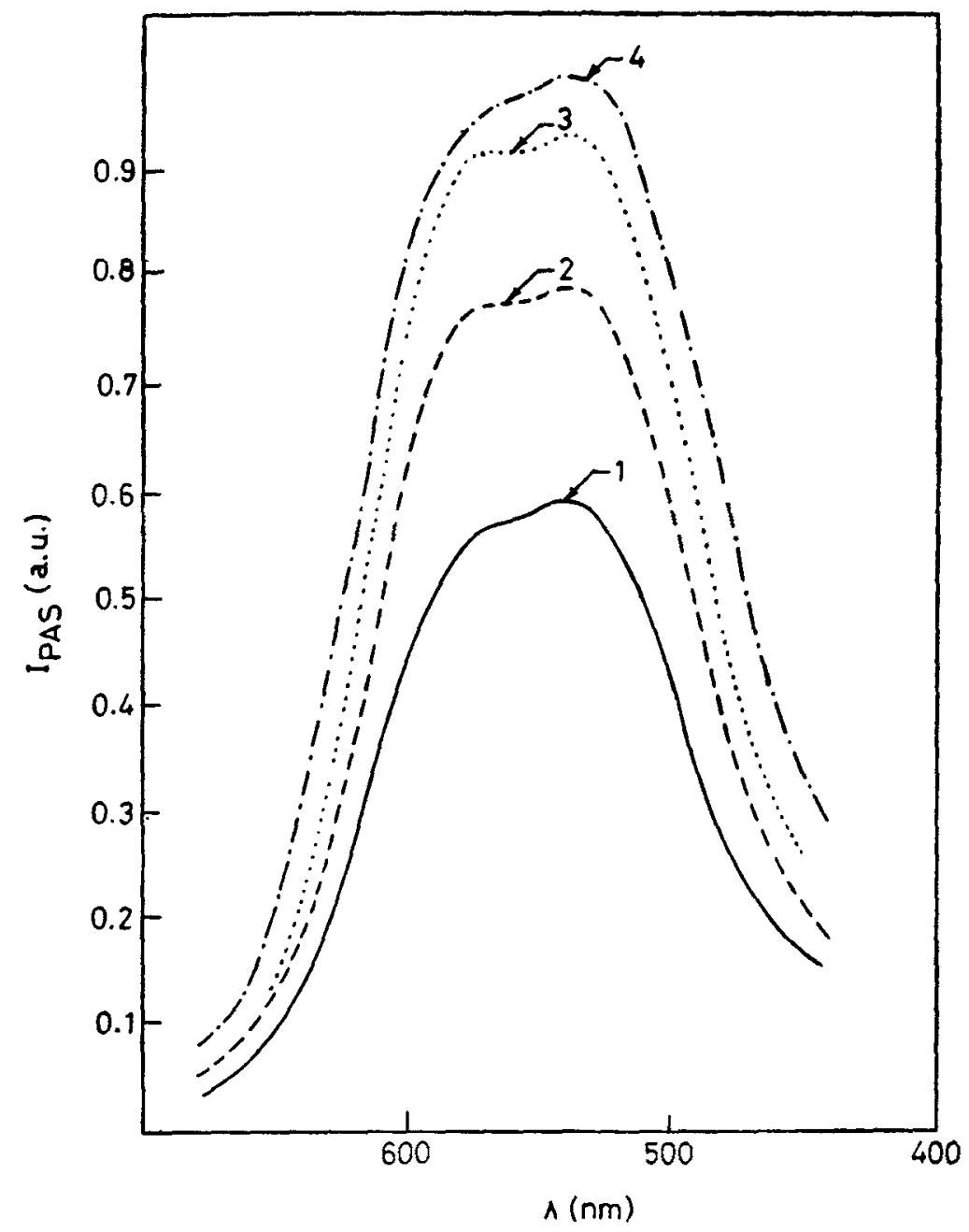

Figure 33. PAS of crystal violet lactone on alumina corresponding to various monolayer coverages : (1) 0.25 , (2) 0.36 , (3) 0.43 , (4) 0.45 .

dessicator before the spectra were taken. The photoacoustic band intensities at $580 \mathrm{~nm}$ (after normalization with respect to the power spectrum of the source) per unit weight of the sample shows an increase with the increase in the initial concentration of the dye and reaches a maximum value corresponding to complete coverage of the surface (figure 34 ). The ratio of the intensities at maximum coverage for the activated alumina and the BDH chromatographic alumina was $4 \cdot 7$.

In order to check whether the ratio of PAS intensities accords with the actua ratio of the surface areas, visible absorption spectroscopy was employed simultaneously to estimate the concentrations of solutions after equilibration with the solids. Thus, the decrease in the concentration after absorption $\triangle C$ and the equilibrium concentration $C$ could be fitted well into the Langmuir equation

$$
C / \Delta C=C / \triangle C_{\mathrm{max}}+1 / K \Delta C_{\mathrm{max}}
$$

From the plots of $C / \triangle C$ vs $C$, the amount adsorbed for complete monolayer coverage $\Delta C_{\mathrm{mex}}$, and hence the surface areas were calculated, using a value of 
$90 \AA^{2}$ for the cross-section of the crystal violet molecule (Giles et al 1964). The estimated surface of 179 and $39 \mathrm{~m}^{2} \mathrm{~g}^{-1}$ respectively for activated alumina and BDH chromatographic alumina are in the ratio $4 \cdot 6$, in agreement with the PAS intensity ratio. The photoacoustic signal intensity plotted as a function of the fraction covered (i.e. the ratio $C / \Delta C_{\max }$ ) shows a linear relation (figure 35). We observe that the lines pass through the origin and also that the slopes are in the ratio 4.7 for the two alumina samples. We have investigated if PAS could be effectively employed to determine surface acidities of catalysts using the $n$-butyl amine titration technique (Jagannathan et al 1981). Indicators such as bromothymol blue, bromocresol green and thymol blue show colour changes at two values of the acidity function $(-1 \cdot 5$ and $+6 \cdot 8,-3 \cdot 7$ and $+4 \cdot 6,+1 \cdot 7$ and $+8 \cdot 9$, respectively). Since the Hammett acidity function $H_{P}$ may be expressed in the form, $H_{0}=p K_{H B^{+}}$ $-\log C_{H B^{+}} / C_{B}$, where $\mathrm{HB}^{+}$and $\mathrm{B}$ are the conjugate acid and base forms respectively of the indicator, from the ratio of the PAS signal intensities of the two forms we

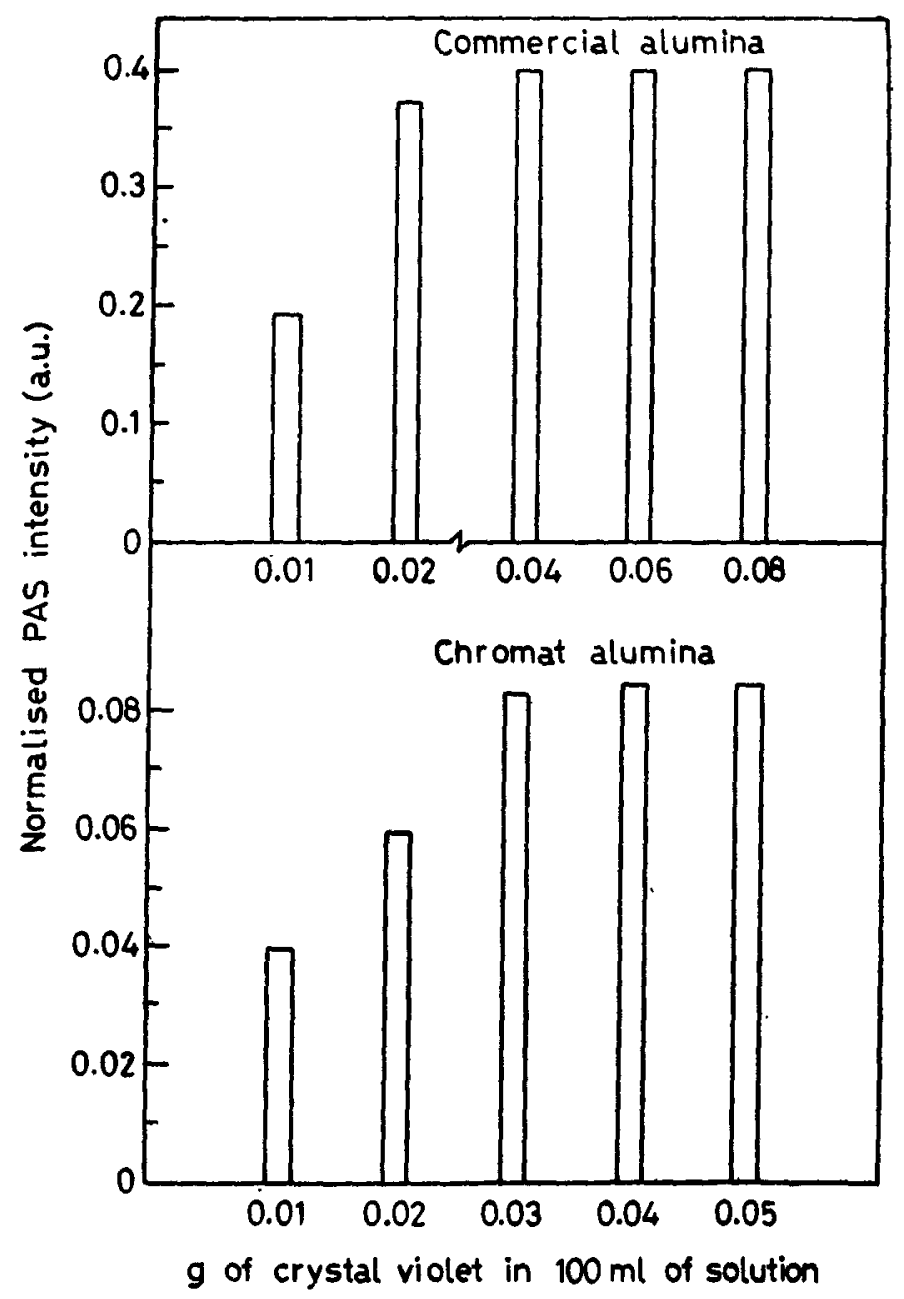

Figure 34. PA intensity vs. concentration of solution from which the crystal violet was adsorbed on the alumina surface. 


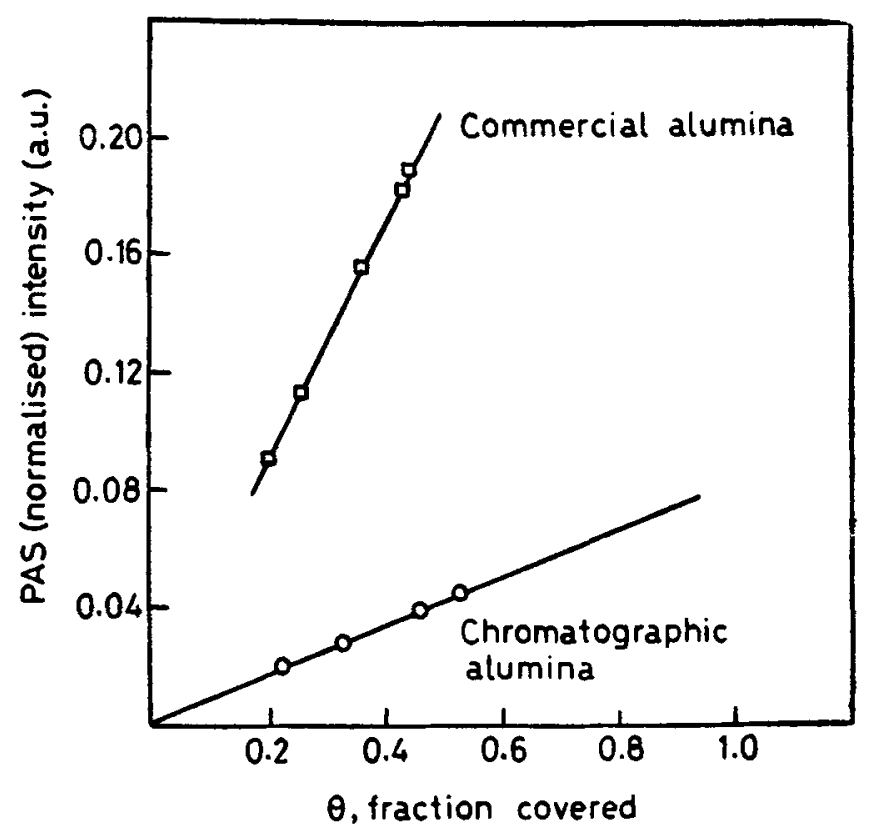

Figure 35. PA intensity vs. surface coverage on a commercial activated $\gamma$-alumina and BDH chromatographic alumina as a function of surface coverage. The ratio of the slopes $=4 \cdot 7$. The ratio of the surface areas is $4 \cdot 6$.

can directly obtain the $H_{0}$ value corresponding to every stage of the titration. The procedure followed by us is as follows.

Samples of a catalyst (about $0.2 \mathrm{~g}$ ) activated at $450-500^{\circ} \mathrm{C}$ were weighed and the necessary amount of the indicator solution in benzene added under perfectly dry conditions. A solution of $n$-butyl amine in benzene was added in varying amounts to the samples and equilibrated with intermittent shaking. The solid slurry was transferred to the cell of the photoacoustic spectrometer. All the operations were carried out in a dry glove box.

Typical spectra obtained with $\mathrm{H}_{2} \mathrm{SO}_{4} / \mathrm{SiO}_{2}$ employing bromocresol green are shown in figure 36 . Before the addition of $n$-butyl amine, two bands at $550 \mathrm{~nm}$ and $420 \mathrm{~nm}$ are observed. The $550 \mathrm{~nm}$ band corresponds to the acid form for the first titration range (lower $p K_{H_{B}}{ }^{\text {) }}$. In the second titration region, the band at $600 \mathrm{~nm}$ corresponds to the base form while the band at $420 \mathrm{~nm}$ corresponds to the acid form. Guided visually by the colour changes, it is easy to decide on the amounts of $n$-butyl amine to give $H_{0}$ values on either side of the $p K_{H B}+$ of a particular indicator.

By the above procedure, acidity distributions of several catalysts were obtained and the results are shown in figure 37. For silica and alumina samples the distributions correspond well with those reported by Druhsel and Sommers (1966).

We have also carried out a PAS study of alumina surfaces modified by $\mathrm{NaOH}$ using phenolphthalein as indicator. Using appropriate amounts of $\mathrm{NaOH}$ solution to impregnate the surface of alumina, samples containing $0.2,0.5,1.0$, $1 \cdot 5$ and $2 \cdot 0 \%$ (by weight) of $\mathrm{Na}_{2} \mathrm{O}$ on the surface after calcination were prepared. 


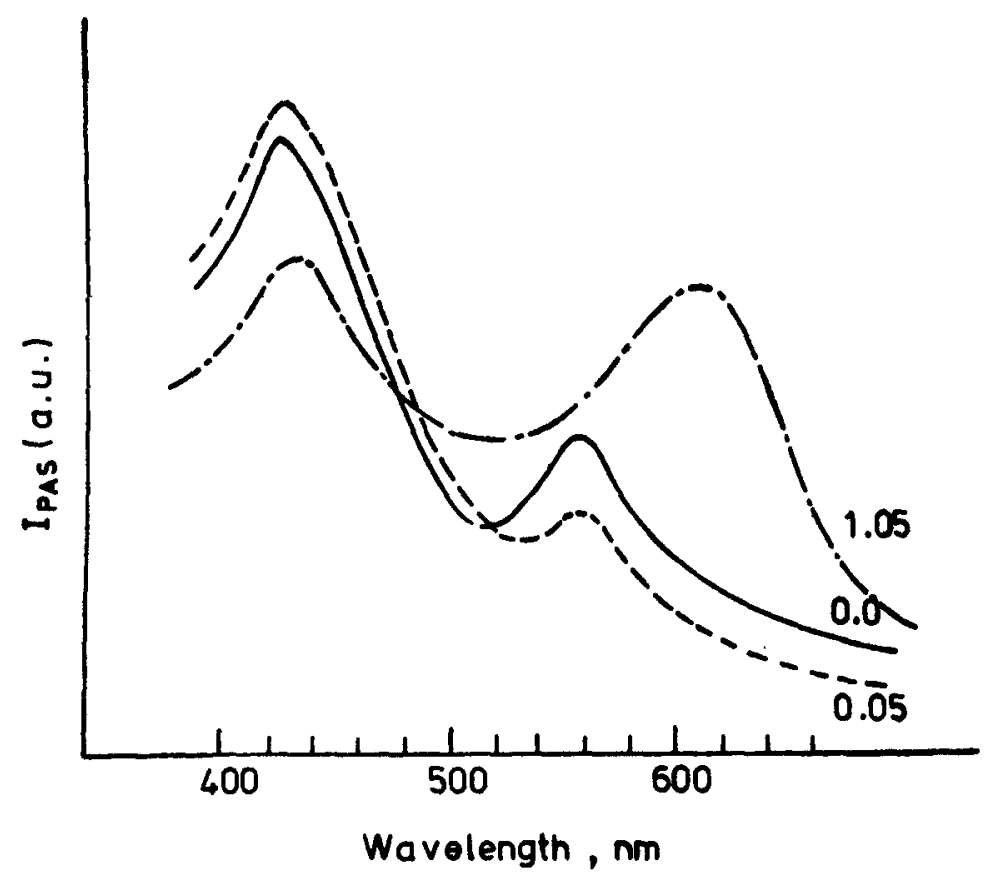

Figure 36. PAS of bromocresol green adsorbed on $\mathrm{H}_{2} \mathrm{SO}_{4} / \mathrm{SiO}_{2}$ as a function of the amount of $n$-butylamine (in millimole/g of solid).

Each of these samples was treated with phenolphthalein in dry ether in a dry glove box. Photoacoustic spectra obtained after drying the ether show band maxima at $540 \mathrm{~nm}$ corresponding to phenolphthalein in its basic form. The intensity of the $540 \mathrm{~nm}$ band varies with the amount of sodium oxide on the surface (figure 38). The intensity of the $540 \mathrm{~nm}$ band decreases in samples containing 0.2 and $0.5 \% \mathrm{Na}_{2} \mathrm{O}$ possibly due to the depletion of the highly basic coordinatively unsaturated $\mathrm{O}^{2-}$ ions associated with Lewis centres on the surface (Parry 1963). Replacement of the hydrogen of the surface hydroxyls which are mostly nonacidic (Parry 1963 ; Peri 1965) by sodium ions enhance the basicity of the surface and this accounts for the enhanced intensity of the $540 \mathrm{~nm}$ band in samples containing $1 \%$ or more $\mathrm{Na}_{2} \mathrm{O}$.

PAS can be a useful tool in the study of derivatized surfaces. Lochmuller et al (1980) have examined the PAS of chemically modified powder surfaces. This is exemplified by bonded charge-transfer chromatographic stationary phases which employ organic moieties immobilized on a microparticulate gel. An electron deficient aromatic molecule such as 1-fluoro-2, 4-dinitrobenzene (DNP) is made to react with an activated silica surface according to the reaction sequence given below :

Aminated silica

$$
: \mathrm{Si}-\mathrm{OH}+(\mathrm{EtO})_{3} \mathrm{Si}\left(\mathrm{CH}_{2}\right)_{3} \mathrm{NH}_{2} \rightarrow: \mathrm{Si}-\mathrm{O}-\mathrm{Si}\left(\mathrm{CH}_{2}\right)_{3} \mathrm{NH}_{2}
$$




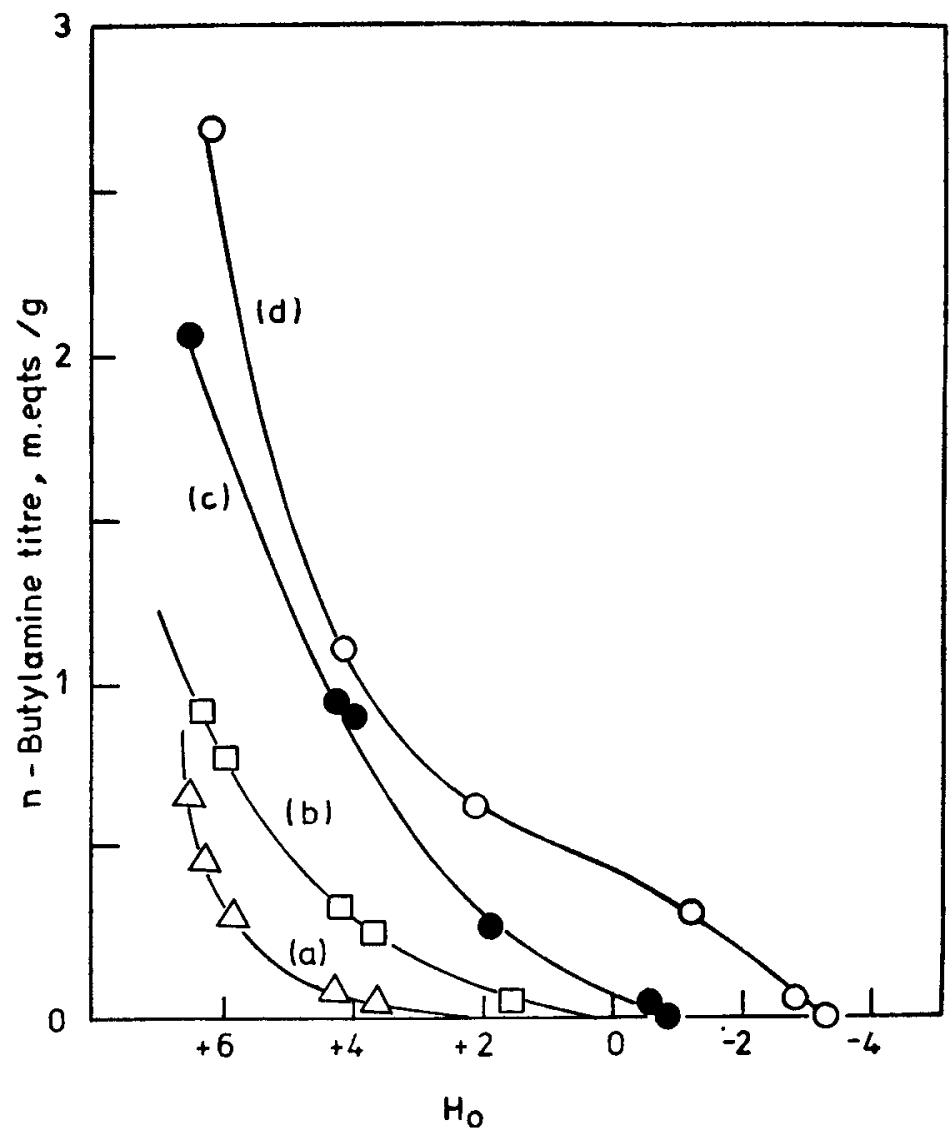

Figure 37. Plot of the $n$-butylamine titre against the Hammett acidity $H_{0}$ for (a) silica (b) alumina (c) molecular sieve $13 \mathrm{X}$. (d) $\mathrm{H}_{2} \mathrm{SO}_{4} / \mathrm{SiO}_{2}$.

DNP phase

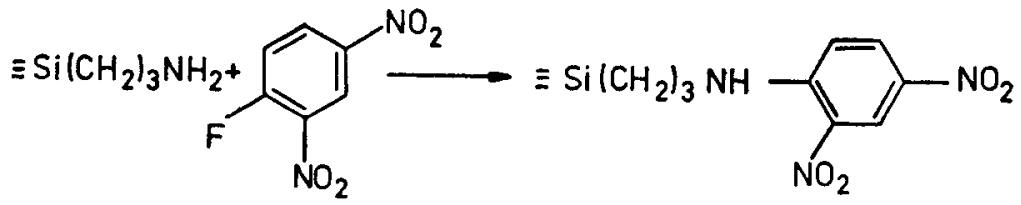

In this way the electron deficient moiety is insulated from the silica surface by connecting alkyl chain and presumably is allowed sufficient mobility to maximize interaction with the solute molecules in the chromatographic mobile phase. The spectra of the DNP bonded phase and the model compound 2,4-dinitro-N-butylaniline (DNBA) (in the solid and in the solution phase) showed that the DNP bonded phase exhibits a spectrum that is virtually the same as the solid model compound. This signifies a derivative gel scheme which has successfully immo- 


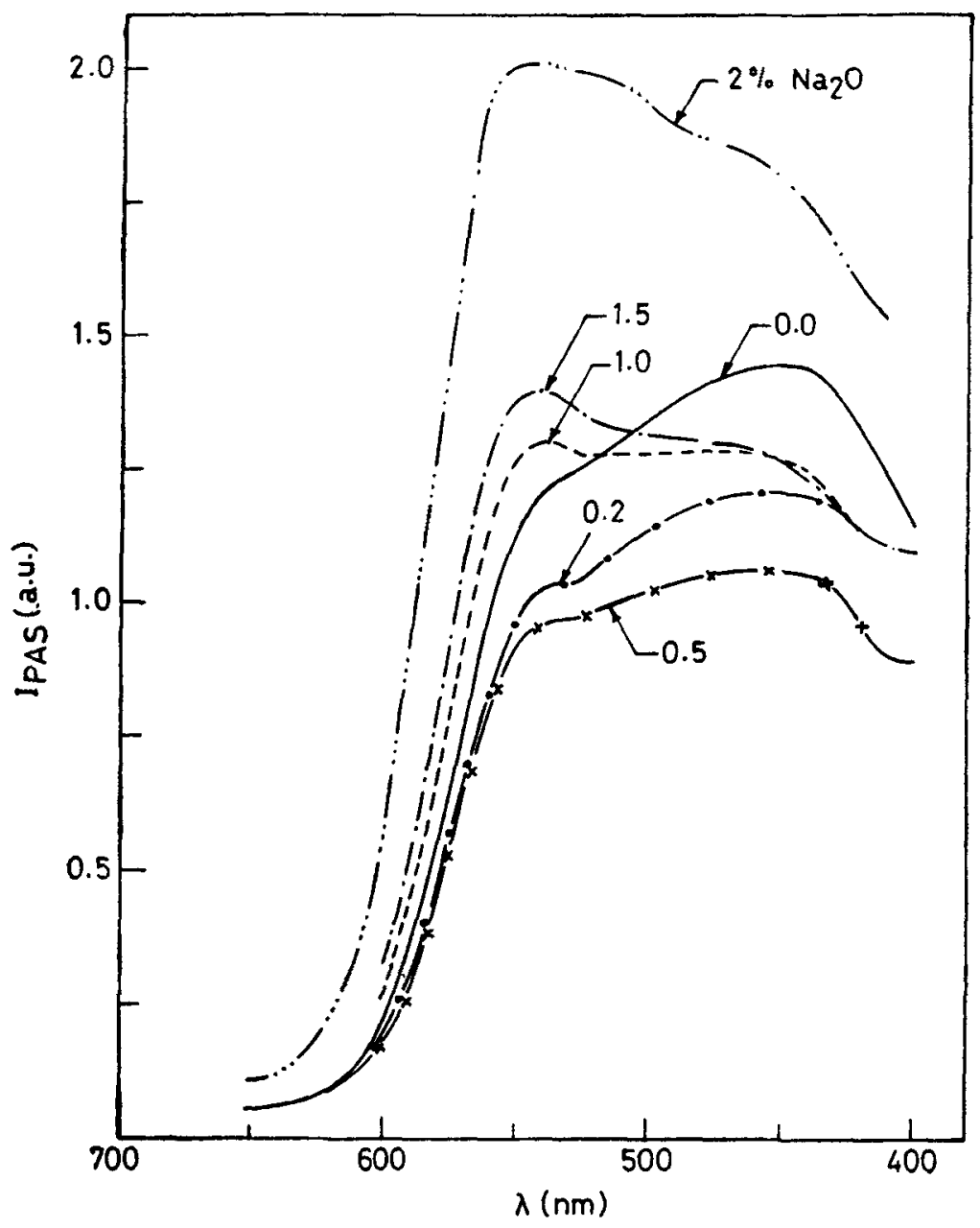

Figure 38. Variation of the PA intensity of the $540 \mathrm{~nm}$ band in the photoacoustic spectra of adsorbed phenolphthalein with sodium content of the alumina surface.

bilized the chromophore without altering its spectral properties. When the DNP was immobilized using a linear aminopropyl silicone, the absorption band shifted from 350 to $342 \mathrm{~nm}$. The aminopropyl silicone is polymerized on the surface in the presence of trace amounts of water. The subsequent reaction with DNP had evidently produced a phase whereby the charge transfer moiety was attached to the support material by both direct alkyl linkage to the surface and also by linkages to the silicone polymer. Lochmuller et al (1980) have shown that the bonded phase of 2-quinazoline has its absorption features markedly changed by treatment of the surface with aqueous solutions at various $\mathrm{pH}$ values. The change is a consequence of the alteration of the chromophore through protonation and to some extent covalent hydration. In this laboratory, we have been able to obtain the spectra of charge transfer complexes and aromatic radical ions on oxide surfaces, 
PAS would be useful for the study of metal states on surfaces as distinct from those in the bulk. In figure $39 \mathrm{a}$, we show the spectra of an alumina sample impregnated with $10 \%$ nickel from a nickel nitrate solution. The impregnated alumina samples were heated at $110^{\circ}, 400^{\circ}, 600^{\circ}$ and $800^{\circ} \mathrm{C}$ for 24 hours. The sample heated at $110^{\circ} \mathrm{C}$ shows a spectrum resembling that of $\mathrm{NiO}$ or $\mathrm{KNiF}_{3}$ and probably shows $\mathrm{Ni}$ in octahedral coordination. On heating further, a band around $580 \mathrm{~nm}$ develops rapidly. We believe that this new band is due to nickel that may form clusters or diffuse into the bulk of the alumina support. In figure $39 \mathrm{~b}, \mathrm{PAS}$ of dimethylglyoxime adsorbed on catalysts from a saturated ammoniacal solution is shown. The intensity of the dimethylglyoxime complex band is more than halved in the sample heated at $400^{\circ} \mathrm{C}$ compared to that heated at $100^{\circ} \mathrm{C}$. This shows that during heating, the nickel atoms have reorganised themselves into clusters
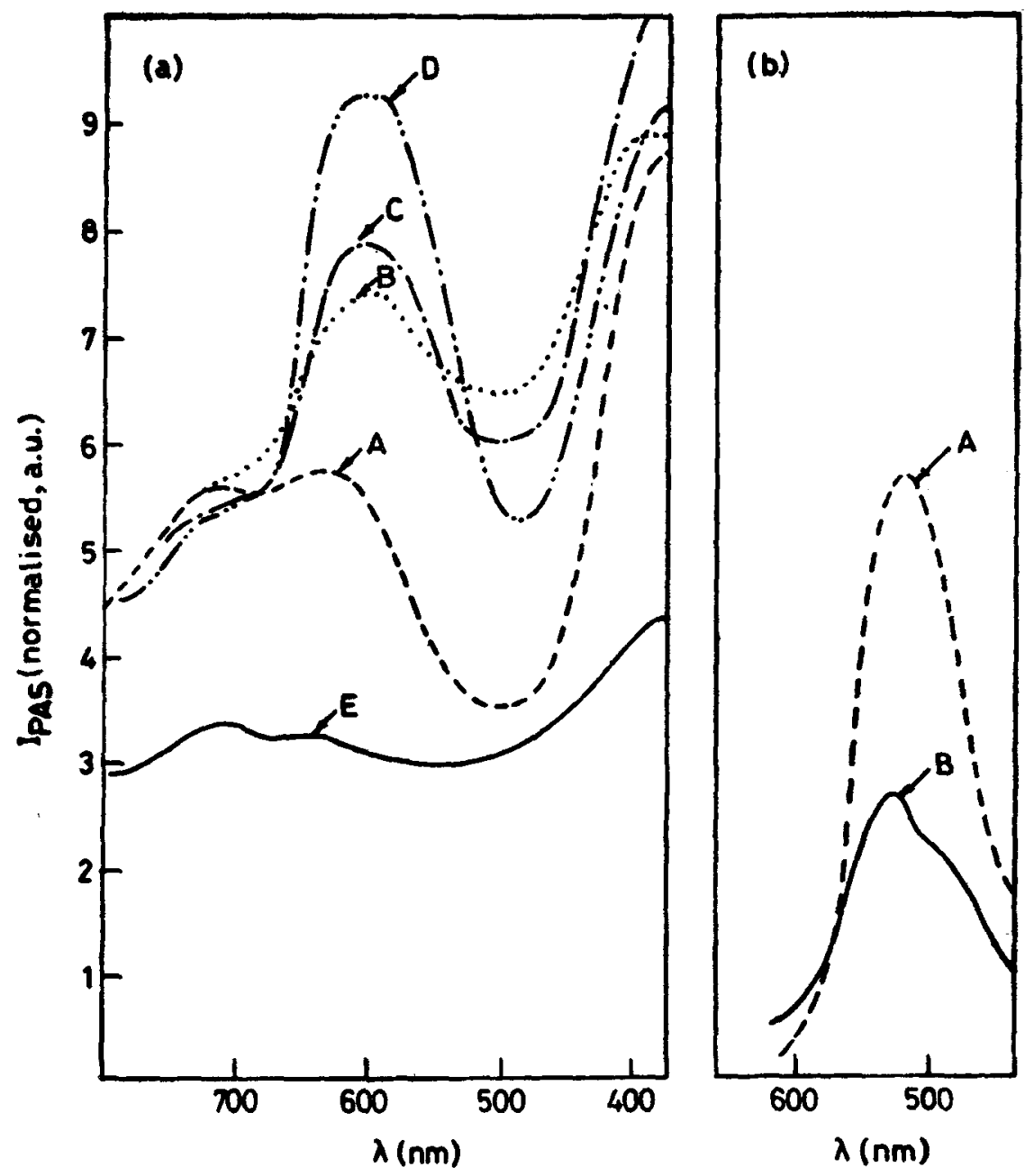

Figure 39. (a) $\mathrm{PAS}$ of $10 \% \mathrm{NiO}-\mathrm{Al}_{2} \mathrm{O}_{3}$ heated at (A) $110^{\circ} \mathrm{C}$, (B) $400^{\circ} \mathrm{C}$, (C) $600^{\circ} \mathrm{C}$ and (D) $800^{\circ} \mathrm{C}$. Spectrum of NiO is given by E. (b) PAS of the Nidimethylglyoxime complex formed on $\mathrm{NiO}-\mathrm{Al}_{2} \mathrm{O}_{3}$ surface heated at (A) $110^{\circ} \mathrm{C}$ and (B) $400^{\circ} \mathrm{C}$. 
or diffused into the bulk since the dimethylglyoxime would only complex with those nickel atoms on the surface. Very small amounts of nickel on surfaces (of the order of $10^{-5} \mathrm{~g}$ atom) can be detected by this technique.

Rosencwaig (1976) has pointed out the applicability of the PAS technique for obtaining spectra of TLC plates in situ. Castleden et al (1979) have examined the feasibility of PAS for the direct examination of TLC plates. Aluminium-foilbacked TLC plates used for chromatography were cut in $20 \mathrm{~mm}$ diameter discs. Discs with the analyte spot at its centre were placed directly in the cell. Fluorescein was spotted on the TLC plate and the development of the chromatogram was carried out in a solvent mixture containing ethylacetate $(33 \%)$ in acetone. After development, the chromatogram was allowed to dry in a desiccator. With sufficient practice, a linear calibration plot could be obtained for the determination of fluorescein in the range $0 \cdot 2-2 \mu \mathrm{g}$.

Low and Parodi (1980) have constructed a PA spectrometer for use in the far IR for the study of catalyst surfaces. Samples could be degassed and exposed to gases under controlled conditions in this design. The sample is treated at high temperatures and moved by a magnet through the bore of a stopcock into the microphone region. The sample holder was then pressed against the walls of the microphone region under the window by operating a Teflon screw. These authors have been able to see the characteristics of $-\mathrm{OH}$ species on surfaces and the effect of $\mathrm{NH}_{3}$ and other vapours. Rockley (1979) and Farrow et al (1979) have demonstrated the possibility of carrying out Fourier transform photoacoustic spectroscopy and this should be immensely useful for the study of surfaces.

\section{Depth profiling and subsurface imaging}

Since the light absorbed by a solid generates heat at various depths, only a fraction of the heat reaches the heat transfer surface of the sample during the duty cycle of the chopper, except at very low frequencies. Similarly, the heat generated by a chromophore separated from the surface by some non-absorbing material would reach the surface after a time determined by the thermal properties of the layer and the depth at which the chromophore is located. Hence, the frequency of the chopped light and the phase angle (with respect to a reference) at which the signal is maximum should be able to give valuable information of the depth and other structural features. The time lag $\triangle t$ in the PAS signal from a source at depth $x$ below a transparent layer is related to the period $p$ of the chopping cycle and the thermal diffusivity by the relationship,

$$
\Delta t=(x / 2)[p / \pi d]^{1 / 2} \text {. }
$$

A plot of $\Delta t$ (determined from phase angle differences) vs $p^{1 / 2}$ should then give a straight line. Adams and Kirkbright (1977) have obtained such straight line plots and have calculated the thermal diffusivity of materials from the slopes of these lines.

A simple demonstration of the usefulness of PAS for the analysis of two layers has been made by Adams et al (1976b) and King and Kirkbright (1976). Figure 40 shows the uncorrected spectrum of spinach leaf with the surface layer removed. The phase of the signal $\phi$ was adjusted to be maximum at $6.50 \mathrm{~nm}$. The spectrum 


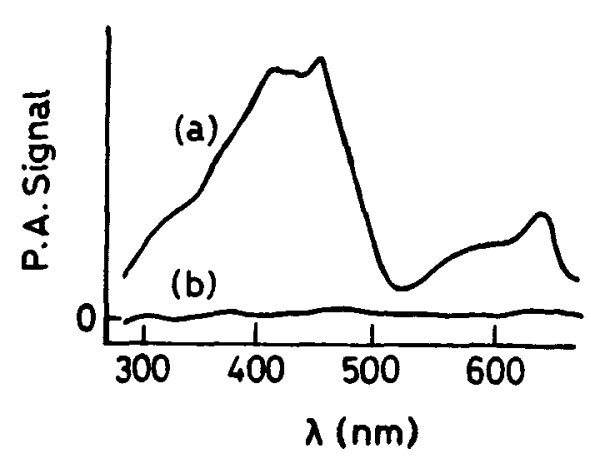

Figure 40. Uncorrected PAS of spinach leaf with wax cuticle layer removed : (a) at a phase $\phi$ and (b) at $\phi-90^{\circ}$ (From King and Kirkbright 1976).

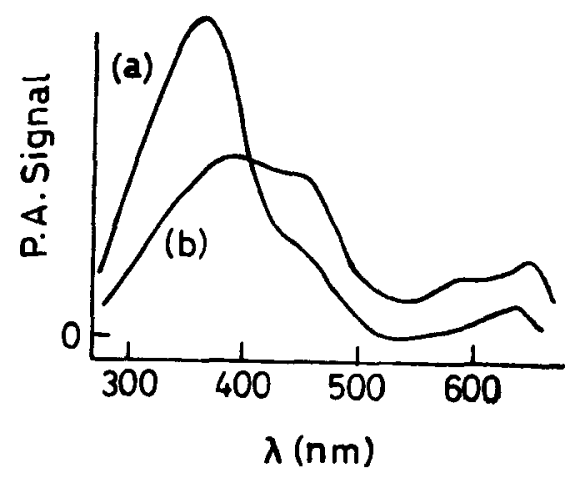

Figure 41. Uncorrected PAS of intact spinach leaf (a) at the same phase $\phi$ as in Fig. 40 and (b) at $\phi-90^{\circ}$. (From King and Kirkbright 1976).

corresponds to that of uncorrected chlorophyll. The $\left(\phi-90^{\circ}\right)$ phase signal as expected shows no phase dependence and is zero at all wavelengths. Figure 41 shows the uncorrected spectrum of the intact spinach leaf at the same phase angles $\phi$ and $\phi-90$ that was used for the experiments reported in figure 40 . At $\phi$ the spectrum of only the cuticle layer is seen, whereas the $\phi-90$ spectrum shows the features of chlorophyll.

From (23) we see that if $a$ is known, the depth $x$ may be calculated. This, in our opinion, would be of value in the study of membranes and other biological systems especially in transport studies with the aid of appropriate probes. It is therefore worthwhile to look at the cumulative PAS signal from two different sources at different depths or at different phases.

If the sample side of the cell window is blackened with a dilute solution of black ink, the PA signal shows a wavelength dependence similar to the power spectrum. If the phase of the signal is adjusted to be maximum at $800 \mathrm{~nm}$ and a green leaf introduced, the PA signal changes from a positive value at $800 \mathrm{~nm}$ to a negative value around $680 \mathrm{~nm}$ corresponding to a maximum of the chlorophyll band (figure 42). The phase of the signal changes by more than $100^{\circ}$. The quadrature component showed features of the chlorophyll spectrum similar to the $\phi$ PAS spectrum (figure 42). The crushed leaf gives features due to both the chlorophyll and the wax layer. These results show that (i) the phase shift can be more than $90^{\circ}$, (ii) interference between signals of different phases may occur and (iii) carbon black may be used as an internal reference in determining the phase shift.

Using the same blackened window, we have looked at the PA signal from an intact croton leaf which was prominently green on one side (the top) and red on the other side. The signal was maximised with respect to the phase at $800 \mathrm{~nm}$ and spectra taken at two different chopping frequencies of $17 \cdot 6$ and $35 \cdot 2 \mathrm{~Hz}$. The results (figure 43) indicate that signal due to the red part is almost at the same phase as the carbon black while the chlorophyll signal is about $90^{\circ}$ out of phase. This 


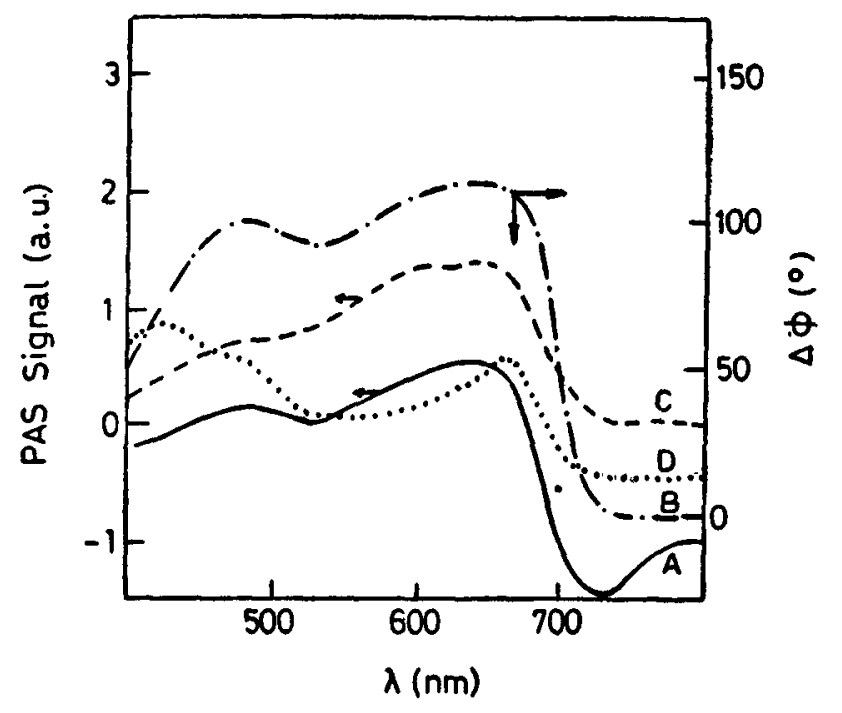

Figure 42. PAS signal from an intact green leaf (A) uncorrected signal from leaf with the phase adjusted for maximum at $800 \mathrm{~nm}$ and the window slightly blackened. (B) Change in the phase angle for the leaf. (C) The quadrature component for the signal A. (D) Spectrum of crushed leaf (scale not shown).

suggests that the chromophore responsible for the red colour are on the surface while the chromophores responsible for the green colour are under some nonabsorbing layer, probably the wax layer.

Adams et al (1978) have compared the spectra obtained from uncoated glass substrates and glass substrates coated with titanium dioxide and also with alternate layers of titanium dioxide and silicon dioxide. For the uncoated glass substrate, the reflectance spectrum shows a high specular component with very little visible detail. In the case of $\mathrm{TiO}_{2}$ coated glass substrates, the transmission and reflectance spectra besides showing the ultraviolet absorption band just as the PA spectra, also show the interference pattern at longer wavelengths. The spectra of dielectric coated laser mirrors also show the complementary nature of the reflection, transmission and PAS spectra. When the uncoated rear surface is illuminated, only PAS gives the spectrum of the glass substrate.

There has been considerable interest in employing photoacoustics for subsurface imaging and microscopy of solid surfaces. Wickramsinghe et al (1978) have demonstrated the feasibility of what they termed a "photoacoustic micro. scope" by modifying the input lens of a transmission acoustic microscope with an optical lens. Nanosecond laser pulses were made to impinge on the sample and the acoustic transducer in the microscope picked up the variations in sound as the object was scanned by the laser beam. The amplitude at various points is used to form the image. Wong et al (1978) have reported the detection of microcracks of ceramic $\mathrm{Si}_{3} \mathrm{~N}_{4}$ material through scanning photoacoustic detection techniques. These cracks are not usually detected under a microscope. Wong et al (1979) have also given experimental evidence for the non-destructive detection of subsurface structure of solids by a technique they term as scanning photo- 


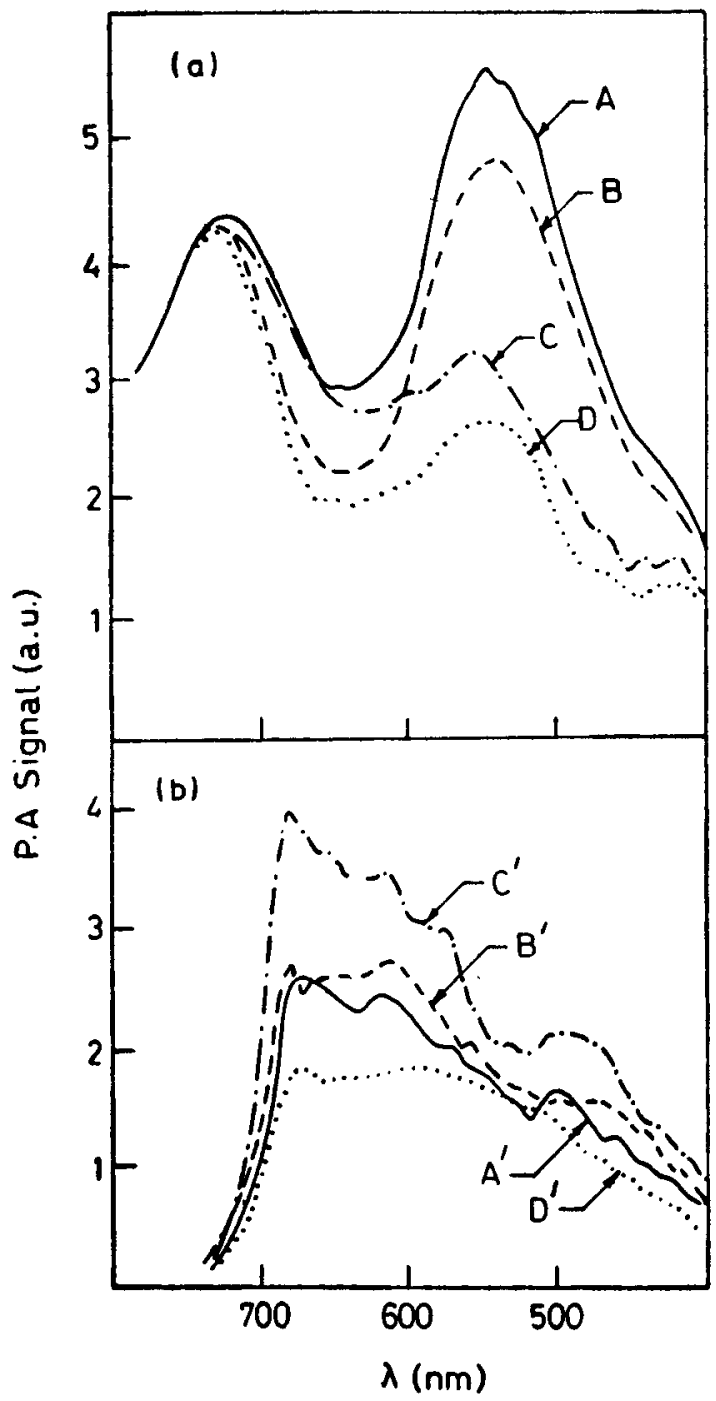

Figure 43. Uncorrected PA signal from a croton leaf with the window slightly blackened. (a) phase $(\phi)$ is maximum at $800 \mathrm{~nm}$ and (b) $\phi-90^{\circ}$. The signals were normalized for equal intensity and the phase $\phi$ adjusted for maximum signal at $800 \mathrm{~nm}$. (B, $\left.\mathrm{B}^{\prime}\right)$ and $\left(\mathrm{D}, \mathrm{D}^{\prime}\right), \omega=35.2 \mathrm{~Hz} ;\left(A, A^{\prime}\right)$ and $\left(C, C^{\prime}\right), \omega=17.6 \mathrm{~Hz}$; $\left(C, C^{\prime}\right)$ and $\left(D, D^{\prime}\right)$ green side; $\left(A, A^{\prime}\right)$ and $\left(B, B^{\prime}\right)$ rcd side.

acoustic microscopy (SPAM). The thickness of the thermal layer probed by this technique is of the arder of the thermal diffusion length which in the case of samples such as $\mathrm{Si}_{3} \mathrm{~N}_{4}$ and $\mathrm{SiC}$ is of the order of $100 \mu \mathrm{m}$ at $100 \mathrm{~Hz}$ chopping frequency. Busse (1979) points out that photoacoustic phase angle scanning (rather than amplitude scanning) is more effective in subsurface studies. Phase changes show up clearly the presence of subsurface holes which is not seen by the amplitude changes. There is no significant change in the phase when these holes are subsequently filled with the same metal perhaps due to the persistence of some 


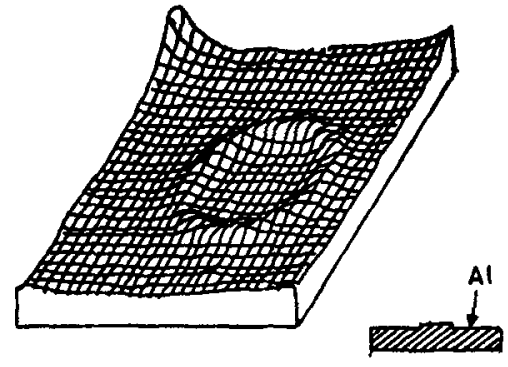

(a)

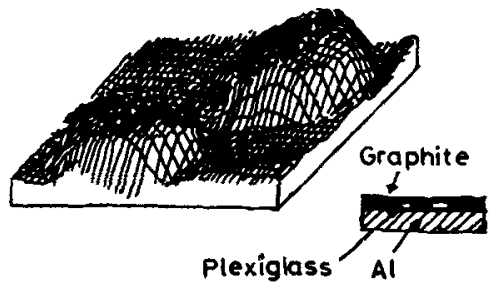

(b)

Figure 44. Photoacoustic imaging (a) image of an aluminium plate with a cylindrical projection; (b) Subsurface image of two plexiglass discs sandwiched between graphite and aluminium plates. (From Busse and Orgabeck, 1980)

thermal boundary. In this last case, photoacoustic imaging could be superior to $x$-ray imaging. A striking example of imaging that can be obtained is shown in figure 44.

Subsurface imaging resolution depends on the wavelength of the highly damped thermal wave in the sample and equals the thermal diffusion length. In gas microphone detection systems, the limit of the resolution is $10-20 \mu$ at best. Frequencies in the megahertz ranges are readily attainable with piezoelectric detection systems permitting resolution of $1 \mu$. Rosencwaig and Busse (1980) have used piezoelectric detection to obtain images which are neither optical images (i.e. limited by optical contrast) nor acoustic images but rather the ther mal wave images (Rosencwaig 1980) that arise from the interaction of the thermal waves with the subsurface holes.

\section{Acknowledgement}

The authors thank the Indian National Science Academy for support of this research and Dr K Jagannathan for assistance with some of the measurements.

\section{References}

Aamodt J C, Murphy L C and Parker J G 1977 J. Appl. Phys. 48927

Adams M J, Beadle B C and Kirkbright G F 1976 Analyst (London) 101553

Adams M J, Beadle B C and Kirkbright G F 1977 Analyst (London) 102569

Adams M J, Beadle B C and Kirkbright G F 1978 Anal. Chem. 501371

Adams M J, Beadle B C, Kirkbright G F and Menon K R 1978 Appl. Spectrosc. 32430

Adams M J, King A A and Kirkbright G F 1976 Analyst (London) 10173

Adams M J and Kirkbright G F 1977 Analyst (London) 102281

Bell A G 1881 Philos. Mag. 11510

Bennett H S and Forman R A 1976 Appl. Opt. 152405

Boccara A C, Fournier D and Badoz J 1980 Appl. Phys. Lett. 36130

Brilmeyer G H and Bard A J 1980 Anal. Chem. 52685 
Brilmeyer G H, Fujishima A, Santhane K V and Bard A J 1977 Anal. Chem. 492057

Busse G 1979 Appl. Phys. Lett. 35759

Busse G and Orgabeck A 1980 J. Appl. Phys. 513576

Castleden S L, Elliott C M, Kirkbright G F and Spillane D E M 1979 Anal. Chem. 51151

Cesar C L, Vargas H, Meyer J A and Miranda L C M 1979 Phys. Rev. Lett. 421570

Crowley T P, Faxvog F R and Roessler D M 1980 Appl. Phys. Lett. 36641

Druhsel H V and Sommers A L 1966 Anal. Chem. 381723

Ducharme D, Tessier A and Leblanc R M 1979 Rev. Sci. Instrum. 501461

Eaton H E and Stuart J D 1978 Anal. Chem. 50587

Evora C, Landers R and Vargas H 1980 Appl. Phys. Lett. 36864

Farrow M M, Burnham R K, Auzannean, Olsen S L, Purdie N and Eyring E M 1978 Appl. Opt. 171093

Farrow M M, Burnham R K and Eyring E M 1979 Adv. Study, Inst. Ser. C. C50 (Tech.) 161-174

Fernelius N C 1979 Appl. Opt. 181784

Ferrell W G and Haven Y 1977 J. Appl. Phys. 483984

Florian R, Pelzl J, Rosenberg M, Vargas H and Wernhardt R 1978 Phys. Stat. Sol. (a) 48 K35

Fournier D, Boccara A C and Badoz J 1978 Appl. Phys. Lett. 32640

Freeman J J, Friedman R M and Reihard H S 1980 J. Phys. Chem. 84315

Fujihara M, Osa T, Hursh D and Kuwara T 1978 J. Electroanal. Chem. 88285

Giles C H, Easton I A and McKay R B 1964 J. Chem. Soc. 4495

Gorelik G 1946 Dokl. Akad. Nauk SSSR 54779

Hadley J W, Lewis A V, Alexandroux C, Schwab C and Boatner L A 1980 Appl. Phys. Lett. 36736

Harshbarger W R and Robin M B 1973 Acc. Chem. Res. 6329

Hershberger W D, Bush E T and Leek G W 1946 RCA Rev. 7422

Hordvik A and Schlossberg H 1977 Appl. Opt. 16101

Iwasaki T, Oda S and Honda K 1980 J. Phys. Chem. 841060

Jackson W and Amer N M 1980 J. Appl. Phys. 513343

Jagannathan K, Ganguly P and Rao C N R $1981 \mathrm{~J}$. Catal. (under publication)

Kaiser R 1959 Can. J. Phys. 371499

Kanstad S O and Nordal P E 1978 Opt. Commun. 26367

Kerr E L 1973 Appl. Opt. 122520

King A A and Kirkbright G F 1976 Lab. Practice 25377

Korpium P, Baumann J, Luescher E, Papamokos E and Tilgner R 1980 Phys. Stat. Sol. (a) $58 \mathrm{~K} 13$.

Kreuzer L B 1971 J. Appl. Phys. 422934

Kreuzer L B and Patel C K N 1971 Science 17345

Kreuzer L B and Patel C K N 1972 Science 179349

Lin J W and Dudek L P 1979 Anal. Chem. 511627

Lochmuller C H, Marshall S F and Wilder D R 1980 Anal. Chem. 5219

Low M J D and Parodi G A 1980a Spectrosc. Lett. 13151

Low M J D and Parodi G A 1980b J. Mol. Struct. 61119

Low M J D and Parodi G A 1980e Appl. Spectrosc. 3476

McClelland J F and Kniseley R N 1976a Appl. Opt. 152658

McClelland J F and Kniseley R N 1976b Appl. Opt. 152967

McClelland J F and Kniseley R N 1979 Appl. Phys. Lett. 35585

McDonald F A 1980 Appl. Phys. Lett. 36123

McDonald F A and Wetsel G C 1978 J. Appl. Phys. 492313

Melamed N T 1963 J. Appl. Phys. 34560

Merkle L D and. Powell R C 1977 Chem. Phys. Lett. 46303

Merkle L D and Powell R C 1978 J. Phys. C11 3103

Michaels J E 1961 Planet. Space Sci. 7427

Monahan Jr, E M and Nolle A W 1977 J. Appl. Phys. 483519

Munroe D M and Reichard H S 1977 Am. Lab. 9 (2) 23

Murphy J C and Aamodt L C 1977a Appl. Phys. Lett. 31728

Murphy J C and Aamodt L C 1979b J. Appl. Phys. 483502 
Murphy J C and Aamodt L C 1980 J. Appl. Phys. 514580

Nunes O A C, Monteiro A M M and Neto K S 1979 Appl. Phys. Lett. 35656

Parry E P 1963 J. Catal. 2371

Parker J G 1973 Appl. Opt. 122974

Peltinger V B, Schoppel H R and Gerischer H 1973 Ber. Bunsenges Phys. Chem. 77960

Peri J B 1965 J. Phys. Chem. 69220

Peterson R G and Powell R C 1978 Chem. Phys. Lett. 53366

Pichon C, Leliboux M, Fournier D and Boccara A C 1979 Appl. Phys. Lett. 351435

Poulet P, Chambron J and Unterreiner R 1980 J. Appl. Phys. 511738

Powell R C, Neikirk D P, Flaherty J M and Gualiteri J G 1980 J. Phys. Chem. Solids 41 345

Quimby R S and Yen W M 1979a Appl. Phys. Lett. 3543

Quimby R S and Yen W M 1979b Photoacoustic Spectroscopy Meeting Ames, Iowa, 1979, referred to by Crowley et al 1980

Quimby R S and Yen W M 1980a J. Appl. Phys. 514985

Quimby R S and Yen W M 1980b J. Appl. Phys. 511780

Razumova T K and Strabogatov I O 1977 Opt. Spectrosc. 42274

Read A W 1967 Adv. Mol. Relaxation Processes 1257

Roark J C, Palmer R A and Hutchinson J S 1979 Chem. Phys. Lett. 60112

Rockley M G 1979 Chem. Phys. Lett. 68455

Rockley M G and Waugh K M 1978 Chem. Phys. Lett. 54597

Rosencwaig A 1973 Opt. Commun. 7308

Rosencwaig A 1975 Anal. Chem. 47 592A

Rosencwaig A 1977 Rev. Sci. Instr. 481133

Rosencwaig A 1978a J. Appl. Phys. 492905

Rosencwaig A 1978b Adv. Electron. Electron. Phys. 46207

Rosencwaig A $1980 \mathrm{~J}$. Appl. Phys. 512210

Rosencwaig A and Busse G 1980 Appl. Phys. Lett. 36725

Rosencwaig A and Gersho A 1976 J. Appl. Phys. 4764

Sam C L and Shand M L 1979 Opt. Commun. 31174

Sequiera M A A, Ghizoni C C, Vargas J I, Menezes E A, Vargas H and Miranda L C M 1980 J. Appl. Phys. 511403

Slobodoskaya P V 1948 Izv. Akad. Nauk. SSSR Fiz. 12656

Starobogatov I O 1977 Opt. Spectrosc. 42274

Starobogatov I O 1979 Opt. Spectrosc. 46816

Tam A C and Patel C K N 1979a Appl. Phys. Lett. 34467

Tam A C and Patel C K N 1979b Appl. Phys. Lett. 35843

Viengerav M L 1938 Dokl. Akad. Nauk, SSSR 19687

Viengerov M L 1940 Izv. Akad. Nauk. SSSR Fiz. 54779

Wetsel Jr, G C and McDonald F A 1977 Appl. Phys. Lett. 30252

White R M 1962 IRE Trans. Instr. I-II 294

White R M 1963a J. Appl. Phys. 342123

White R M 1963b J. Appl. Phys. 343559

Wickramasinghe H K, Bray R C, Jipson V, Quate C F and Salcedo J R 1978 Appl, Phys. Lett. 33923

Wong K Y 1978 J. Appl. Phys. 493033

Wong K Y 1980 Aust. J. Phys. 3391

Wong Y H, Thomas R L and Hawkins G F 1978 Appl. Phys. Lett. 32538

Wong Y H, Thomas R L and Pouch J J 1979 Appl. Phys. Lett. 35368 